Key Words:

Rheology, CST, MST, SCIX

Retention:

Permanent

\title{
Rheology of Settled Solids in the Small Column Ion Exchange (SCIX) Process
}

\author{
Michael R. Poirier \\ Caitlin E. Ferguson \\ David C. Koopman \\ Tommy B. Edwards
}

June 20, 2011

Savannah River National Laboratory

Savannah River Nuclear Solutions

Aiken, SC 29808

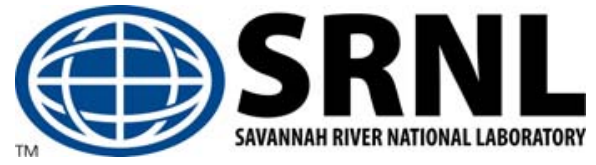




\section{DISCLAIMER}

This work was prepared under an agreement with and funded by the U.S. Government. Neither the U. S. Government or its employees, nor any of its contractors, subcontractors or their employees, makes any express or implied:

1. warranty or assumes any legal liability for the accuracy, completeness, or for the use or results of such use of any information, product, or process disclosed; or

2. representation that such use or results of such use would not infringe privately owned rights; or

3. endorsement or recommendation of any specifically identified commercial product, process, or service.

Any views and opinions of authors expressed in this work do not necessarily state or reflect those of the United States Government, or its contractors, or subcontractors.

Printed in the United States of America

Prepared for

U.S. Department of Energy 
Key Words:

Rheology, CST, MST, SCIX

Retention:

Permanent

\title{
Rheology of Settled Solids in the Small Column Ion Exchange (SCIX) Process
}

\author{
Michael R. Poirier \\ Caitlin E. Ferguson \\ David C. Koopman \\ Tommy B. Edwards
}

June 20, 2011

Savannah River National Laboratory

Savannah River Nuclear Solutions

Savannah River Site

Aiken, SC 29808 


\section{REVIEWS AND APPROVALS}

\section{Authors}

M. R. Poirier, SRNL, Advanced Characterization \& Processing

Date

C. E. Ferguson, SRNL, Advanced Characterization \& Processing

Date

D. C. Koopman, SRNL, Process Technology Programs

Date

T. B. Edwards, SRNL, Applied Computational Engineering and Statistics

Date

\section{Design Check}

C. A. Nash, SRNL, Advanced Characterization \& Processing

Date

\section{Management}

F. M. Pennebaker, Manager, SRNL, Advanced Characterization \& Processing $\quad$ Date

S. L. Marra, Manager, SRNL E\&CPT Research Programs

Date

\section{Customer}

T. H. Huff, Manager, SRR, SCIX Design Authority Manager

Date 


\section{TABLE OF CONTENTS}

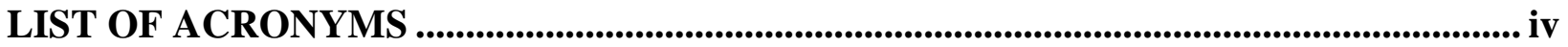

1.0 SUMMARY ….............................................................................................................................. 1

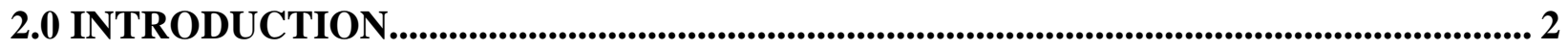

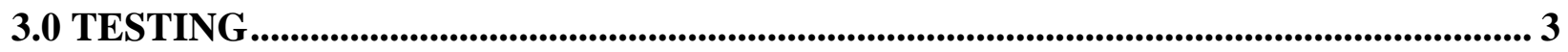

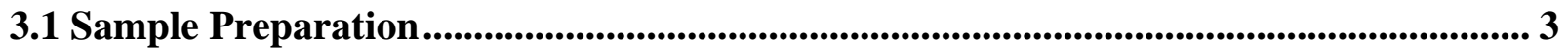

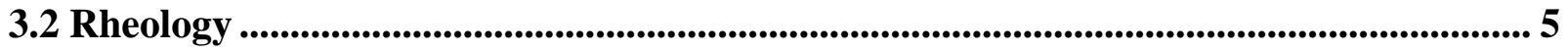

3.3 Statistical Design................................................................................................................... 7

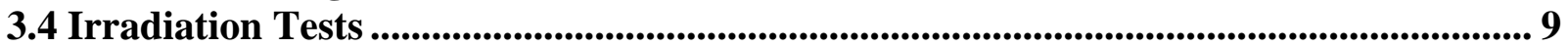

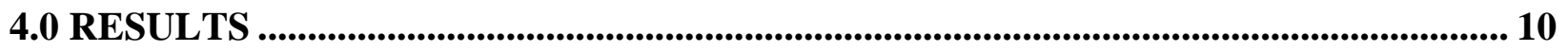

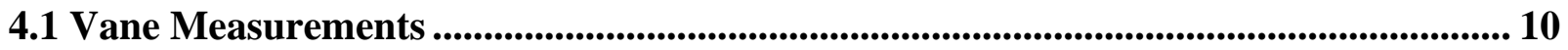

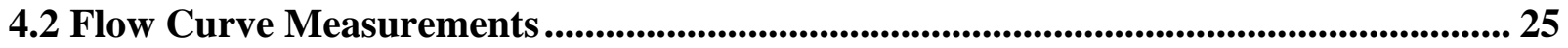

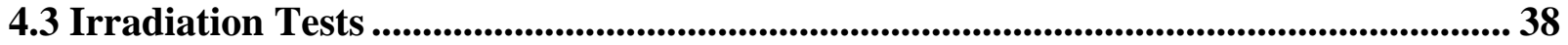

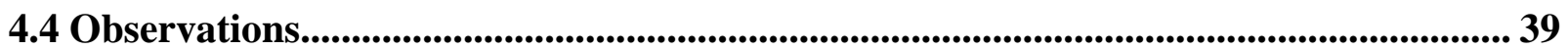

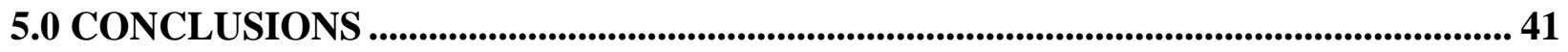

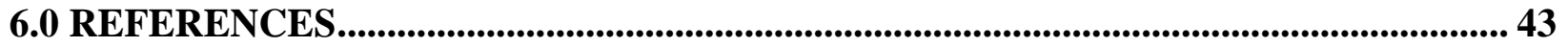

APPENDIX 1: Particle Size Distribution Curves ............................................................................. 44 


\section{LIST OF ACRONYMS}

$\begin{array}{ll}\text { ACTL } & \text { Aiken County Technology Laboratory } \\ \text { ARP } & \text { Actinide Removal Process } \\ \text { CST } & \text { Crystalline Silicotitanate } \\ \text { DI } & \text { De-ionized } \\ \text { DWPF } & \text { Defense Waste Processing Facility } \\ \text { MST } & \text { Monosodium Titanate } \\ \text { NIST } & \text { National Institute of Standards and Technology } \\ \text { ORNL } & \text { Oak Ridge National Laboratory } \\ \text { RMF } & \text { Rotary Microfilter } \\ \text { SCIX } & \text { Small Column Ion Exchange } \\ \text { SMP } & \text { Submersible Mixer Pump } \\ \text { SRNL } & \text { Savannah River National Laboratory } \\ \text { SRR } & \text { Savannah River Remediation } \\ \text { SRS } & \text { Savannah River Site } \\ \text { UOP } & \text { Universal Oil Products, LLC } \\ \text { VSL } & \text { Vitreous State Laboratory } \\ \text { XRD } & \text { X-ray Diffraction }\end{array}$




\subsection{SUMMARY}

The Small Column Ion Exchange (SCIX) process is being developed to remove cesium, strontium, and actinides from Savannah River Site (SRS) Liquid Waste using an existing waste tank as process housing. This method includes the addition of monosodium titanate (MST) to a waste tank containing salt solution and entrained sludge solids, followed by tank mixing and filtration. The filtrate is then processed through in-tank ion exchange columns containing crystalline silicotitanate (CST) media. While the process is operating, it is known that solid particles begin to settle in the tank and temperatures may reach beyond $45^{\circ} \mathrm{C}$. Previous testing has shown that sludge-MST slurries that sit for extended periods at elevated temperatures can develop large shear strengths, making them difficult to resuspend and remove from the tank.

The authors conducted rheological testing of mixtures containing various concentrations of sludge simulant, MST, and CST (three preparations) that were aged at different times (i.e., 0 to 13 weeks) and isothermally maintained to 30,45 , or $60^{\circ} \mathrm{C}$. Two types of grinding methodologies were employed to prepare CST for this testing, herein called Savannah River National Laboratory (SRNL) and Vitreous State Laboratory (VSL) ground materials. Unground CST particles were also tested. A small number of samples were irradiated prior to 4 week settling and $60^{\circ} \mathrm{C}$ temperature treatment, with exposures ranging from 0 to $100 \mathrm{MRad}$. Additional tests are also being conducted that will allow the solid particles to settle at $45^{\circ} \mathrm{C}$ for 6,12 , and 24 months.

The objectives of this task are to determine the impact of feed composition, settling time, and temperature on the shear strength, yield stress, and consistency of the slurries and to determine the impact of radiation on slurry rheology. The testing will determine the relative impact of these parameters rather than predict the shear strength, yield stress, and consistency as a function of feed and operating conditions. This document describes the rheology of slurries containing MST, CST, and simulated sludge that sat at indicated temperatures for up to 13 weeks. A previous SRNL report described preliminary rheology data of slurries containing MST and sludge. Preliminary results of the irradiation tests are also presented in this report, though additional data are still being collected. Rheology of the long term settling samples $(6,12$, and 24 months) and additional irradiation test results will be reported at a later date.

Conclusions from this analysis are as follows:

- Slurries containing MST and unground CST have the largest shear strength. Due to the high shear strengths measured in slurries containing unground CST, evaluations of specific tank contents and mixing capability should be performed prior to any addition of this material into a waste tank. Experimentally determined shear strengths indicate mixing could be problematic in mixtures containing unground CST.

- Increasing the ground CST fraction in the slurry increases the slurry shear strength, yield stress, and consistency.

- Increasing the sludge fraction in the slurry decreases the slurry shear strength, yield stress, and consistency.

- Slurries containing VSL ground CST have larger shear strength, yield stress, and consistency than slurries containing SRNL ground CST. 
- The effects of settling time and temperature on slurry shear strength are slurry dependent.

- No effects of settling time and temperature on slurry yield stress or consistency were observed.

- Radiation up to 100 MRad does not appear to affect properties of shear strength, yield stress, or consistency of process feeds.

\subsection{INTRODUCTION}

Savannah River Remediation (SRR) is working to deploy the SCIX process to remove cesium, strontium, and select actinides from SRS Liquid Waste using an existing waste tank as process housing. The process adds MST to the waste tank (i.e., Tank 41H) to sorb the strontium and actinides, removes the MST and entrained sludge with an in-riser rotary microfilter (RMF), and removes cesium with ion-exchange columns containing CST. The RMF returns the concentrated solids (i.e., MST and entrained sludge) to the waste tank. After being loaded with cesium, the CST is ground to reduce its size and transferred into a waste tank. The process baseline is to transfer the ground CST to Tank 40H, the Defense Waste Processing Facility (DWPF) feed tank. Efforts are underway to determine the feasibility of introducing the ground CST to Tank $41 \mathrm{H}$. The MST, sludge, and CST (if introduced to Tank $41 \mathrm{H}$ ) in the waste tank will be periodically transported to a sludge batch preparation tank (i.e., Tank $42 \mathrm{H}$ or Tank $51 \mathrm{H}$ ), and ultimately transported to DWPF.

Based on the SCIX flowsheet and the historical SRS baseline for entrained sludge composition of SRS salt solution $(600 \mathrm{mg} / \mathrm{L})$, the minimum ratio of MST to CST in Tank $41 \mathrm{H}$ will be 1:17. The minimum ratio of MST to entrained sludge will be 1:1.5. Initially, these ratios will be much larger; qualification of feed to the Actinide Removal Process (ARP) has shown the MST to sludge ratio to be much greater than 1:1.5. The maximum ratio of MST to CST and the maximum ratio of MST to sludge will be bounded by the tests containing only MST. In a salt tank (i.e., Tank 41H), the estimated ratio of MST to CST to sludge will be 1:17:1.5. In a sludge tank, the estimated ratio of MST to CST to sludge will be 1:17:660 ${ }^{1}$.

Previous SRNL and Oak Ridge National Laboratory (ORNL) testing shows that allowing slurries containing simulated sludge and MST to sit for extended times at elevated temperature leads to large increases in the slurry shear strength. ${ }^{2,3}$ In the SRNL tests, the sludge and MST settled for $1,14,28$, and 42 days at 25,35 , and $50^{\circ} \mathrm{C}$. In the ORNL tests, the sludge and MST settled for 0 to 61 days at 23 and $80^{\circ} \mathrm{C}$.

To assist SRR in designing the SCIX process, SRNL is conducting bench-scale rheology tests to determine the impact of changes in solids composition and process conditions on SCIX function. Testing was completed on representative mixtures of sludge simulant, MST, and CST (ground and unground) aged for different times (i.e., 0 to 13 weeks) at 30,45 , and $60^{\circ} \mathrm{C}^{1}$ A small number of samples were also irradiated (10 and $100 \mathrm{MRad})$ to monitor for resultant effects on slurry rheology. A previous SRNL report described preliminary rheology data from slurries containing MST or MST plus sludge. ${ }^{4}$ Additional samples have been prepared to examine the impact of long term settling (up to 24 months). Results of these long term settling tests will be documented in future reports. 
The objectives of this task are to determine which slurries have the largest shear strength, yield stress, and consistency ${ }^{\mathrm{a}}$, to determine the impact of settling time and temperature on the shear strength, yield stress, and consistency, and to determine the impact of radiation on slurry rheology. The shear strength data will provide an indication of the relative ease with which slurries can be suspended in a waste tank. The yield stress and consistency data will provide an indication of the relative ease with which slurries can be transferred between processes in the SRS Tank Farm.

\subsection{TESTING}

Evaluation of the rheological properties of samples included shear strength measurement of settled solids and flow curve measurement of slurries. Shear strength of settled solids was determined by vane measurement using a Haake RheoStress 600 rheometer. Flow curve measurements were taken using a Searle type bob/cup configuration and data were fit to a Bingham Plastic rheological model. All rheological measurements were obtained at the Aiken County Technology Laboratory (ACTL).

\subsection{SAMPLE PREPARATION}

The MST used for these experiments was selected to be representative of MST currently used at the ARP (Harrell Industries Lot\# 102209, pail 32 of 66 [feeds identified as L] and Blue Grass PO KK02101H, pail 19 of 24 [feeds identified as M]). Median particle size of this material was measured to average 16 micron. Prior to sample mixing, the MST was allowed to settle, and excess supernate was decanted. The material was mixed with an impeller to produce a homogeneous MST slurry of $22.3 \mathrm{wt} \%$ solids in $0.01 \mathrm{M} \mathrm{NaOH}$ supernate before being added to the samples.

Simulated Sludge Batch 6 was selected for use in these tests because it is slow-settling, viscous, and representative of sludge contained in Tanks $51 \mathrm{H}$ and $40 \mathrm{H}^{5}$. Because it is slow settling, it also mimics the physical properties of the entrained sludge expected in Tank $41 \mathrm{H}$. The simulated sludge slurry had an insoluble solids concentration of $11.35 \mathrm{wt} \%$, a soluble solids concentration of $6.3 \mathrm{wt} \%$ (primarily sodium nitrate, sodium nitrite, and sodium hydroxide), a median particle size of $\sim 23$ micron, a trimodal particle size distribution, and a yield stress of $50 \mathrm{~Pa}$ at $11.35 \mathrm{wt} \%$ insoluble solids. No settling or decanting was performed on this material. The sludge was mixed to produce a homogeneous slurry before being added to the samples.

The CST chosen for this work, IE-911, was from Lot \#208000056 produced by Universal Oil Products, LLC (UOP). Median particle size was measured at $\sim 400$ micron as received from UOP. Three preparations of this material were utilized for this work, designated as unground, VSL ground, and SRNL ground CSTs. ${ }^{\text {b }}$ Unground CST was not subjected to additional

\footnotetext{
${ }^{a}$ The term shear strength is sometimes referred to as a vane yield stress, settled solids yield stress, or vane shear strength. The term yield stress is sometimes referred to as a flow curve yield stress. The term consistency is sometimes referred to as a flow curve consistency, plastic viscosity, or infinite viscosity.

${ }^{\mathrm{b}}$ The method of CST grinding for any given sample is indicated throughout the text. The CST referred to as 'VSL ground' was ground by Hockmeyer/VSL via the method indicated in the text; this material was stored for only a few weeks before utilization in rheology tests. The CST referred to as 'SRNL ground' was processed by SRNL as indicated, but was stored outside for several years before utilization in these tests.
} 
processing after receipt from UOP. A subsample of CST IE-911 was ground by the Vitreous State Laboratory (VSL) using a Hockmeyer immersion mill, the same grinding technology proposed for the SCIX process. The VSL ground CST slurry contained $20-40 \mathrm{wt} \%$ insoluble solids with a median particle size of 2.5 micron (measured by Microtrac). SRNL ground CST was processed by SRNL using rotors and stators, with grinding times of 5 to 24 hours. ${ }^{6}$ The SRNL ground CST was a 20 wt \% slurry in water and had a median particle size of $~ 1.5$ micron. Particle size distribution plots are included in Appendix 1 of this report. The method of CST grinding for any given sample is indicated in the text. VSL ground CST samples are designated by the "M" grouping and SRNL ground CST samples are designated by the "L" grouping.

The supernate used for these samples was simulated Tank 37H supernate. It had a sodium concentration of $6.44 \mathrm{M}$ and a free hydroxide concentration of $2.57 \mathrm{M}$.

Samples were prepared in 2.5-inch diameter by 4-inch tall snap lid poly bottles (see Figure 1). The MST, CST, and sludge simulant were added to the bottles in ratios by weight to produce a nominal volume of $140 \mathrm{~mL}^{\mathrm{c}}$. Seventy $\mathrm{mL}$ of simulated Tank $37 \mathrm{H}$ salt solution $(6.44 \mathrm{M}$ sodium, 2.57 $\mathrm{M}$ free hydroxide, $0.35 \mathrm{M}$ aluminate, and $0.004 \mathrm{M}$ silicate) was added to each of the bottles. Bottle contents were mixed by hand to homogeneity, and then placed into ovens at 30, 45, or $60^{\circ} \mathrm{C}$ for the desired duration. Table 1 and Table 2 in Section 3.3 detail the relative amounts (by mass) of MST, CST, and simulated sludge added to the samples, as well as temperature and settling time.

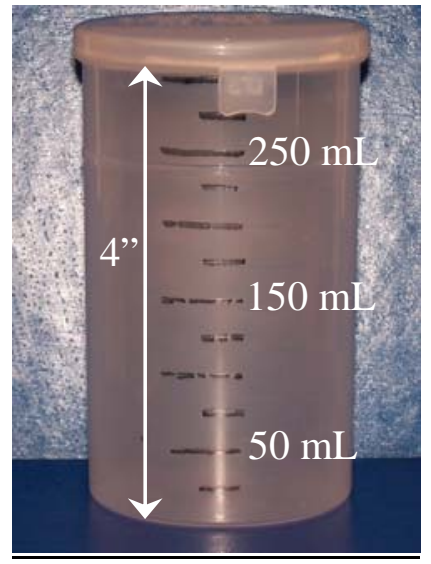

Figure 1. $300-\mathrm{mL}$ poly bottle with $25-\mathrm{mL}$ graduations

Samples were rotated to various quadrants within the oven on a weekly basis, to ensure equivalent heat treatment of all samples. Samples were visually inspected daily; any water loss caused by warping of the container lid was noted and de-ionized (DI) water added to original levels to compensate. Photographs of samples were taken before introduction to the oven and upon removal, to allow for pre- and post-treatment visual comparison.

Samples were removed from the ovens after allotted settling times, packed to minimize disturbance, and moved to ACTL for the characterization work. It was understood that small

\footnotetext{
c Samples containing MST and sludge in a 1:1.5 ratio are referred to as "MST plus low sludge". Samples containing MST and sludge in a 1:660 ratio are referred to as "MST plus high sludge".
} 
disturbances during shipping might impact the less densely packed settled solids, and that the primary focus of the study was on identifying samples which had undergone a significant transformation into a more compacted and difficult to suspend slurry rather than on identifying subtle differences between the various sample matrices. Settled solids volumes generally exceeded $125 \mathrm{~mL}$. In addition, small differences in water loss from evaporation may have had an impact on the flow curve rheological results. The technical literature shows yield stress to be an exponential function of insoluble solids. ${ }^{7}$ Therefore, small changes in insoluble solids could lead to large changes in yield stress of the test samples.

\subsection{RHEOLOGY}

Rheological measurements of yield strengths and flow curves were performed using the Haake RheoStress 600 rheometer at ACTL. Yield strength was measured with the FL22 vane sensor, shown in Figure 2. The four vane section has an effective diameter in rotation of $22 \mathrm{~mm}$; the vane was lowered into the sample matrix to a nominal vane depth of 15.5-31 mm into the settled solids (top to bottom of vane). In most samples, the bottom of the vane sensor measured $>15.5$ $\mathrm{mm}$ above the bottom of the settled solid layer (bottom of the snap lid jar). The positioned vane was rotated at 0.30 revolutions per minute for exactly 90 seconds, or $45 \%$ of a full single revolution. Solids in the spaces between the four vanes are forced to move relative to the solids above, below, and further out from the tips of the vanes. The friction between the moving and stationary particles creates torque, which is recorded by the rheometer. The maximum torque is typically observed in the first 15 seconds. This value is combined with the effective shearing area of the vanes to calculate the yield strength of the settled solids in Pascals (force/unit area). An example of vane data is given in Figure 3.

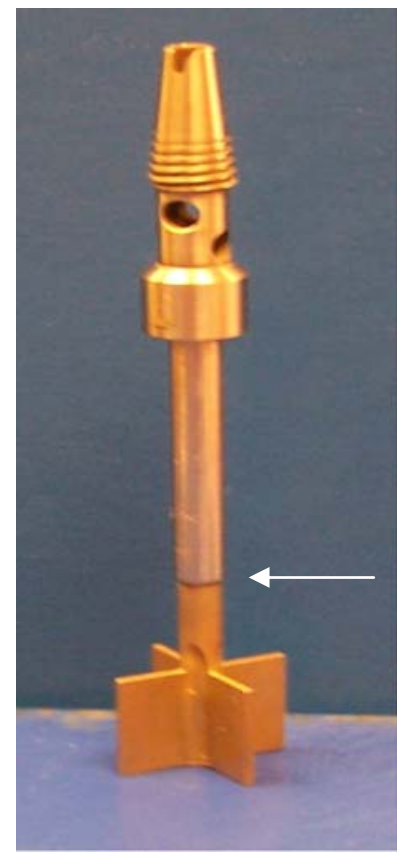

Figure 2. FL22 vane rotor used for yield strength measurements. Solids interface is indicated by the white arrow. 


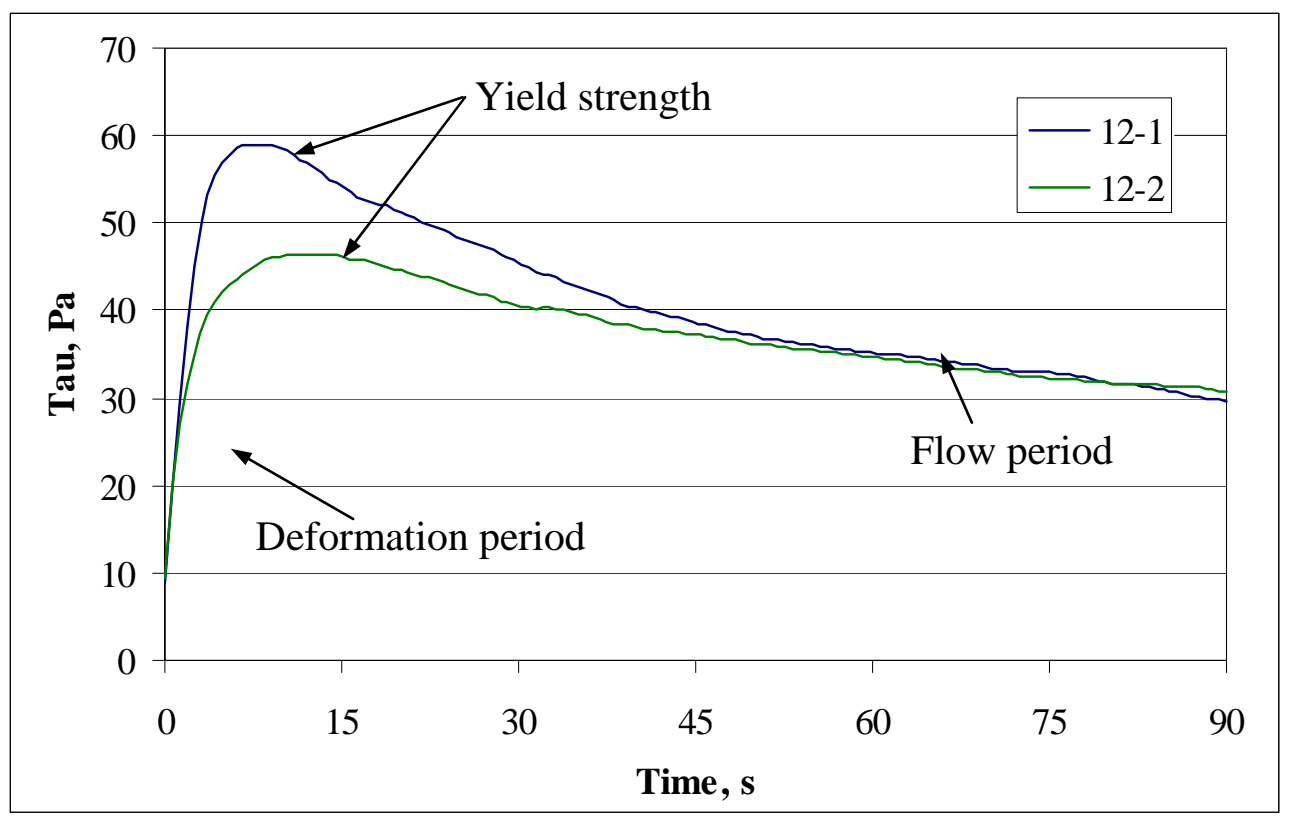

Figure 3. Example of vane data for sample 12

A vane measurement disturbs the settled solids such that the measurement can not be repeated in a vessel as small as the $300-\mathrm{mL}$ sample bottles used. Therefore, each sample was provided in duplicate so that two values of the shear strength could be obtained. Nevertheless, the measurement is delicate and the act of mounting the sample under the vane can cause some disturbance to the settled solids resulting in loss of reproducibility. It was also noted during this study that the replicate samples were not always identical with respect to settled solids volume, even though they presumably contained nearly identical masses of the matrix components. The majority of sample pairs with this feature had higher yield strength on the more compacted bed of solids, as would be intuitively expected. Vane measurements were performed at room temperature. A few samples were still warm when measured, but this was not believed to impact the results significantly.

The flow curve measurements provide rheological properties of mixed slurries and are applicable to the transport of slurries between facilities. Flow curve measurements were made using previously documented methods and the standard DWPF slurry sample protocols. ${ }^{8}$ Performance of the RS600 was checked regularly using National Institute of Standards and Technology (NIST) traceable viscosity standards. Flow curve measurements were made using a $25^{\circ} \mathrm{C}$ constant temperature bath surrounding the measurement cup.

Each sample in the L series was either diluted with a salt solution provided with the samples (one solution for all the diluted samples) or decanted to remove $25-\mathrm{mL}$ in order to obtain a second wt \% insoluble solids data point. The provided salt solution was measured to be approximately 29.9 wt \% dissolved solids by drying the sample with a halogen lamp. The three sludge-rich types, L5, L6, and L7 were decanted, while the other types were diluted. Decanting required centrifuging, since the remixed solids of the three sludge-rich types did not form sufficient supernate in a practical time span for any significant decanting. The samples in the $\mathrm{M}$ series were not diluted or decanted. 


\subsection{STATISTICAL DESIGN}

These tests examined the effect of temperature, settling time, and different combinations of MST, CST, and sludge solids on the rheological properties of the settled solids. Due to the large number of parameters requiring investigation, a statistical design of the experimental conditions was created to reduce the number of samples needed and to examine parameter interactions. Table 1 and Table 2 show the combination of treatments applied to the respective sludge simulant, CST, and MST solid mixtures. The resulting rheology data are fitted to a polynomial function such as the one listed in equation 1, where $\mathrm{Y}$ is the slurry shear strength, yield stress, or consistency. In addition, statistical analyses were performed to determine which parameters affect the shear strength, yield stress, and consistency. The term "feed slurry factor" is included in equation [1] to account for differences in rheological properties resulting from the different feed material. The purpose of equation [1] is to try to determine the impact of parameters, such as feed, time, and temperature, on the slurry rheological properties, not to develop a predictive model equation.

$$
\mathrm{Y}=\mathrm{a}+\mathrm{b} \text { (feed slurry factor) }+\mathrm{c} \text { (time factor) }+\mathrm{d} \text { (temperature factor) }
$$

Table 1. Rheology Test Conditions with SRNL Ground CST Content

\begin{tabular}{|c|c|c|c|c|}
\hline Sample \# & Feed & Feed Composition & $\begin{array}{c}\text { Time } \\
\text { (weeks) }\end{array}$ & Temp ( ${ }^{\circ}$ C) \\
\hline 1 & L4 & MST, low sludge & 1 & 60 \\
\hline 2 & L7 & MST, high sludge & 13 & 60 \\
\hline 3 & L4 & MST, low sludge & 13 & 60 \\
\hline 4 & L6 & MST, unground CST, high sludge & 1 & 60 \\
\hline 4B & L5 & MST, ground CST, high sludge & 1 & 6 \\
\hline 5 & L2 & MST, ground CST & 13 & 60 \\
\hline 6 & L1 & MST & 1 & 60 \\
\hline 7 & L9 & MST, unground CST, low sludge & 4 & 60 \\
\hline $7 B$ & L8 & MST, ground CST, low sludge & 4 & 60 \\
\hline 8 & L6 & MST, unground CST, high sludge & 10 & 60 \\
\hline 9 & L3 & MST, unground CST & 1 & 60 \\
\hline 10 & L3 & MST, unground CST & 13 & 60 \\
\hline 11 & L4 & MST, low sludge & 13 & 30 \\
\hline 12 & L7 & MST, high sludge & 1 & 30 \\
\hline 13 & L1 & MST & 1 & 30 \\
\hline 14 & L3 & MST, unground CST & 1 & 30 \\
\hline $14 B$ & L2 & MST, ground CST & 1 & 30 \\
\hline 15 & L5 & MST, ground CST, high sludge & 10 & 30 \\
\hline 16 & L3 & MST, unground CST & 4 & 30 \\
\hline 17 & L2 & MST, ground CST & 13 & 30 \\
\hline 18 & L6 & MST, unground CST, high sludge & 13 & 30 \\
\hline 19 & L9 & MST, unground CST, low sludge & 1 & 30 \\
\hline $19 B$ & L8 & MST, ground CST, low sludge & 1 & 30 \\
\hline 20 & L1 & MST & 13 & 30 \\
\hline 21 & L6 & MST, unground CST, high sludge & 1 & 30 \\
\hline 22 & L4 & MST, low sludge & 1 & 30 \\
\hline & & & & \\
\hline
\end{tabular}


SRNL-STI-2011-00311, REV.0

\begin{tabular}{|c|c|c|c|c|}
\hline Sample \# & Feed & Feed Composition & $\begin{array}{c}\text { Time } \\
\text { (weeks) }\end{array}$ & $\operatorname{Temp}\left({ }^{\circ} \mathrm{C}\right)$ \\
\hline 23 & L7 & MST, high sludge & 13 & 30 \\
\hline 24 & L5 & MST, ground CST, high sludge & 13 & 30 \\
\hline 25 & $\mathrm{~L} 2$ & MST, ground CST & 10 & 30 \\
\hline 26 & L3 & MST, unground CST & 1 & 30 \\
\hline 27 & L6 & MST, unground CST, high sludge & 1 & 30 \\
\hline $27 \mathrm{~B}$ & L5 & MST, ground CST, high sludge & 1 & 30 \\
\hline 28 & L8 & MST, ground CST, low sludge & 13 & 30 \\
\hline 29 & L1 & MST & 4 & 30 \\
\hline 30 & L3 & MST, unground CST & 13 & 30 \\
\hline 31 & L9 & MST, unground CST, low sludge & 1 & 60 \\
\hline $31 \mathrm{~B}$ & L8 & MST, ground CST, low sludge & 1 & 60 \\
\hline 32 & L6 & MST, unground CST, high sludge & 13 & 60 \\
\hline 33 & L3 & MST, unground CST & 4 & 60 \\
\hline 34 & L3 & MST, unground CST & 1 & 60 \\
\hline $34 \mathrm{~B}$ & L2 & MST, ground CST & 1 & 60 \\
\hline 35 & L7 & MST, high sludge & 1 & 60 \\
\hline 36 & L5 & MST, ground CST, high sludge & 13 & 60 \\
\hline 37 & L4 & MST, low sludge & 10 & 60 \\
\hline 38 & L8 & MST, ground CST, low sludge & 13 & 60 \\
\hline 39 & L1 & MST & 13 & 60 \\
\hline 40 & L6 & MST, unground CST, high sludge & 1 & 60 \\
\hline 41 & L7 & MST, high sludge & 4 & 45 \\
\hline 42 & L3 & MST, unground CST & 1 & 45 \\
\hline 42B & $\mathrm{L} 2$ & MST, ground CST & 1 & 45 \\
\hline 43 & L6 & MST, unground CST, high sludge & 4 & 45 \\
\hline 44 & L3 & MST, unground CST & 10 & 45 \\
\hline 45 & L6 & MST, unground CST, high sludge & 1 & 45 \\
\hline 45B & L5 & MST, ground CST, high sludge & 1 & 45 \\
\hline 46 & L7 & MST, high sludge & 13 & 45 \\
\hline 47 & L1 & MST & 13 & 45 \\
\hline 48 & L8 & MST, ground CST, low sludge & 10 & 45 \\
\hline 49 & L4 & MST, low sludge & 13 & 45 \\
\hline 50 & L4 & MST, low sludge & 1 & 45 \\
\hline 51 & L6 & MST, unground CST, high sludge & 8 & 60 \\
\hline 52 & L5 & MST, ground CST, high sludge & 8 & 30 \\
\hline 53 & L2 & MST, ground CST & 8 & 30 \\
\hline 54 & L4 & MST, low sludge & 8 & 60 \\
\hline 55 & L3 & MST, unground CST & 8 & 45 \\
\hline 56 & L9 & MST, unground CST, low sludge & 8 & 45 \\
\hline 57 & L1 & MST & 8 & 45 \\
\hline
\end{tabular}

Table 2. Rheology Test Conditions with VSL Ground CST Content

\begin{tabular}{|c|c|c|c|c|}
\hline Sample \# & Feed & Feed Composition & $\begin{array}{c}\text { Time } \\
\text { (weeks) }\end{array}$ & Temp $\left({ }^{\circ} \mathbf{C}\right)$ \\
\hline 101 & M2 & MST, ground CST & 1 & 30 \\
\hline 102 & M5 & MST, ground CST, high sludge & 1 & 30 \\
\hline 103 & M8 & MST, ground CST, low sludge & 1 & 30 \\
\hline 104 & M11 & MST, ground CST, med. sludge & 1 & 30 \\
\hline
\end{tabular}


SRNL-STI-2011-00311, REV.0

\begin{tabular}{|c|c|c|c|c|}
\hline Sample \# & Feed & Feed Composition & $\begin{array}{c}\text { Time } \\
\text { (weeks) }\end{array}$ & $\operatorname{Temp}\left({ }^{\circ} \mathrm{C}\right)$ \\
\hline 105 & M2 & MST, ground CST & 4 & 30 \\
\hline 106 & M5 & MST, ground CST, high sludge & 4 & 30 \\
\hline 107 & M8 & MST, ground CST, low sludge & 4 & 30 \\
\hline 108 & M11 & MST, ground CST, med. sludge & 4 & 30 \\
\hline 109 & M2 & MST, ground CST & 13 & 30 \\
\hline 110 & M5 & MST, ground CST, high sludge & 13 & 30 \\
\hline 111 & M8 & MST, ground CST, low sludge & 13 & 30 \\
\hline 112 & M2 & MST, ground CST & 10 & 30 \\
\hline 113 & M5 & MST, ground CST, high sludge & 10 & 30 \\
\hline 114 & M8 & MST, ground CST, low sludge & 10 & 30 \\
\hline 115 & M2 & MST, ground CST & 4 & 45 \\
\hline 116 & M5 & MST, ground CST, high sludge & 4 & 45 \\
\hline 117 & M8 & MST, ground CST, low sludge & 4 & 45 \\
\hline 118 & M11 & MST, ground CST, med. sludge & 4 & 45 \\
\hline 119 & M1 & MST & 8 & 45 \\
\hline 120 & M2 & MST, ground CST & 1 & 45 \\
\hline 121 & M5 & MST, ground CST, high sludge & 1 & 45 \\
\hline 122 & M8 & MST, ground CST, low sludge & 1 & 45 \\
\hline 123 & M2 & MST, ground CST & 13 & 45 \\
\hline 124 & M5 & MST, ground CST, high sludge & 13 & 45 \\
\hline 125 & M8 & MST, ground CST, low sludge & 13 & 45 \\
\hline 126 & M2 & MST, ground CST & 10 & 45 \\
\hline 127 & M5 & MST, ground CST, high sludge & 10 & 45 \\
\hline 128 & M8 & MST, ground CST, low sludge & 10 & 45 \\
\hline 129 & M2 & MST, ground CST & 1 & 60 \\
\hline 130 & M5 & MST, ground CST, high sludge & 1 & 60 \\
\hline 131 & M8 & MST, ground CST, low sludge & 1 & 60 \\
\hline 132 & M11 & MST, ground CST, med. sludge & 1 & 60 \\
\hline 133 & M2 & MST, ground CST & 4 & 60 \\
\hline 134 & M5 & MST, ground CST, high sludge & 4 & 60 \\
\hline 135 & M8 & MST, ground CST, low sludge & 4 & 60 \\
\hline 136 & M11 & MST, ground CST, med. sludge & 4 & 60 \\
\hline 137 & M1 & MST & 4 & 60 \\
\hline 138 & M7 & MST, high sludge & 4 & 60 \\
\hline 139 & M2 & MST, ground CST & 13 & 60 \\
\hline 140 & M5 & MST, ground CST, high sludge & 13 & 60 \\
\hline 141 & M8 & MST, ground CST, low sludge & 13 & 60 \\
\hline 142 & M7 & MST, high sludge & 13 & 60 \\
\hline 143 & M2 & MST, ground CST & 10 & 60 \\
\hline 144 & M5 & MST, ground CST, high sludge & 10 & 60 \\
\hline 145 & M8 & MST, ground CST, low sludge & 10 & 60 \\
\hline 146 & M7 & MST, high sludge & 10 & 60 \\
\hline 147 & M10 & MST, ground CST (1:1) & 4 & 45 \\
\hline 148 & M12 & MST, ground CST, sludge (1:1:1) & 4 & 45 \\
\hline
\end{tabular}

\subsection{IRRADIATION TESTS}

Three $60 \mathrm{~mL}$ aliquots of each of the sample mixtures in Table 3 were prepared for irradiation, comprised of $40 \mathrm{~mL}$ of solids and $20 \mathrm{~mL}$ of Tank $37 \mathrm{H}$ salt solution. Materials were hand mixed and placed into stainless steel vessels for irradiation to the specified dose (0,10, or $100 \mathrm{MRad})$. 
Irradiations were completed using a JL Shepard Model 109 Cobalt-60 irradiator in the SRNL Gamma Irradiation Facility. Dose rates at the time of exposure were on the order of 5E3 Rad per hour, and temperature within the irradiator was nominally $25^{\circ} \mathrm{C} \pm 5$. Following irradiation, the like samples were removed from the stainless steel vessels, blended, and realiquoted into poly snap lid containers to produce duplicate samples of $\sim 60 \mathrm{~mL}$ of solids and $\sim 30 \mathrm{~mL}$ of supernate. Samples were placed in an oven at $60^{\circ} \mathrm{C}$ for four weeks. Following the settling, the rheological properties were measured.

Table 3. Tests to determine if radiation affects rheological data.

\begin{tabular}{|c|c|c|c|c|}
\hline Sample \# & Temp $\left({ }^{\circ} \mathbf{C}\right)$ & Time (weeks) & Feed & Dose (MRad) \\
\hline 1 & 60 & 4 & M8 & 0 \\
\hline 2 & 60 & 4 & M5 & 10 \\
\hline 3 & 60 & 4 & M8 & 10 \\
\hline 4 & 60 & 4 & M5 & 100 \\
\hline 5 & 60 & 4 & M8 & M5 \\
\hline 6 & 60 & 4 & M5 MST + Sludge + VSL ground CST (1:660:17 wt ratio) \\
\hline \multicolumn{5}{c}{ M8 = MST + Sludge + VSL ground CST (1:1.5:17 ratio) } \\
\hline
\end{tabular}

\subsection{RESULTS}

\subsection{VANE MEASUREMENTS}

Table 4 summarizes the measured shear strength as a function of feed and settling conditions. Replicate samples, in general, showed good agreement. Table 5 shows the average measured shear strength as a function of feed slurry. The highest shear strength samples appear to be those containing MST and unground CST (L3), followed by samples containing MST only (L1), MST plus VSL ground CST (M2), and samples containing MST, unground CST, and sludge in a 1:17:1.5 ratio (L9). The average shear strength of the MST plus unground CST samples is 2019 $\mathrm{Pa}$. The average shear strength of the MST only samples is $765 \mathrm{~Pa}$ (batch L) and $172 \mathrm{~Pa}$ (batch $\mathrm{M})$. The average shear strength of the MST plus VSL ground CST samples is $749 \mathrm{~Pa}$. The average shear strength of the samples containing MST, unground CST, and sludge in a 1:17:1.5 ratio is $458 \mathrm{~Pa}$.

Table 4. Measured Slurry Rheological Properties

\begin{tabular}{|c|c|c|c|c|c|c|}
\hline Feed & Feed Materials & $\begin{array}{l}\text { Temp } \\
\left({ }^{\circ} \mathrm{C}\right)\end{array}$ & $\begin{array}{c}\text { Time } \\
\text { (weeks) }\end{array}$ & $\begin{array}{c}\text { Shear Strength } \\
(\mathbf{P a})\end{array}$ & $\begin{array}{c}\text { Yield Stress } \\
(\mathbf{P a})\end{array}$ & $\begin{array}{c}\text { Consistency } \\
\text { (cP) }\end{array}$ \\
\hline L1 & MST & 30 & 1 & 199 & 5 & 16 \\
\hline L1 & MST & 30 & 1 & 236 & 10.3 & 24 \\
\hline L1 & MST & 60 & 1 & 106 & 5.3 & 14 \\
\hline L1 & MST & 60 & 1 & 119 & 10 & 24 \\
\hline L1 & MST & 30 & 4 & 489 & 7.5 & 18 \\
\hline L1 & MST & 30 & 4 & 533 & 11.3 & 27 \\
\hline L1 & MST & 45 & 8 & $3592^{d}$ & 12.4 & 26 \\
\hline L1 & MST & 45 & 8 & $2036^{\mathrm{d}}$ & 11.8 & 25 \\
\hline L1 & MST & 30 & 13 & 484 & 8.6 & 19 \\
\hline L1 & MST & 30 & 13 & 352 & 8.7 & 19 \\
\hline L1 & MST & 45 & 13 & 458 & 12 & 31 \\
\hline
\end{tabular}


SRNL-STI-2011-00311, REV.0

\begin{tabular}{|c|c|c|c|c|c|c|}
\hline Feed & Feed Materials & $\begin{array}{l}\text { Temp } \\
\left({ }^{\circ} \mathrm{C}\right)\end{array}$ & $\begin{array}{c}\text { Time } \\
\text { (weeks) }\end{array}$ & $\begin{array}{c}\text { Shear Strength } \\
\text { (Pa) }\end{array}$ & $\begin{array}{c}\text { Yield Stress } \\
(\mathbf{P a})\end{array}$ & $\begin{array}{c}\text { Consistency } \\
\text { (cP) }\end{array}$ \\
\hline L1 & MST & 45 & 13 & 421 & 7.8 & 21 \\
\hline L1 & MST & 60 & 13 & 762 & 37 & 115 \\
\hline L1 & MST & 60 & 13 & 927 & 36 & 113 \\
\hline $\mathrm{L} 2$ & MST, SRNL ground CST & 30 & 1 & 52 & 29 & 21 \\
\hline $\mathrm{L} 2$ & MST, SRNL ground CST & 30 & 1 & 49 & 28 & 21 \\
\hline $\mathrm{L} 2$ & MST, SRNL ground CST & 45 & 1 & 21 & 9.2 & 13 \\
\hline $\mathrm{L} 2$ & MST, SRNL ground CST & 45 & 1 & 25 & 8.8 & 13 \\
\hline $\mathrm{L} 2$ & MST, SRNL ground CST & 60 & 1 & 60 & 27 & 25 \\
\hline $\mathrm{L} 2$ & MST, SRNL ground CST & 60 & 1 & 34 & 9.3 & 14 \\
\hline L3 & MST, unground CST & 30 & 1 & 874 & 37 & 110 \\
\hline L3 & MST, unground CST & 30 & 1 & 888 & 75 & 108 \\
\hline L3 & MST, unground CST & 30 & 1 & 1725 & 91 & 285 \\
\hline L3 & MST, unground CST & 30 & 1 & 1541 & 76 & 235 \\
\hline L3 & MST, unground CST & 45 & 1 & 1528 & 151 & 454 \\
\hline L3 & MST, unground CST & 45 & 1 & 1167 & 96 & 245 \\
\hline L3 & MST, unground CST & 60 & 1 & 7679 & $\mathrm{n} / \mathrm{m}$ & $\mathrm{n} / \mathrm{m}$ \\
\hline L3 & MST, unground CST & 60 & 1 & 11271 & $\mathrm{n} / \mathrm{m}$ & $\mathrm{n} / \mathrm{m}$ \\
\hline L3 & MST, unground CST & 60 & 1 & 426 & 76 & 234 \\
\hline L3 & MST, unground CST & 60 & 1 & 1132 & 127 & 372 \\
\hline L3 & MST, unground CST & 30 & 4 & 1167 & 144 & 460 \\
\hline L3 & MST, unground CST & 30 & 4 & 1901 & 96 & 340 \\
\hline L3 & MST, unground CST & 60 & 4 & 1262 & 126 & 526 \\
\hline L3 & MST, unground CST & 60 & 4 & 1310 & 175 & 720 \\
\hline L3 & MST, unground CST & 30 & 8 & 745 & 37 & 103 \\
\hline L3 & MST, unground CST & 30 & 8 & 810 & 38 & 99 \\
\hline L3 & MST, unground CST & 45 & 8 & 795 & 88 & 310 \\
\hline L3 & MST, unground CST & 45 & 8 & 573 & 77 & 402 \\
\hline L3 & MST, unground CST & 45 & 10 & 707 & 83 & 290 \\
\hline L3 & MST, unground CST & 45 & 10 & 534 & 57 & 210 \\
\hline L3 & MST, unground CST & 30 & 13 & 733 & 80 & 330 \\
\hline L3 & MST, unground CST & 30 & 13 & 963 & 98 & 380 \\
\hline L3 & MST, unground CST & 60 & 13 & 4956 & $\mathrm{n} / \mathrm{m}$ & $\mathrm{n} / \mathrm{m}$ \\
\hline L3 & MST, unground CST & 60 & 13 & 3760 & $\mathrm{n} / \mathrm{m}$ & $\mathrm{n} / \mathrm{m}$ \\
\hline L4 & MST, low sludge & 30 & 1 & 32 & 2.7 & 7.3 \\
\hline L4 & MST, low sludge & 30 & 1 & 36 & 3.4 & 7.3 \\
\hline L4 & MST, low sludge & 45 & 1 & 19 & 1.85 & 6.4 \\
\hline L4 & MST, low sludge & 45 & 1 & 44 & 2.4 & 6.3 \\
\hline L4 & MST, low sludge & 60 & 1 & 38 & 2.1 & 6.5 \\
\hline L4 & MST, low sludge & 60 & 1 & 35 & 1.9 & 6.4 \\
\hline L4 & MST, low sludge & 60 & 8 & 86 & 1.8 & 6.2 \\
\hline L4 & MST, low sludge & 60 & 8 & 100 & 2.5 & 7.2 \\
\hline L4 & MST, low sludge & 60 & 10 & 77 & 3.3 & 7.7 \\
\hline L4 & MST, low sludge & 60 & 10 & 156 & 4.4 & 9.4 \\
\hline L4 & MST, low sludge & 30 & 13 & 35 & 3.2 & 7.7 \\
\hline L4 & MST, low sludge & 30 & 13 & 20 & 4 & 7.8 \\
\hline L4 & MST, low sludge & 45 & 13 & 53 & 3.5 & 7.2 \\
\hline
\end{tabular}


SRNL-STI-2011-00311, REV.0

\begin{tabular}{|c|c|c|c|c|c|c|}
\hline Feed & Feed Materials & $\begin{array}{l}\text { Temp } \\
\left({ }^{\circ} \mathrm{C}\right)\end{array}$ & $\begin{array}{c}\text { Time } \\
\text { (weeks) }\end{array}$ & $\begin{array}{c}\text { Shear Strength } \\
\text { (Pa) }\end{array}$ & $\begin{array}{l}\text { Yield Stress } \\
\text { (Pa) }\end{array}$ & $\begin{array}{c}\text { Consistency } \\
\text { (cP) }\end{array}$ \\
\hline L4 & MST, low sludge & 45 & 13 & 63 & 2.8 & 6.9 \\
\hline L4 & MST, low sludge & 60 & 13 & 118 & 3 & 7.5 \\
\hline L4 & $\begin{array}{l}\text { MST, low sludge } \\
\end{array}$ & 60 & 13 & 130 & 2.9 & 7.2 \\
\hline L5 & MST, SRNL ground CST, high sludge & 30 & 1 & 16 & 5.4 & 8.8 \\
\hline L5 & MST, SRNL ground CST, high sludge & 30 & 1 & 15 & 5.5 & 8.8 \\
\hline L5 & MST, SRNL ground CST, high sludge & 45 & 1 & 19 & 3.2 & 7.9 \\
\hline L5 & MST, SRNL ground CST, high sludge & 45 & 1 & 19 & 3.3 & 8.3 \\
\hline L5 & MST, SRNL ground CST, high sludge & 60 & 1 & 33 & 2.1 & 7.4 \\
\hline L5 & MST, SRNL ground CST, high sludge & 60 & 1 & 32 & 2.2 & 7.6 \\
\hline L6 & MST, unground CST, high sludge & 30 & 1 & 50 & 3.4 & 10.4 \\
\hline L6 & MST, unground CST, high sludge & 30 & 1 & 57 & 5.4 & 11.3 \\
\hline L6 & MST, unground CST, high sludge & 30 & 1 & 53 & 5.5 & 8 \\
\hline L6 & MST, unground CST, high sludge & 30 & 1 & 61 & 5.4 & 8 \\
\hline L6 & MST, unground CST, high sludge & 45 & 1 & 63 & 3 & 7.3 \\
\hline L6 & MST, unground CST, high sludge & 45 & 1 & 62 & 2.7 & 7.15 \\
\hline L6 & MST, unground CST, high sludge & 60 & 1 & 79 & 2 & 6.4 \\
\hline L6 & MST, unground CST, high sludge & 60 & 1 & 74 & 2.1 & 6.7 \\
\hline L6 & MST, unground CST, high sludge & 60 & 1 & 73 & 2 & 9.4 \\
\hline L6 & MST, unground CST, high sludge & 60 & 1 & 59 & 1.7 & 9.3 \\
\hline L6 & MST, unground CST, high sludge & 45 & 4 & 80 & 2.6 & 10 \\
\hline L6 & MST, unground CST, high sludge & 45 & 4 & 66 & 2.1 & 10.3 \\
\hline L6 & MST, unground CST, high sludge & 30 & 8 & 81 & $\mathrm{n} / \mathrm{m}$ & $\mathrm{n} / \mathrm{m}$ \\
\hline L6 & MST, unground CST, high sludge & 30 & 8 & 69 & $\mathrm{n} / \mathrm{m}$ & $\mathrm{n} / \mathrm{m}$ \\
\hline L6 & MST, unground CST, high sludge & 60 & 8 & 107 & 4.9 & 15 \\
\hline L6 & MST, unground CST, high sludge & 60 & 8 & 150 & 2 & 9.9 \\
\hline L6 & MST, unground CST, high sludge & 60 & 10 & 199 & 3.6 & 11 \\
\hline L6 & MST, unground CST, high sludge & 60 & 10 & 196 & 3.9 & 11.3 \\
\hline L6 & MST, unground CST, high sludge & 30 & 13 & 78 & 4 & 10.1 \\
\hline L6 & MST, unground CST, high sludge & 30 & 13 & 71 & 4.8 & 10.6 \\
\hline L6 & MST, unground CST, high sludge & 30 & 13 & 89 & $\mathrm{n} / \mathrm{m}$ & $\mathrm{n} / \mathrm{m}$ \\
\hline L6 & MST, unground CST, high sludge & 30 & 13 & 78 & $\mathrm{n} / \mathrm{m}$ & $\mathrm{n} / \mathrm{m}$ \\
\hline L6 & MST, unground CST, high sludge & 60 & 13 & 184 & 6.7 & 14.7 \\
\hline L6 & MST, unground CST, high sludge & 60 & 13 & 233 & 9.9 & 17 \\
\hline L6 & MST, unground CST, high sludge & 60 & 13 & 157 & $\mathrm{n} / \mathrm{m}$ & $\mathrm{n} / \mathrm{m}$ \\
\hline L6 & MST, unground CST, high sludge & 60 & 13 & 141 & $\mathrm{n} / \mathrm{m}$ & $\mathrm{n} / \mathrm{m}$ \\
\hline L7 & MST, high sludge & 30 & 1 & 59 & 5.1 & 12.9 \\
\hline L7 & MST, high sludge & 30 & 1 & 47 & 5.1 & 12.9 \\
\hline L7 & MST, high sludge & 60 & 1 & 79 & 2 & 6.7 \\
\hline L7 & MST, high sludge & 60 & 1 & 68 & 1.9 & 6.5 \\
\hline L7 & MST, high sludge & 45 & 4 & 74 & 2.63 & 7.39 \\
\hline L7 & MST, high sludge & 45 & 4 & 95 & 2.75 & 7.36 \\
\hline L7 & MST, high sludge & 30 & 13 & 71 & 2.4 & 6.6 \\
\hline L7 & MST, high sludge & 30 & 13 & 80 & 4.5 & 8.1 \\
\hline L7 & MST, high sludge & 45 & 13 & 75 & 3.5 & 7.4 \\
\hline L7 & MST, high sludge & 45 & 13 & 90 & 3.4 & 7.3 \\
\hline L7 & MST, high sludge & 60 & 13 & 601 & 3.7 & 7.9 \\
\hline
\end{tabular}


SRNL-STI-2011-00311, REV.0

\begin{tabular}{|c|c|c|c|c|c|c|}
\hline Feed & Feed Materials & $\begin{array}{l}\text { Temp } \\
\left({ }^{\circ} \mathrm{C}\right)\end{array}$ & $\begin{array}{c}\text { Time } \\
\text { (weeks) }\end{array}$ & $\begin{array}{c}\text { Shear Strength } \\
\text { (Pa) }\end{array}$ & $\begin{array}{c}\text { Yield Stress } \\
(\mathbf{P a})\end{array}$ & $\begin{array}{c}\text { Consistency } \\
\text { (cP) }\end{array}$ \\
\hline L8 & MST, SRNL ground CST, low sludge & 30 & 1 & 24 & 10 & 15 \\
\hline L8 & MST, SRNL ground CST, low sludge & 30 & 1 & 26 & 10 & 15 \\
\hline L8 & MST, SRNL ground CST, low sludge & 60 & 1 & 37 & 8.9 & 15 \\
\hline L8 & MST, SRNL ground CST, low sludge & 60 & 1 & 42 & 10.1 & 16 \\
\hline L8 & MST, SRNL ground CST, low sludge & 60 & 4 & 89 & 11.8 & 19 \\
\hline L8 & MST, SRNL ground CST, low sludge & 60 & 4 & 46 & 9.7 & 16 \\
\hline L9 & MST, unground CST, low sludge & 30 & 1 & 19 & 18 & 32 \\
\hline L9 & MST, unground CST, low sludge & 30 & 1 & 111 & 27 & 41 \\
\hline L9 & MST, unground CST, low sludge & 60 & 1 & 409 & 15 & 31 \\
\hline L9 & MST, unground CST, low sludge & 60 & 1 & 954 & 20 & 45 \\
\hline L9 & MST, unground CST, low sludge & 60 & 4 & 276 & 34 & 72 \\
\hline L9 & MST, unground CST, low sludge & 60 & 4 & 468 & 16 & 33 \\
\hline L9 & MST, unground CST, low sludge & 45 & 8 & 60 & 33 & 40 \\
\hline L9 & MST, unground CST, low sludge & 45 & 8 & 121 & 35 & 37 \\
\hline L9 & MST, unground CST, low sludge & 30 & 13 & 101 & $\mathrm{n} / \mathrm{m}$ & $\mathrm{n} / \mathrm{m}$ \\
\hline L9 & MST, unground CST, low sludge & 30 & 13 & 1166 & $\mathrm{n} / \mathrm{m}$ & $\mathrm{n} / \mathrm{m}$ \\
\hline L9 & MST, unground CST, low sludge & 60 & 13 & 1355 & $\mathrm{n} / \mathrm{m}$ & $\mathrm{n} / \mathrm{m}$ \\
\hline M1 & MST & 45 & 4 & 105 & 1.4 & 7.6 \\
\hline M1 & MST & 45 & 4 & 118 & $\mathrm{n} / \mathrm{m}$ & $\mathrm{n} / \mathrm{m}$ \\
\hline M1 & MST & 60 & 4 & 129 & 1.6 & 8.5 \\
\hline M1 & MST & 60 & 4 & 135 & $\mathrm{n} / \mathrm{m}$ & $\mathrm{n} / \mathrm{m}$ \\
\hline M1 & MST & 45 & 8 & 351 & 1.9 & 9.5 \\
\hline M1 & MST & 45 & 8 & 194 & 2.7 & 13.3 \\
\hline M11 & MST, VSL ground CST, medium sludge & 30 & 1 & 12 & 8.1 & 10.2 \\
\hline M11 & MST, VSL ground CST, medium sludge & 30 & 1 & 15 & $\mathrm{n} / \mathrm{m}$ & $\mathrm{n} / \mathrm{m}$ \\
\hline M11 & MST, VSL ground CST, medium sludge & 60 & 1 & 17 & 2 & 7.1 \\
\hline M11 & MST, VSL ground CST, medium sludge & 60 & 1 & 25 & $\mathrm{n} / \mathrm{m}$ & $\mathrm{n} / \mathrm{m}$ \\
\hline M11 & MST, VSL ground CST, medium sludge & 30 & 4 & 32 & 6 & 9.2 \\
\hline M11 & MST, VSL ground CST, medium sludge & 30 & 4 & 31 & $\mathrm{n} / \mathrm{m}$ & $\mathrm{n} / \mathrm{m}$ \\
\hline M11 & MST, VSL ground CST, medium sludge & 60 & 4 & 49 & 3.4 & 8.6 \\
\hline M11 & MST, VSL ground CST, medium sludge & 60 & 4 & 47 & $\mathrm{n} / \mathrm{m}$ & $\mathrm{n} / \mathrm{m}$ \\
\hline M2 & $\begin{array}{l}\text { MST, VSL ground CST } \\
\end{array}$ & 30 & 1 & 430 & 81 & 45 \\
\hline M2 & MST, VSL ground CST & 30 & 1 & 575 & $\mathrm{n} / \mathrm{m}$ & $\mathrm{n} / \mathrm{m}$ \\
\hline M2 & MST, VSL ground CST & 45 & 1 & 757 & 89 & 59 \\
\hline M2 & MST, VSL ground CST & 45 & 1 & 884 & $\mathrm{n} / \mathrm{m}$ & $\mathrm{n} / \mathrm{m}$ \\
\hline M2 & MST, VSL ground CST & 60 & 1 & 423 & 77 & 59 \\
\hline M2 & MST, VSL ground CST & 60 & 1 & 421 & $\mathrm{n} / \mathrm{m}$ & $\mathrm{n} / \mathrm{m}$ \\
\hline M2 & MST, VSL ground CST & 30 & 4 & 857 & 95 & 58 \\
\hline M2 & MST, VSL ground CST & 30 & 4 & 1101 & $\mathrm{n} / \mathrm{m}$ & $\mathrm{n} / \mathrm{m}$ \\
\hline M2 & MST, VSL ground CST & 45 & 4 & 984 & 102 & 73 \\
\hline M2 & MST, VSL ground CST & 45 & 4 & 922 & $\mathrm{n} / \mathrm{m}$ & $\mathrm{n} / \mathrm{m}$ \\
\hline M2 & MST, VSL ground CST & 60 & 4 & 383 & 109 & 71 \\
\hline M2 & MST, VSL ground CST & 60 & 4 & 510 & $\mathrm{n} / \mathrm{m}$ & $\mathrm{n} / \mathrm{m}$ \\
\hline M2 & MST, VSL ground CST & 30 & 10 & 1211 & 81 & 57 \\
\hline M2 & MST, VSL ground CST & 30 & 10 & 1281 & $\mathrm{n} / \mathrm{m}$ & $\mathrm{n} / \mathrm{m}$ \\
\hline M2 & MST, VSL ground CST & 45 & 10 & 829 & 92 & 64 \\
\hline
\end{tabular}


SRNL-STI-2011-00311, REV.0

\begin{tabular}{|c|c|c|c|c|c|c|}
\hline Feed & Feed Materials & $\begin{array}{l}\text { Temp } \\
\left({ }^{\circ} \mathrm{C}\right)\end{array}$ & $\begin{array}{c}\text { Time } \\
\text { (weeks) }\end{array}$ & $\begin{array}{c}\text { Shear Strength } \\
\text { (Pa) }\end{array}$ & $\begin{array}{l}\text { Yield Stress } \\
\text { (Pa) }\end{array}$ & $\begin{array}{c}\text { Consistency } \\
\text { (cP) }\end{array}$ \\
\hline M2 & MST, VSL ground CST & 45 & 10 & 737 & $\mathrm{n} / \mathrm{m}$ & $\mathrm{n} / \mathrm{m}$ \\
\hline M2 & MST, VSL ground CST & 60 & 10 & 412 & 91 & 64 \\
\hline M2 & MST, VSL ground CST & 60 & 10 & 350 & $\mathrm{n} / \mathrm{m}$ & $\mathrm{n} / \mathrm{m}$ \\
\hline M2 & MST, VSL ground CST & 30 & 13 & 1277 & 97 & 67 \\
\hline M2 & MST, VSL ground CST & 30 & 13 & 1231 & $\mathrm{n} / \mathrm{m}$ & $\mathrm{n} / \mathrm{m}$ \\
\hline M2 & MST, VSL ground CST & 45 & 13 & 574 & 101 & 70 \\
\hline M2 & MST, VSL ground CST & 45 & 13 & 826 & $\mathrm{n} / \mathrm{m}$ & $\mathrm{n} / \mathrm{m}$ \\
\hline M2 & MST, VSL ground CST & 60 & 13 & 474 & 83 & 59 \\
\hline M2 & MST, VSL ground CST & 60 & 13 & 529 & $\mathrm{n} / \mathrm{m}$ & $\mathrm{n} / \mathrm{m}$ \\
\hline M5 & MST, VSL ground CST, high sludge & 30 & 1 & 13 & 8.6 & 9.9 \\
\hline M5 & MST, VSL ground CST, high sludge & 30 & 1 & 14 & $\mathrm{n} / \mathrm{m}$ & $\mathrm{n} / \mathrm{m}$ \\
\hline M5 & MST, VSL round CST, high sludge & 45 & 1 & 3 & 2.9 & 7.8 \\
\hline M5 & MST, VSL ground CST, high sludge & 45 & 1 & 14 & $\mathrm{n} / \mathrm{m}$ & $\mathrm{n} / \mathrm{m}$ \\
\hline M5 & MST, VSL ground CST, high sludge & 60 & 1 & 25 & 3.2 & 8.9 \\
\hline M5 & MST, VSL ground CST, high sludge & 60 & 1 & 19 & $\mathrm{n} / \mathrm{m}$ & $\mathrm{n} / \mathrm{m}$ \\
\hline M5 & MST, VSL ground CST, high sludge & 30 & 4 & 23 & 5.8 & 9.1 \\
\hline M5 & MST, VSL ground CST, high sludge & 30 & 4 & 24 & $\mathrm{n} / \mathrm{m}$ & $\mathrm{n} / \mathrm{m}$ \\
\hline M5 & MST, VSL ground CST, high sludge & 45 & 4 & 31 & 3.2 & 8.7 \\
\hline M5 & MST, VSL ground CST, high sludge & 45 & 4 & 36 & $\mathrm{n} / \mathrm{m}$ & $\mathrm{n} / \mathrm{m}$ \\
\hline M5 & MST, VSL ground CST, high sludge & 60 & 4 & 47 & 2.8 & 7.9 \\
\hline M5 & MST, VSL ground CST, high sludge & 60 & 4 & 49 & $\mathrm{n} / \mathrm{m}$ & $\mathrm{n} / \mathrm{m}$ \\
\hline M5 & MST, VSL ground CST, high sludge & 30 & 10 & 37 & 5.5 & 9.1 \\
\hline M5 & MST, VSL ground CST, high sludge & 30 & 10 & 31 & $\mathrm{n} / \mathrm{m}$ & $\mathrm{n} / \mathrm{m}$ \\
\hline M5 & MST, VSL ground CST, high sludge & 45 & 10 & 53 & 3.1 & 8.9 \\
\hline M5 & MST, VSL ground CST, high sludge & 45 & 10 & 34 & $\mathrm{n} / \mathrm{m}$ & $\mathrm{n} / \mathrm{m}$ \\
\hline M5 & MST, VSL ground CST, high sludge & 60 & 10 & 60 & 3.9 & 8.7 \\
\hline M5 & MST, VSL ground CST, high sludge & 60 & 10 & 65 & $\mathrm{n} / \mathrm{m}$ & $\mathrm{n} / \mathrm{m}$ \\
\hline M5 & MST, VSL ground CST, high sludge & 30 & 13 & 41 & 6.2 & 9.3 \\
\hline M5 & MST, VSL ground CST, high sludge & 30 & 13 & 40 & $\mathrm{n} / \mathrm{m}$ & $\mathrm{n} / \mathrm{m}$ \\
\hline M5 & MST, VSL ground CST, high sludge & 45 & 13 & 52 & 3 & 8.5 \\
\hline M5 & MST, VSL ground CST, high sludge & 45 & 13 & 58 & $\mathrm{n} / \mathrm{m}$ & $\mathrm{n} / \mathrm{m}$ \\
\hline M5 & MST, VSL ground CST, high sludge & 60 & 13 & 57 & 4 & 9.1 \\
\hline M5 & MST, VSL ground CST, high sludge & 60 & 13 & 57 & $\mathrm{n} / \mathrm{m}$ & $\mathrm{n} / \mathrm{m}$ \\
\hline M7 & MST, high sludge & 60 & 4 & 42 & 3 & 7.9 \\
\hline M7 & MST, high sludge & 60 & 4 & 46 & $\mathrm{n} / \mathrm{m}$ & $\mathrm{n} / \mathrm{m}$ \\
\hline M7 & MST, high sludge & 60 & 10 & 63 & 3.1 & 8 \\
\hline M7 & MST, high sludge & 60 & 10 & 68 & $\mathrm{n} / \mathrm{m}$ & $\mathrm{n} / \mathrm{m}$ \\
\hline M7 & MST, high sludge & 60 & 13 & 79 & 3.2 & 8.3 \\
\hline M7 & MST, high sludge & 60 & 13 & 84 & $\mathrm{n} / \mathrm{m}$ & $\mathrm{n} / \mathrm{m}$ \\
\hline M8 & MST, VSL ground CST, low sludge & 30 & 1 & 143 & 25 & 24 \\
\hline M8 & MST, VSL ground CST, low sludge & 30 & 1 & 128 & ${ }^{\mathrm{n} / \mathrm{m}}$ & $\mathrm{n} / \mathrm{m}$ \\
\hline M8 & MST, VSL ground CST, low sludge & 45 & 1 & 109 & 19.6 & 23 \\
\hline M8 & MST, VSL ground CST, low sludge & 45 & 1 & 104 & $\mathrm{n} / \mathrm{m}$ & $\mathrm{n} / \mathrm{m}$ \\
\hline M8 & MST, VSL ground CST, low sludge & 60 & 1 & 153 & 18 & 22 \\
\hline M8 & MST, VSL ground CST, low sludge & 60 & 1 & 107 & $\mathrm{n} / \mathrm{m}$ & $\mathrm{n} / \mathrm{m}$ \\
\hline M8 & MST, VSL ground CST, low sludge & 30 & 4 & 118 & 27 & 26 \\
\hline
\end{tabular}


SRNL-STI-2011-00311, REV.0

\begin{tabular}{|c|c|c|c|c|c|c|}
\hline Feed & Feed Materials & $\begin{array}{l}\text { Temp } \\
\left({ }^{\circ} \mathrm{C}\right)\end{array}$ & $\begin{array}{c}\begin{array}{c}\text { Time } \\
\text { (weeks) }\end{array} \\
\end{array}$ & $\begin{array}{c}\text { Shear Strength } \\
\text { (Pa) }\end{array}$ & $\begin{array}{c}\text { Yield Stress } \\
(\mathbf{P a})\end{array}$ & $\begin{array}{c}\text { Consistency } \\
\text { (cP) }\end{array}$ \\
\hline M8 & MST, VSL ground CST, low sludge & 30 & 4 & 136 & $\mathrm{n} / \mathrm{m}$ & $\mathrm{n} / \mathrm{m}$ \\
\hline M8 & MST, VSL ground CST, low sludge & 45 & 4 & 112 & 22 & 28 \\
\hline M8 & MST, VSL ground CST, low sludge & 45 & 4 & 123 & $\mathrm{n} / \mathrm{m}$ & $\mathrm{n} / \mathrm{m}$ \\
\hline M8 & MST, VSL ground CST, low sludge & 60 & 4 & 149 & 19 & 24 \\
\hline M8 & MST, VSL ground CST, low sludge & 60 & 4 & 166 & $\mathrm{n} / \mathrm{m}$ & $\mathrm{n} / \mathrm{m}$ \\
\hline M8 & MST, VSL ground CST, low sludge & 30 & 10 & 155 & 21 & 24 \\
\hline M8 & MST, VSL ground CST, low sludge & 30 & 10 & 124 & $\mathrm{n} / \mathrm{m}$ & $\mathrm{n} / \mathrm{m}$ \\
\hline M8 & MST, VSL ground CST, low sludge & 45 & 10 & 139 & 20 & 26 \\
\hline M8 & MST, VSL ground CST, low sludge & 45 & 10 & 149 & $\mathrm{n} / \mathrm{m}$ & $\mathrm{n} / \mathrm{m}$ \\
\hline M8 & MST, VSL ground CST, low sludge & 60 & 10 & 252 & 16.1 & 21.8 \\
\hline M8 & MST, VSL ground CST, low sludge & 60 & 10 & 153 & $\mathrm{n} / \mathrm{m}$ & $\mathrm{n} / \mathrm{m}$ \\
\hline M8 & MST, VSL ground CST, low sludge & 30 & 13 & 121 & 24 & 23 \\
\hline M8 & MST, VSL ground CST, low sludge & 30 & 13 & 200 & $\mathrm{n} / \mathrm{m}$ & $\mathrm{n} / \mathrm{m}$ \\
\hline M8 & MST, VSL ground CST, low sludge & 45 & 13 & 202 & 29 & 33 \\
\hline M8 & MST, VSL ground CST, low sludge & 45 & 13 & 148 & $\mathrm{n} / \mathrm{m}$ & $\mathrm{n} / \mathrm{m}$ \\
\hline M8 & MST, VSL ground CST, low sludge & 60 & 13 & 137 & 23 & 26 \\
\hline M8 & MST, VSL ground CST, low sludge & 60 & 13 & 139 & $\mathrm{n} / \mathrm{m}$ & $\mathrm{n} / \mathrm{m}$ \\
\hline M10 & MST, VSL ground CST, 1:1 & 45 & 4 & 49 & 2.9 & 9.8 \\
\hline M10 & MST, VSL ground CST, 1:1 & 45 & 4 & 71 & $\mathrm{n} / \mathrm{m}$ & $\mathrm{n} / \mathrm{m}$ \\
\hline M12 & MST, VSL ground CST, sludge, 1:1:1 & 45 & 4 & 5 & 2.3 & 7.8 \\
\hline M12 & MST, VSL ground CST, sludge, 1:1:1 & 45 & 4 & 20 & $\mathrm{n} / \mathrm{m}$ & $\mathrm{n} / \mathrm{m}$ \\
\hline
\end{tabular}

Table 5. Average Rheological Properties as a Function of Feed Slurry with High (Red) and Low Values (Blue) Indicated

\begin{tabular}{|c|c|c|c|}
\hline Feed & Shear Strength (Pa) & \multicolumn{1}{|c|}{ Yield Stress (Pa) } & Consistency (cP) \\
\hline L1 & 765 & 13.1 & 35.1 \\
\hline L2 & 40 & 18.6 & $\mathbf{3 1 1}$ \\
\hline L3 & $\mathbf{2 0 1 9}$ & $\mathbf{9 1 . 4}$ & $\mathbf{7 . 2}$ \\
\hline L4 & 65 & 2.9 & 8.1 \\
\hline L5 & 22 & 3.6 & 10.2 \\
\hline L6 & 100 & 3.9 & 8.3 \\
\hline L7 & 122 & 3.4 & 16.0 \\
\hline L8 & 44 & 10.1 & 41.4 \\
\hline L9 & 458 & 24.8 & 9.7 \\
\hline M1 & 172 & $\mathbf{1 . 9}$ & 62.2 \\
\hline M2 & 749 & $\mathbf{9 1 . 5}$ & 8.8 \\
\hline M5 & 37 & 4.4 & 8.1 \\
\hline M7 & 64 & 3.1 & 25.1 \\
\hline M8 & 144 & 22.0 & 9.8 \\
\hline M10 & 60 & 2.9 & 8.8 \\
\hline M11 & 29 & 4.9 & 7.8 \\
\hline M12 & $\mathbf{1 2}$ & 2.3 & M1 = MST \\
\hline L1 = MST & \multicolumn{2}{|c|}{ MST + SRNL ground CST (1:17 ratio) } \\
L3= MST + unground CST (1:17 wt ratio) & M2= MST + VSL ground CST (1:17 wt ratio) \\
\hline
\end{tabular}




\begin{tabular}{|l|l|}
\hline L4 $=$ MST + Sludge (1:1.5 wt ratio) & M7 = MST + Sludge (1:660) \\
L5 = MST + Sludge + SRNL ground CST (1:660:17 ratio) & M8 = MST + Sludge + VSL ground CST (1:1.5:17 ratio) \\
L6 = MST + Sludge + unground CST (1:660:17 ratio) & M10 = MST + VSL ground CST (1:1 ratio) \\
L7 = MST + Sludge (1:660) & M11 = MST + Sludge + VSL ground CST (1:82:17 ratio) \\
L8 = MST + Sludge + SRNL ground CST (1:1.5:17 ratio) & M12 = MST + Sludge + VSL ground CST (1:1:1 ratio) \\
L9 = MST + Sludge + unground CST (1:1.5:17 ratio) & \\
\hline
\end{tabular}

For many of the slurries, the maximum shear strength was observed after settling for 13 weeks at $60^{\circ} \mathrm{C}$. These slurries are L1, L3, L6, L7, L9, and M7. With the M2 slurry, the maximum shear strength was observed after settling for $10-13$ weeks at $30^{\circ} \mathrm{C}$. The maximum yield stress and consistency were observed at 13 weeks and $60^{\circ} \mathrm{C}$ for slurries L1, L6, and M7.

The authors performed analyses to determine the impact of feed slurry, settling time, settling temperature, CST type, CST concentration, and sludge concentration on the slurry shear strength. Statistical analyses were completed using JMP analytical software and significance was based on a 95\% confidence level. ${ }^{9}$ A discussion of the results follows.

Figure 4 shows a plot of the natural log of the shear strength as a function of the sludge concentration for all samples that settled for four weeks at $30^{\circ} \mathrm{C}$. A range of 2.3 to 4.6 on the $\mathrm{Y}$ axis is equivalent to shear strengths of 10 to $100 \mathrm{~Pa}$. The plot shows that the shear strength decreases as a function of sludge concentration in the solids. Similar results were observed at other settling times and temperatures. Statistical analysis of the data shows the effect to be statistically significant.

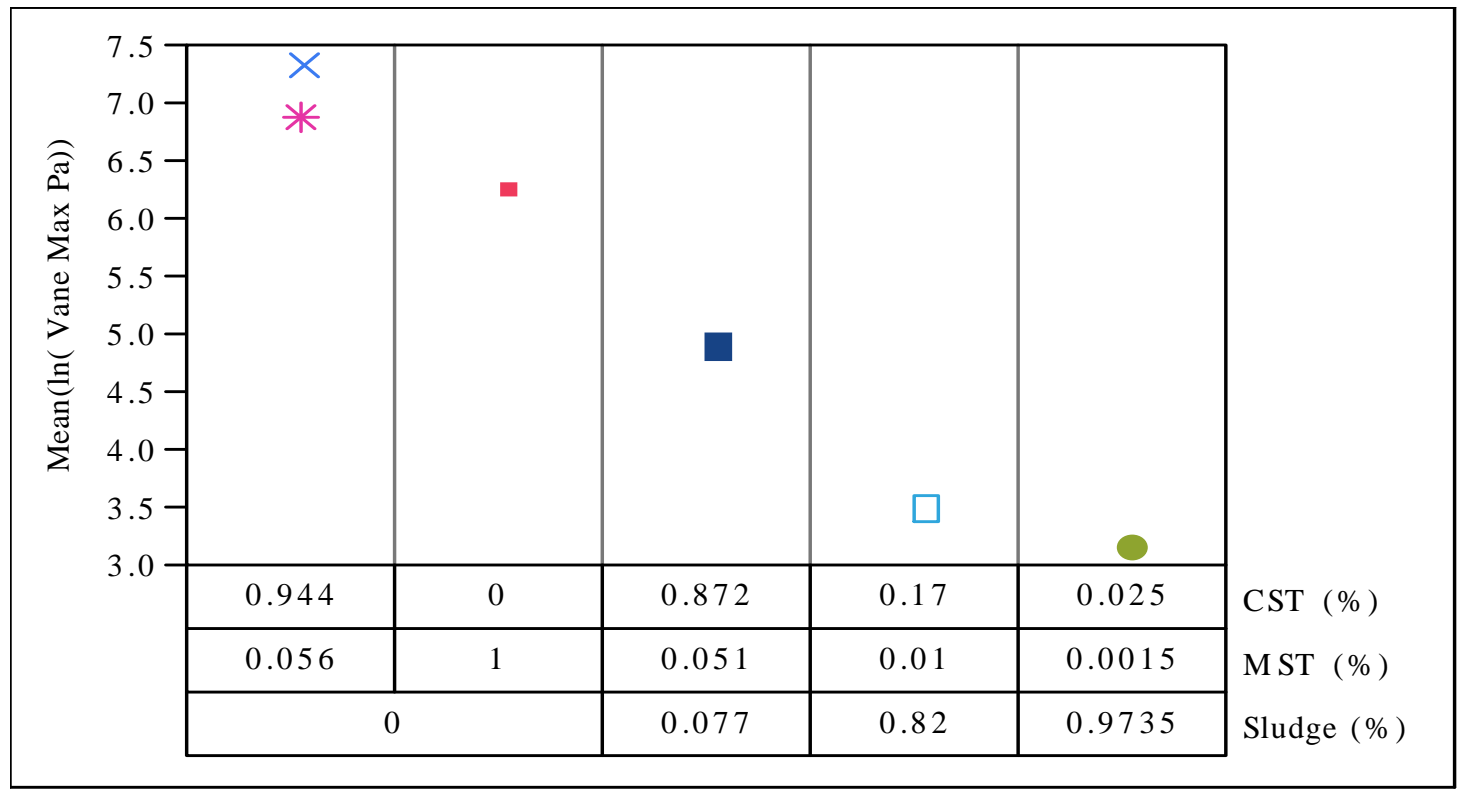

Figure 4. Shear Strength as a Function of Sludge Concentration for 4 Week, $30^{\circ} \mathrm{C}$ Samples

Figure 5 shows the natural log of the shear strength as a function of the CST concentration for all samples that settled for one week at $60^{\circ} \mathrm{C}$. The plot shows the shear strength increases with CST concentration. In addition, the plot shows unground CST produces the largest shear strength followed by VSL ground CST. Similar results were observed at other settling times and temperatures. Statistical analysis of the data shows the effect to be statistically significant. 


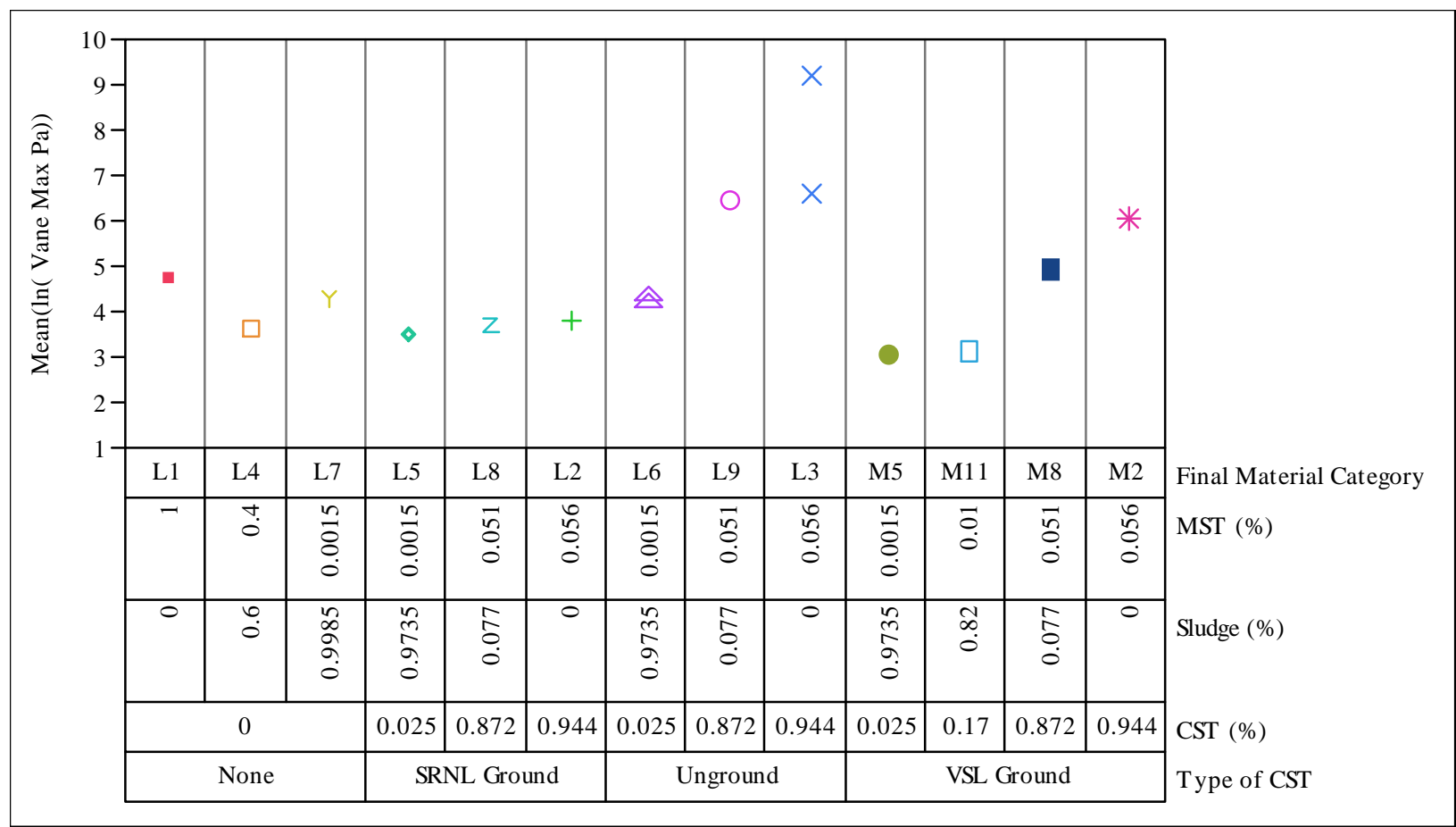

Figure 5. Shear Strength as a Function of CST Concentration for One Week, $60^{\circ} \mathrm{C}$ Samples

The authors conducted a similar review to evaluate the impact of MST concentration on the slurry shear strength. The analysis showed no statistically significant effects correlating MST concentration to the slurry shear strength.

Figure 6 shows the shear strength as a function of time and temperature for the MST only feed slurries (L1 and M1). From the plot, the L1 and M1 feed materials appear to have similar shear strengths, and statistical analysis of the data shows that there is no statistically significant difference between the two feeds. This supports the conclusion that the two MST materials are effectively equivalent and that samples containing different MST batches may be compared. Sample 57 appears to be an outlier. A repeat of the test conditions (sample 119) shows shear strength measurements that are more consistent with the other data. If the outlier is removed, statistical analysis shows the shear strength to be a function of settling time, but not a function of settling temperature.

Figure 6 data indicate the measured shear strength of the MST samples that settled for four weeks at $45^{\circ} \mathrm{C}$ to be 105 and $118 \mathrm{~Pa}$. Preliminary pilot-scale resuspension test results show that MST only slurries can be resuspended with three submersible mixer pumps ${ }^{\mathrm{d}}$ (SMPs) after settling under these conditions ( 4 weeks at $\left.45^{\circ} \mathrm{C}\right) .{ }^{10}$ Allowing the MST to settle for an additional four weeks (eight weeks total) during rheology testing led to increased shear strengths of 194 to $351 \mathrm{~Pa}$; thus, it can be predicted from this data that pilot-scale or full-scale operation with eight week settling times will be more challenging to mix. Pilot-scale testing showed that slurries containing MST and ground CST (similar to L2 and M2) could be resuspended with the

\footnotetext{
${ }^{\mathrm{d}}$ The submersible mixer pump is the planned mixing pump for SCIX. Pilot-scale testing used three scaled submersible mixer pumps.
} 
equivalent of three SMPs. ${ }^{11}$ Given that the MST and ground CST that settled for four weeks at $45^{\circ} \mathrm{C}$ had a larger shear strength $(922-984 \mathrm{~Pa})$ than the MST slurries that settled for eight weeks at $45{ }^{\circ} \mathrm{C}(194-351 \mathrm{~Pa})$, three SMPs should be able to resuspend MST slurries that settle for eight weeks at $45^{\circ} \mathrm{C}$. The authors recommend conducting additional resuspension tests if SCIX wishes to allow the MST to settle for more than four weeks.

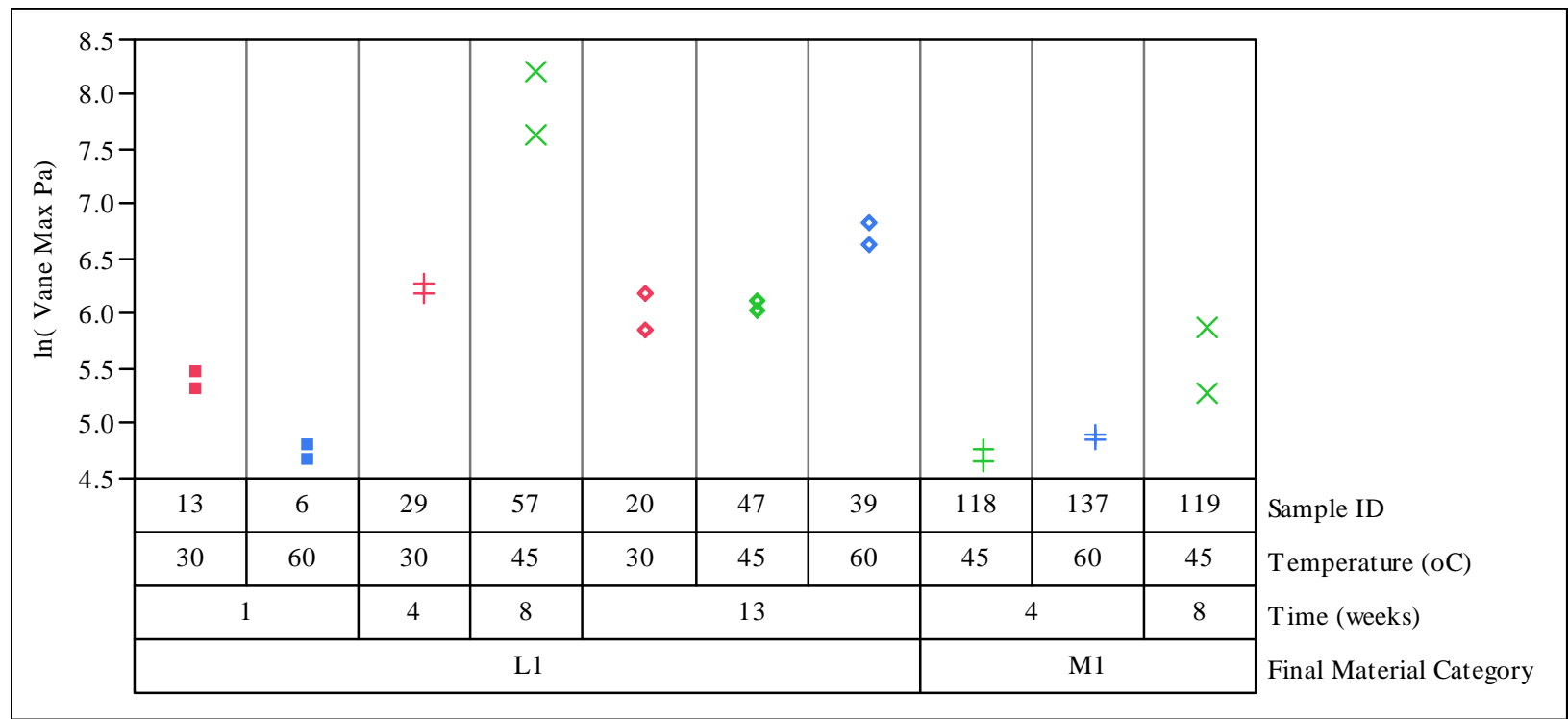

Figure 6. Shear Strength of MST only Slurries as a function of Time and Temperature

Figure 7 shows the shear strength as a function of time and temperature for the MST plus ground CST feed slurries (L2 and M2). Statistical analysis of the data shows the feed with VSL ground CST (M2) has a higher shear strength than the feed with SRNL ground CST (L2). No statistically significant effect of settling time on the shear strength of these materials is noted; however, the statistical analysis shows a temperature effect, in which shear strength decreases with increased temperature. These results differ from previous test data collected with MST and sludge mixtures, as well as other results collected in this study, indicating that CST may have a unique effect on rheological properties in this mixture. ${ }^{2,3}$ 


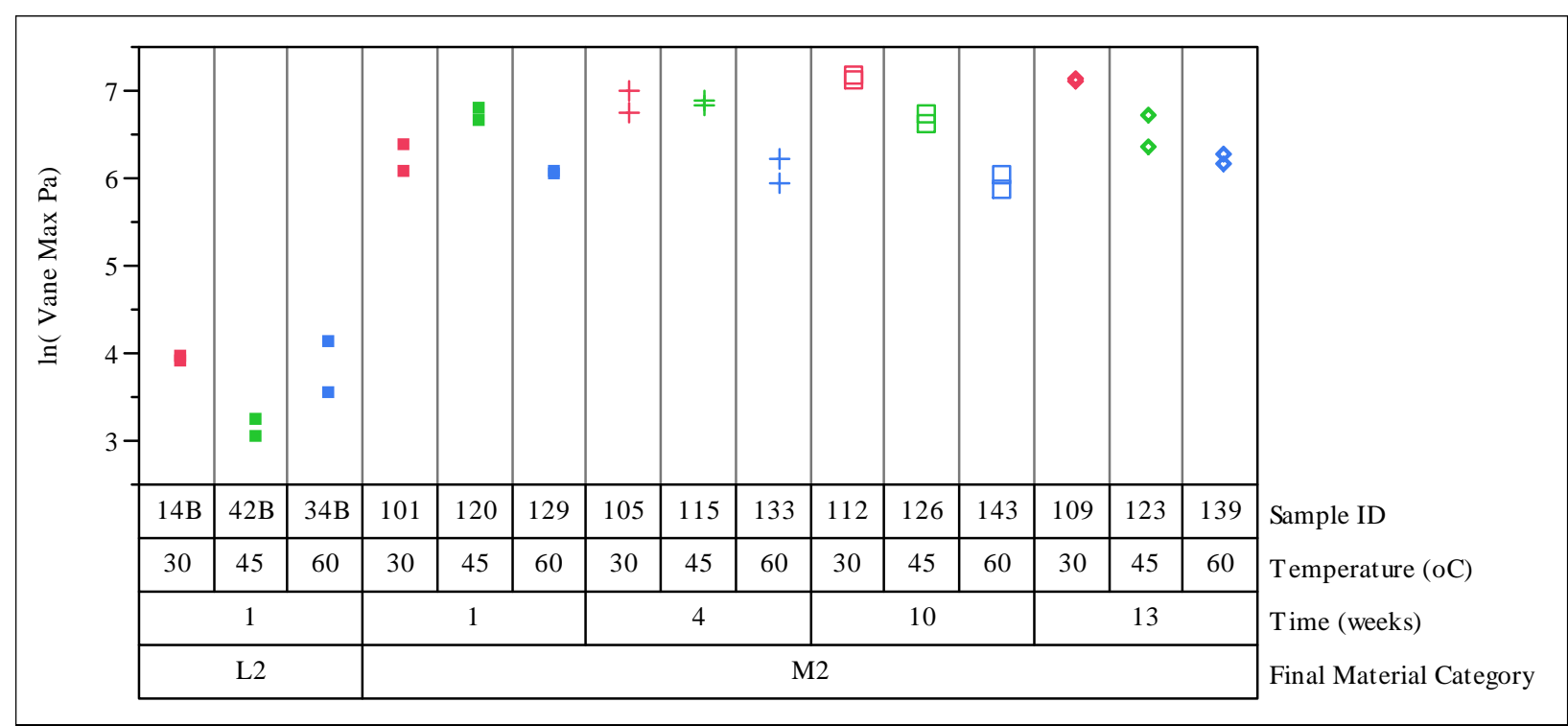

Figure 7. Shear Strength of MST Plus Ground CST Slurries as a function of Time and Temperature

Pilot-scale resuspension tests showed that greater than $99.9 \%$ of the solids in an MST and VSL ground CST slurry that settled for four weeks at $45^{\circ} \mathrm{C}$ could be resuspended with three SMPs. The ability to resuspend MST-CST slurries with shear strength as large as 922 to 984 Pa suggests that three SMPs may be able to suspend MST slurries that settled for more than four weeks. If there is consideration for longer settling times during SCIX operations, testing is recommended to confirm resuspension capability.

Figure 8 shows the shear strength as a function of time and temperature for the MST plus unground CST feed slurries (L3). The data show no time or temperature effects. However, all of the samples showed large shear strength $(>400 \mathrm{~Pa})$, which would make resuspending this material challenging. In addition, the large particle size ( 400 micron) would also make resuspension difficult. 


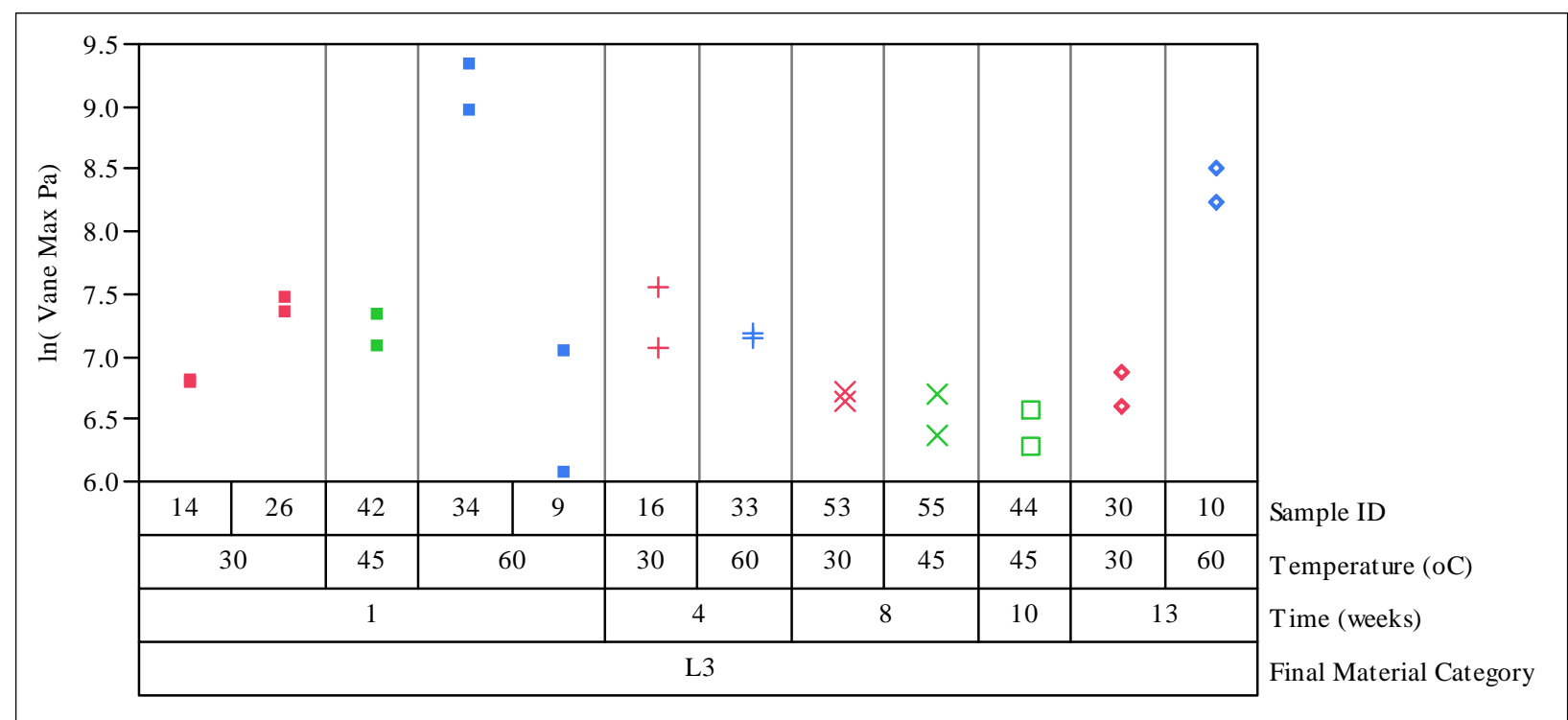

Figure 8. Shear Strength of MST Plus Unground CST Slurries as a function of Time and Temperature

Figure 9 shows the shear strength as a function of time and temperature for the MST plus low sludge feed slurries (L4). Statistical analysis of the data shows the shear strength to be a function of settling temperature, but not a function of settling time.

If the loaded CST is not transferred into Tank $41 \mathrm{H}$ as a part of the SCIX flowsheet, the L1 and L4 feeds bound the expected composition, and become the best representation of solid particle behavior in Tank 41H. Given that the shear strength of the MST plus low sludge slurry is much less than the shear strength of the MST only, and that the MST only slurry which sat for 4 weeks at $45^{\circ} \mathrm{C}$ were resuspended with 3 SMPs, low sludge and MST particles that settle for 4 weeks at $45^{\circ} \mathrm{C}$ should be able to be resuspended with 3 SMPs. The slurries likely could settle for a longer time or at higher temperatures and still reach full suspension with this setup, but additional testing would be recommended. 


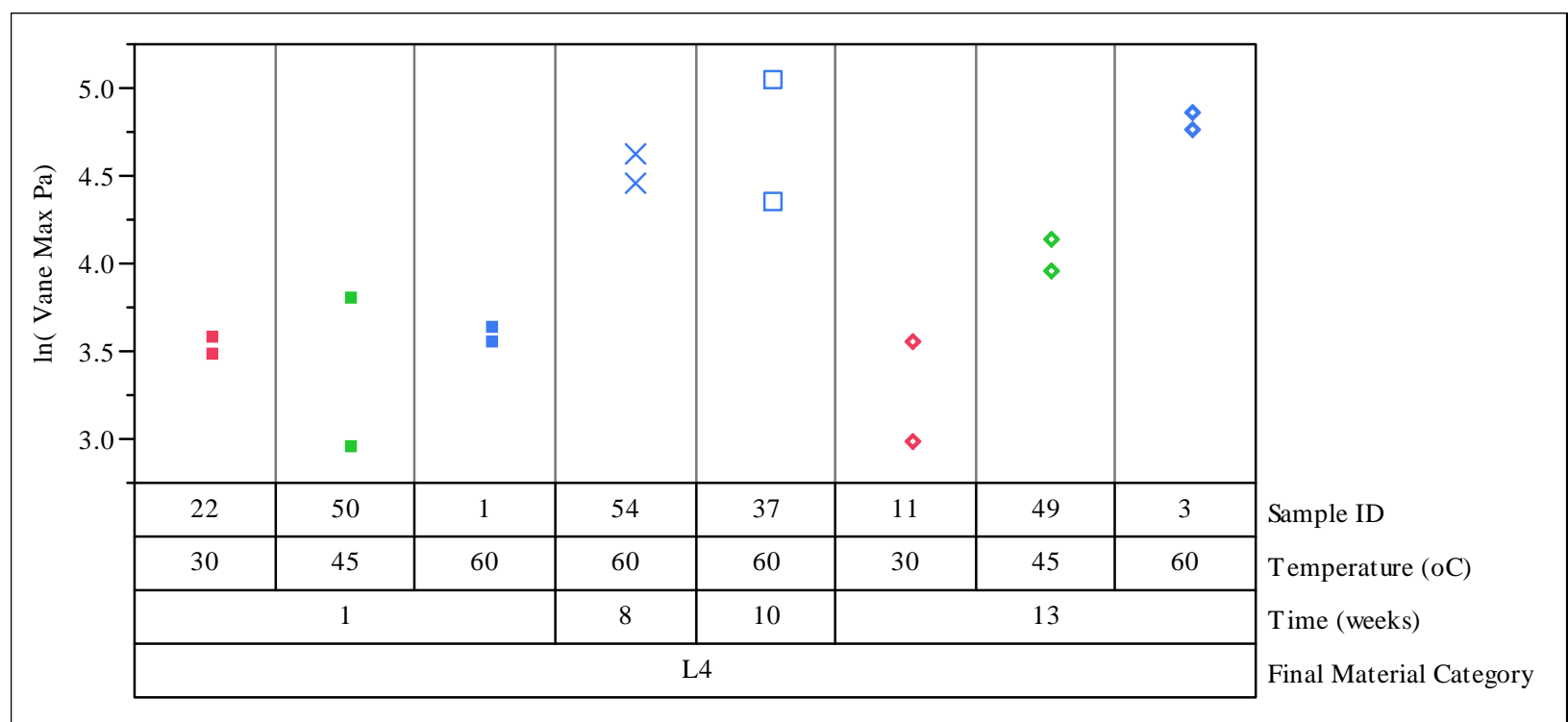

Figure 9. Shear Strength of MST Plus Low Sludge Slurries as a function of Time and Temperature

Figure 10 shows the shear strength as a function of time and temperature for the MST plus ground CST plus high sludge feed slurries (L5 and M5). This feed is representative of projected conditions in Tank 40H. Statistical analysis of the data shows no difference between the L5 and M5 samples. The analysis shows very strong effects of settling time and temperature on the slurry shear strength, with the time effect being more significant.

Comparing shear strengths observed in the M5 feed after four weeks at $45^{\circ} \mathrm{C}$ to those observed in M1 shows that 3 SMPs should be sufficient to suspend this material. Tank $40 \mathrm{H}$ employs 4 quad volute pumps rather than SMPs. This pilot scale test is currently underway to evaluate the ability in Tank 40H to resuspend M5 feeds. Results of this test will be documented in a separate report.

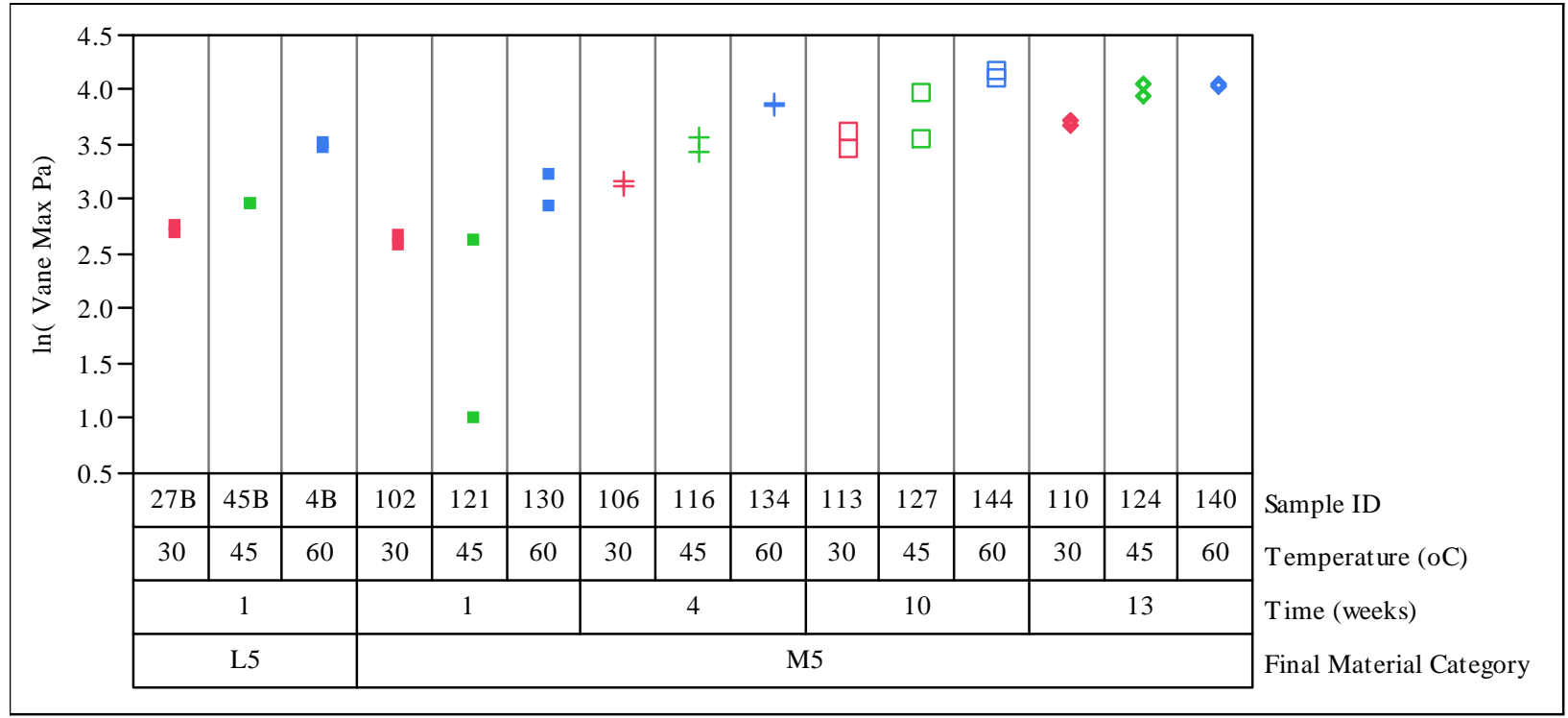


Figure 10. Shear Strength of MST Plus Ground CST Plus High Sludge Slurries as a function of Time and Temperature

Figure 11 shows the shear strength as a function of time and temperature for the MST plus unground CST plus high sludge feed slurries (L6). The analysis shows a strong effect of settling time and temperature on the slurry shear strength. Statistical analysis shows L6 to be different from M5, indicating that unground CST produces large shear strength, even when present in low concentrations. Further, it shows that the CST preparation introduced into a feed can affect even slurries in which another material is the prevailing component, e.g. sludge solids.

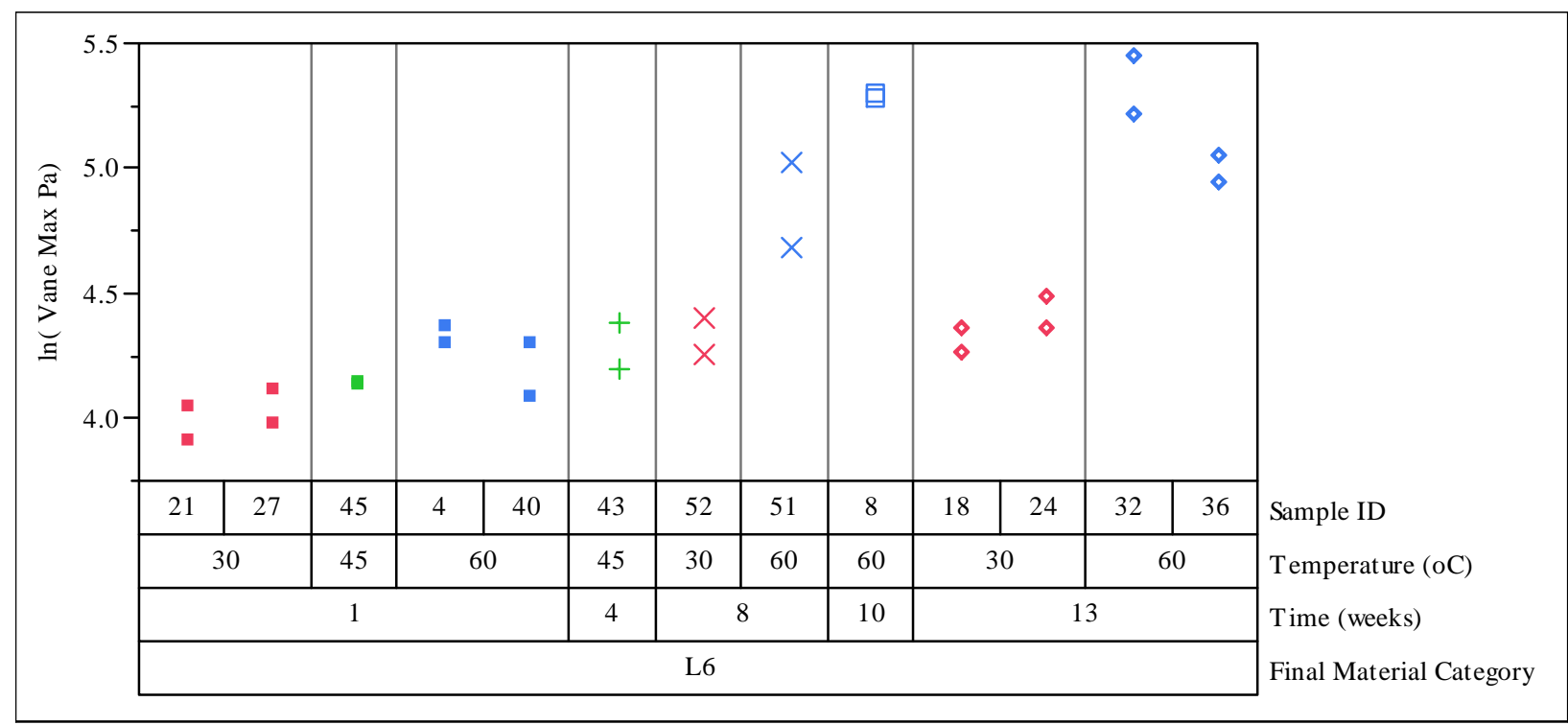

Figure 11. Shear Strength of MST Plus Unground CST Plus High Sludge Slurries as a function of Time and Temperature

The maximum shear strength observed in the testing of this feed was $223 \mathrm{~Pa}$. Given that resuspension was attainable with $3 \mathrm{SMPs}$ in pilot scale testing of $45^{\circ} \mathrm{C}$ material settled for 4 weeks, it can be inferred that resuspension of sludge, MST, and CST mixtures below similar shear strengths could also be reached. Data indicate that this pump setup may be able to resuspend mixtures that have settled longer than 4 weeks or at temperatures greater than $45^{\circ} \mathrm{C}$. However, the large particle size of the unground CST may make it difficult to resuspend with 3 SMPs. Further evaluations and testing with longer settling times are recommended if addition of unground CST to a waste tank is required (not planned as part of SCIX operations).

Figure 12 shows the shear strength as a function of time and temperature for the MST plus high sludge feed slurries (L7 and M7). Feeds L7 and M7 are statistically indistinguishable despite separate MST sources, and settling time and temperature have a negligible effect on slurry shear strength.

All MST plus high sludge samples displayed shear strengths of $<100 \mathrm{~Pa}$, with the exception of that held for 13 weeks at $60^{\circ} \mathrm{C}$. These shear strengths are less than MST only feeds, which have been demonstrated to be capable of resuspension by three SMPs based on tests performed at $45^{\circ} \mathrm{C}$ after 4 weeks of settling. ${ }^{11}$ This case is representative of Tank $42 \mathrm{H}$ and $51 \mathrm{H}$. Tank $42 \mathrm{H}$ 
contains four standard pumps and Tank $51 \mathrm{H}$ contains four quad volute pumps. As indicated with the previous feed, Tank $40 \mathrm{H}$ employs 4 quad volute pumps rather than 3 SMPs. This resuspension testing, to evaluate mixing in Tanks $40 \mathrm{H}, 42 \mathrm{H}$, and $51 \mathrm{H}$, has been performed at the pilot scale facility.

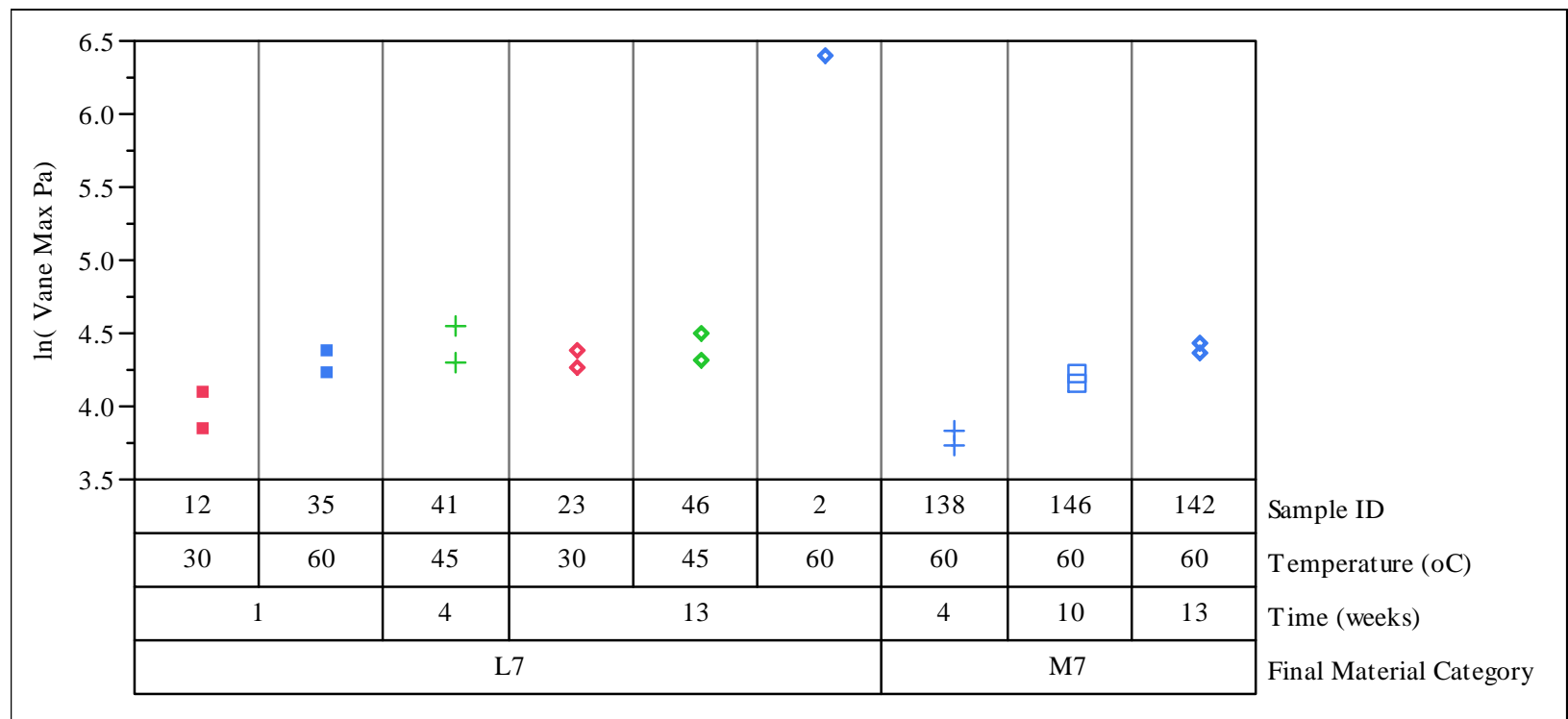

Figure 12. Shear Strength of MST Plus High Sludge Slurries as a function of Time and Temperature

Figure 13 shows the shear strength as a function of time and temperature for the MST plus ground CST plus low sludge feed slurries (L8 and M8). The analysis shows that the SRNL ground CST (L8) behaves differently than the VSL ground CST (M8), producing lower shear strengths. This observation mirrors what was found in the L2 and M2 feeds, but differences are notably stronger. With feed slurry M8, settling time has a statistically significant effect, but the data are very noisy. No temperature effect was observed.

The higher shear strengths observed in VSL ground CST compared to SRNL ground CST may be explained by the grinding processes and the average particle sizes resultant in each material. VSL ground CST was processed for a much shorter grinding time than SRNL ground materials and maintained larger particle sizes. In addition, the SRNL ground material was stored in drums outside for approximately five years. In that time, some of the binder and other chemical compounds that produce cohesive slurries may have leached from the CST, resulting in the lower shear strength. Larger, heavier CST particles tend to settle more rapidly within the feed, resulting in a greater difficulty in mixing and resuspension. Smaller CST particles, such as those found in the SRNL ground material, tend to stay suspended within the mixture and are less prone to create problematic shear strengths. 


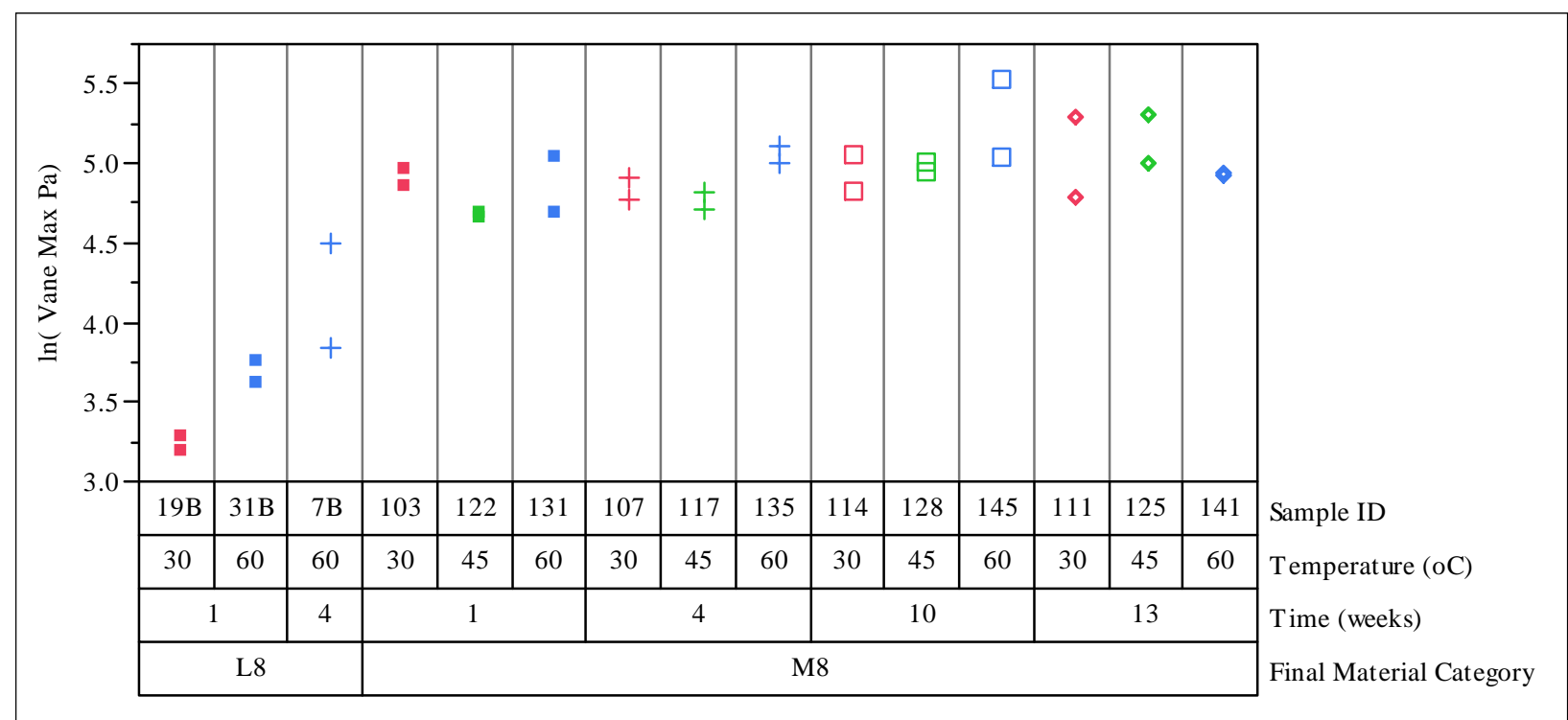

Figure 13. Shear Strength of MST Plus Ground CST Plus Low Sludge Slurries as a function of Time and Temperature

Figure 14 shows the shear strength as a function of time and temperature for the MST plus unground CST plus low sludge feed slurries (L9). Data indicate a large increase in shear strengths as compared to those observed in similar feeds containing ground CST (L8, M8). Statistical analysis of the L9 data shows no effects of settling time or temperature on the slurry shear strength. However, the data show an unusually large variance between replicates, up to five times larger than that observed in all other feeds. One plausible explanation for the observed variability in this feed is that it may be an effect of the formation of a heterogeneous layer of a single material within the settled solids, which interfered with vane measurements. Layer formation is discussed further in Section 4.4 of this report.

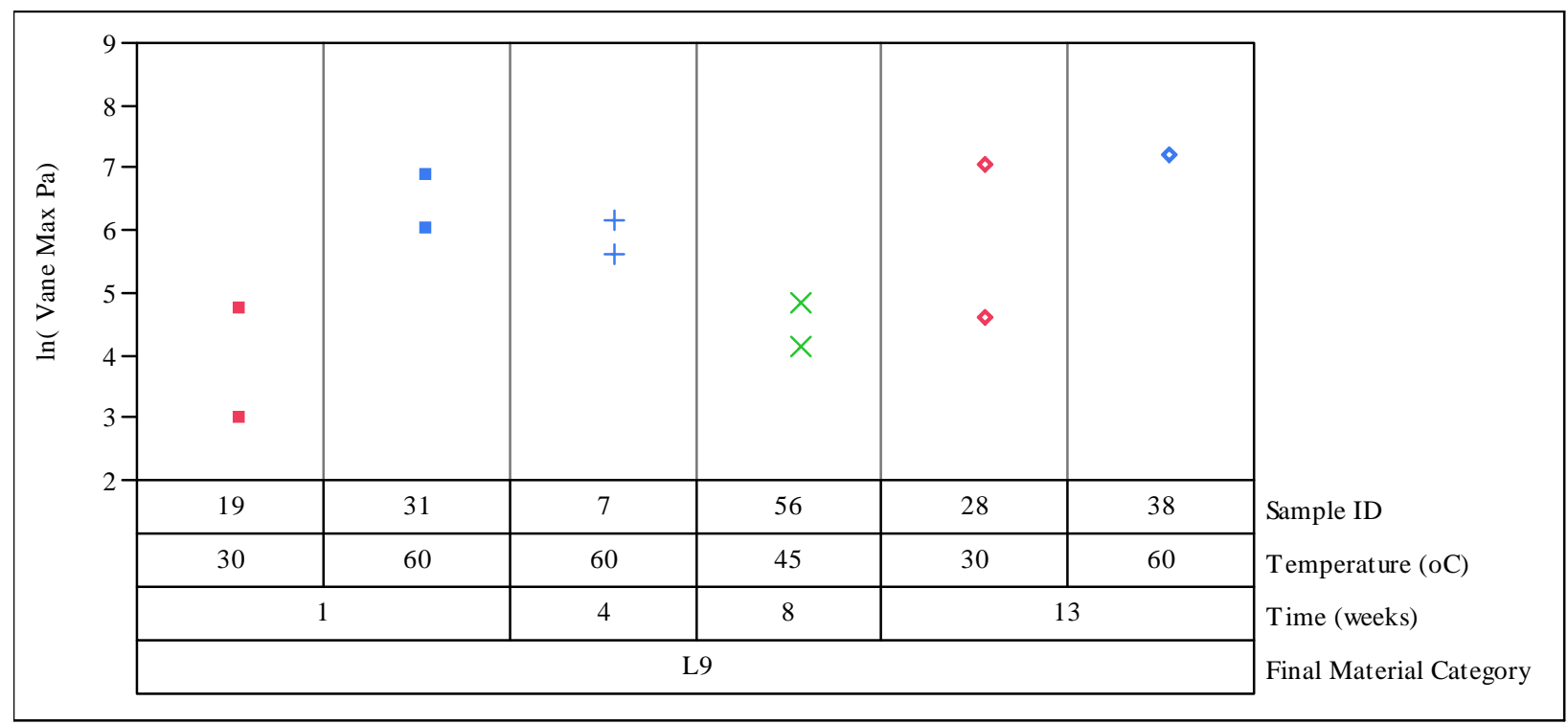

Figure 14. Shear Strength of MST Plus Unground CST Plus Low Sludge Slurries as a function of Time and Temperature 
Feed M10 is not discussed herein because samples tested provided insufficient data points for statistical analysis or meaningful plot depiction.

Figure 15 shows the shear strength as a function of time and temperature for the MST plus ground CST plus medium sludge feed slurries (M11). Statistical analysis of the data shows that settling time and temperature are significant and influence the slurry shear strength.

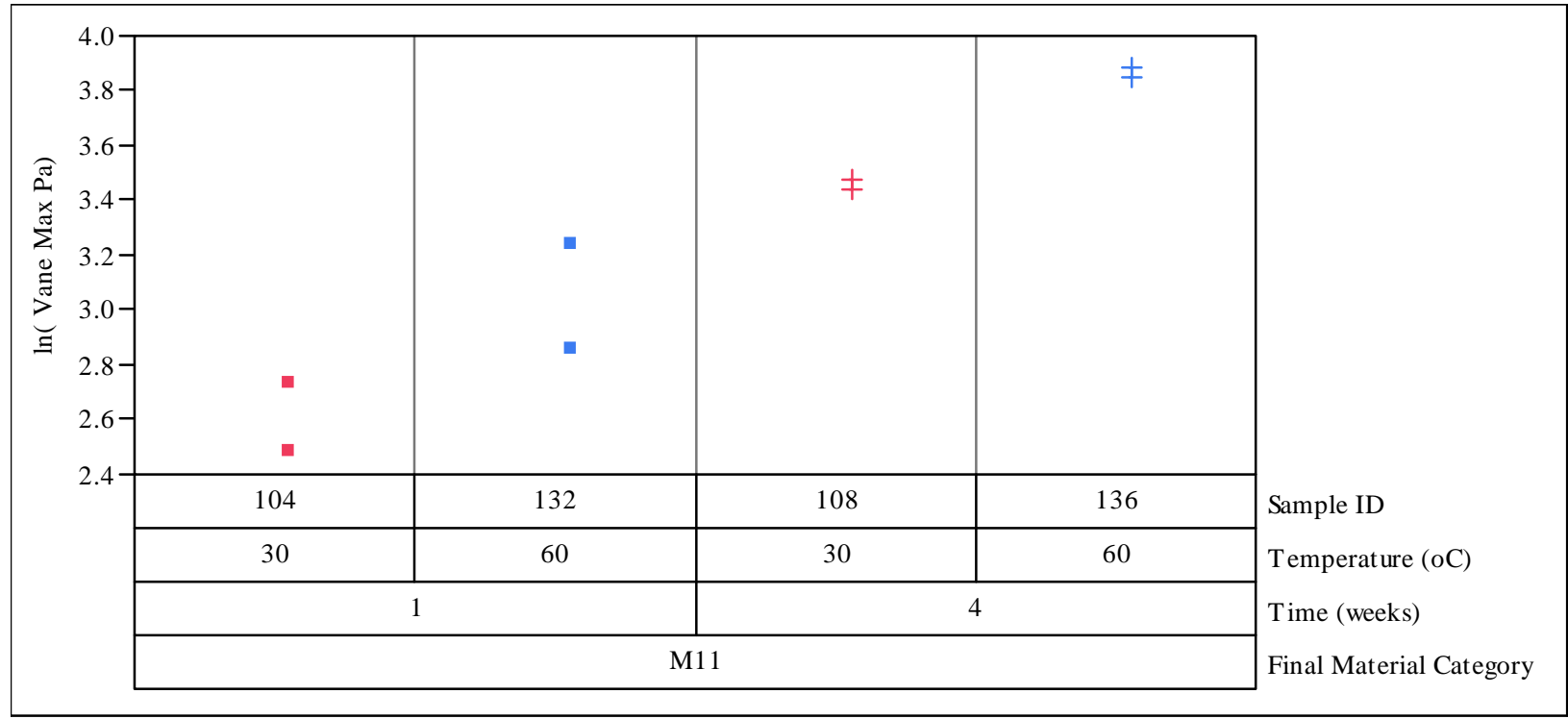

Figure 15. Shear Strength of MST Plus Ground CST Plus Medium Sludge Slurries as a function of Time and Temperature

Feed M12 is not discussed herein because samples tested provided insufficient data points for statistical analysis or meaningful plot depiction.

All of the feed slurries containing MST, CST, and high sludge or medium sludge showed effects of settling time and temperature on the shear strength. Both feed slurries containing only MST showed settling time effects on shear strength. Feed M8, which contains MST, ground CST, and low sludge, showed an effect of settling time on the shear strength.

\subsection{FLOW CURVE MEASUREMENTS}

Table 4 shows the measured yield stress and consistency as a function of feed slurry, settling time, and settling temperature. Table 5 shows the average yield stress and average consistency as a function of feed slurry. The highest yield stress and consistency samples appear to be those containing MST and unground CST (L3) and MST and VSL ground CST (M2) in a 1:17 ratio. The average yield stress and consistency of the MST plus unground CST samples are 91.4 Pa and $311 \mathrm{cP}$, respectively. The average yield stress and consistency of the MST and VSL ground CST samples are $91.5 \mathrm{~Pa}$ and $62.2 \mathrm{cP}$, respectively. The yield stress is dominating the rheological behavior of M2 and L3, and both hover in the $90 \mathrm{~Pa}$ range which is well outside the range for inter tank transfers. The slurries had a larger insoluble solids concentration than typical 
$\mathrm{H}$-area to SWPF transfers (12 to $18 \mathrm{wt} \%$ insoluble solids). Decreasing the insoluble solids concentration will reduce the yield stress to a level that is acceptable for inter-area transfer.

Figure 16 shows the yield stress as a function of CST concentration for samples that settled for 13 weeks at $30^{\circ} \mathrm{C}$. The plot shows that yield stress increases with increasing CST concentration in the slurry. In addition, for slurries with no CST, the yield stress statistically decreases with increasing sludge concentration. These trends are the same as the trends observed in the shear strength measurements. Similar trends were observed at other settling times and temperatures.

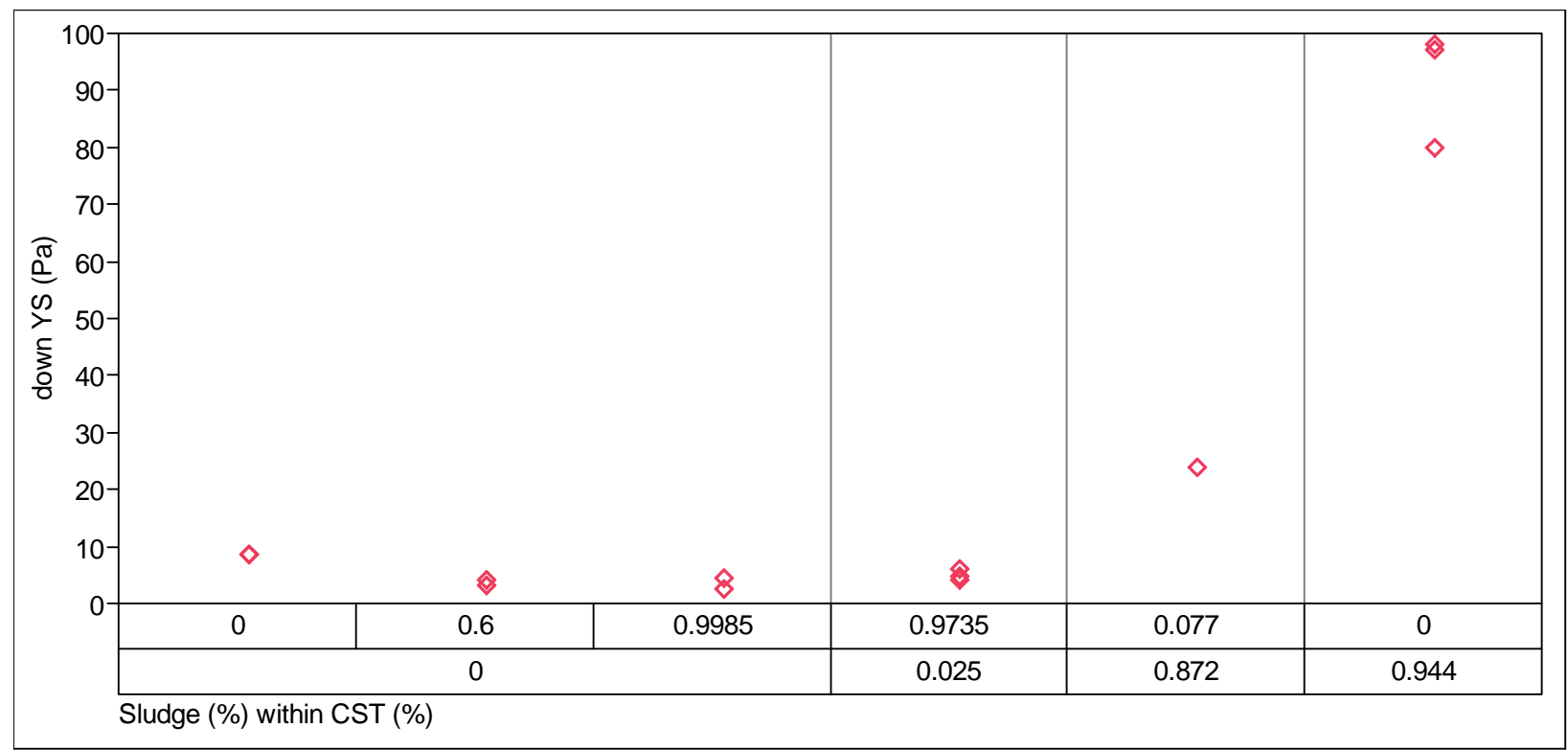

Figure 16. Yield stress as a Function of Unground CST concentration for $13 \mathrm{Week}, 30^{\circ} \mathrm{C}$ Samples

Figure 17 shows the consistency as a function of CST concentration for samples that settled for 13 weeks at $30^{\circ} \mathrm{C}$. The plot shows that consistency increases with increasing CST concentration in the slurry. In addition, for slurries with no CST, the consistency statistically decreases with increasing sludge concentration. These trends are the same as the trends observed in the shear strength measurements. Similar trends were observed at other settling times and temperatures. 


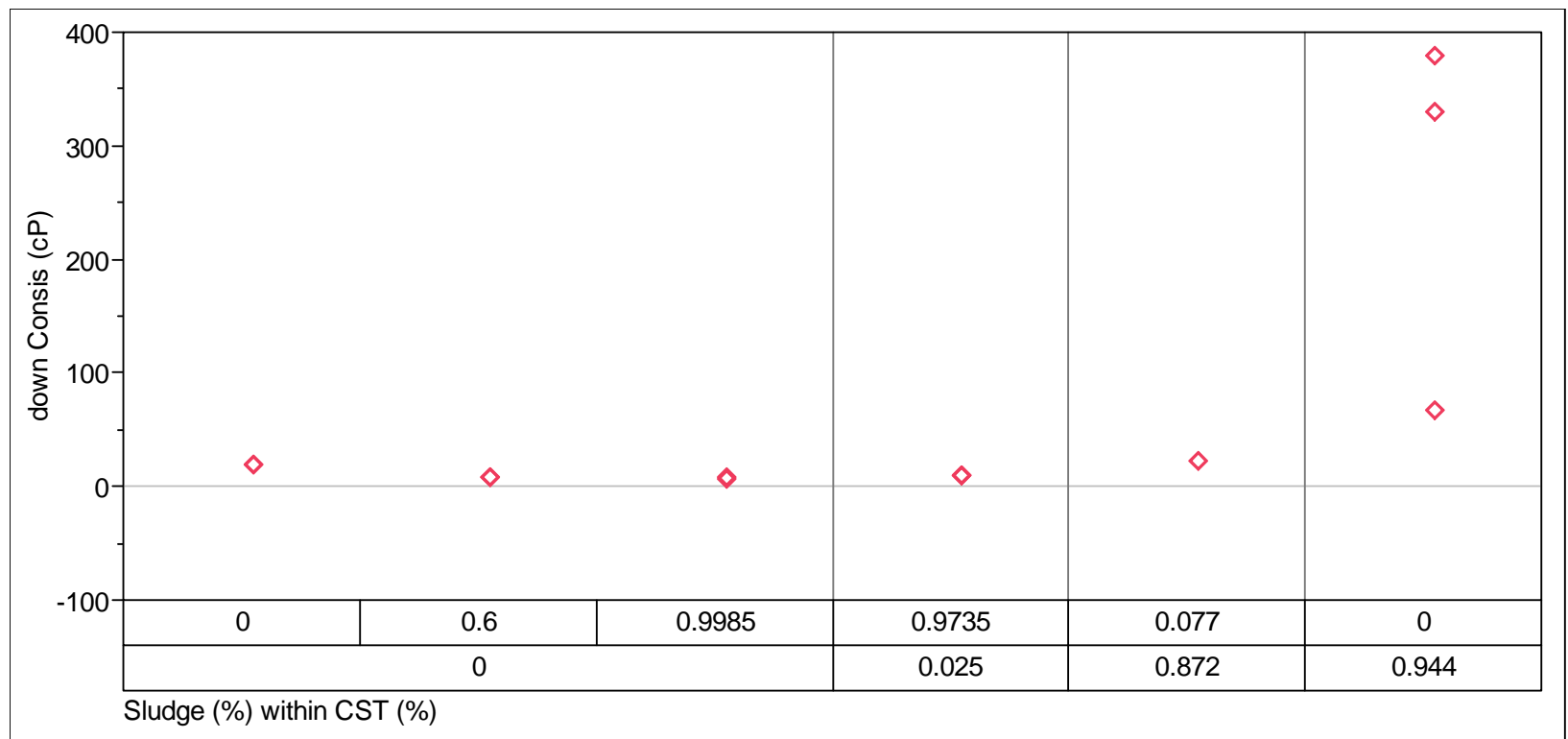

Figure 17. Consistency as a Function of Unground CST concentration for 13 Week, $30^{\circ} \mathrm{C}$ Samples

Figure 18 shows the yield stress as a function of settling time and temperature for slurries containing only MST (i.e., L1 and M1). The plot suggests that settling time and temperature do not have an effect on the slurry yield stress. The data also indicate that the second set of samples (M1) had a statistically significant lower yield stress than the first set (L1). The insoluble solids for M1 are all less than 18\%, while L1 insoluble solids range from 17 to $21 \mathrm{wt} \%$; this may explain this observation. Sample 39 was exposed to the highest temperature and had the longest settling time; the combination of time and temperature may explain the large increase in yield stress for this sample. Remaining data points demonstrate yield stresses less than $15 \mathrm{~Pa}$.

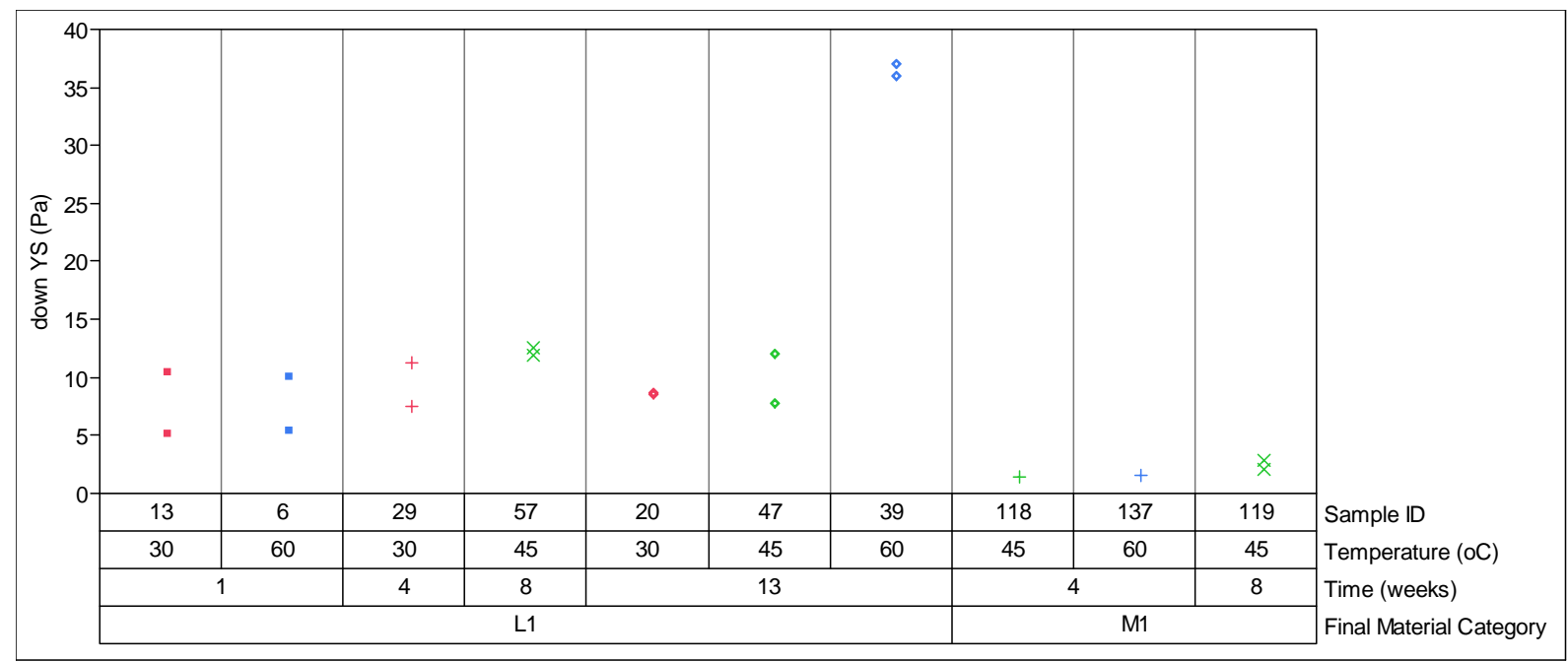

Figure 18. Yield Stress of MST only Slurries as a Function of Time and Temperature

Figure 19 shows the consistency as a function of settling time and temperature for slurries containing only MST (i.e., L1 and M1). The plot suggests that settling time and temperature do not have an effect on the slurry consistency. The data also indicate that the second set of 
samples (M1) had a statistically significant lower consistency than the first set (L1). Sample 39 was exposed to the highest temperature and had the longest settling time; the combination of time and temperature may explain the large increase in consistency for this sample. Remaining data points demonstrate consistencies less than $40 \mathrm{cP}$.

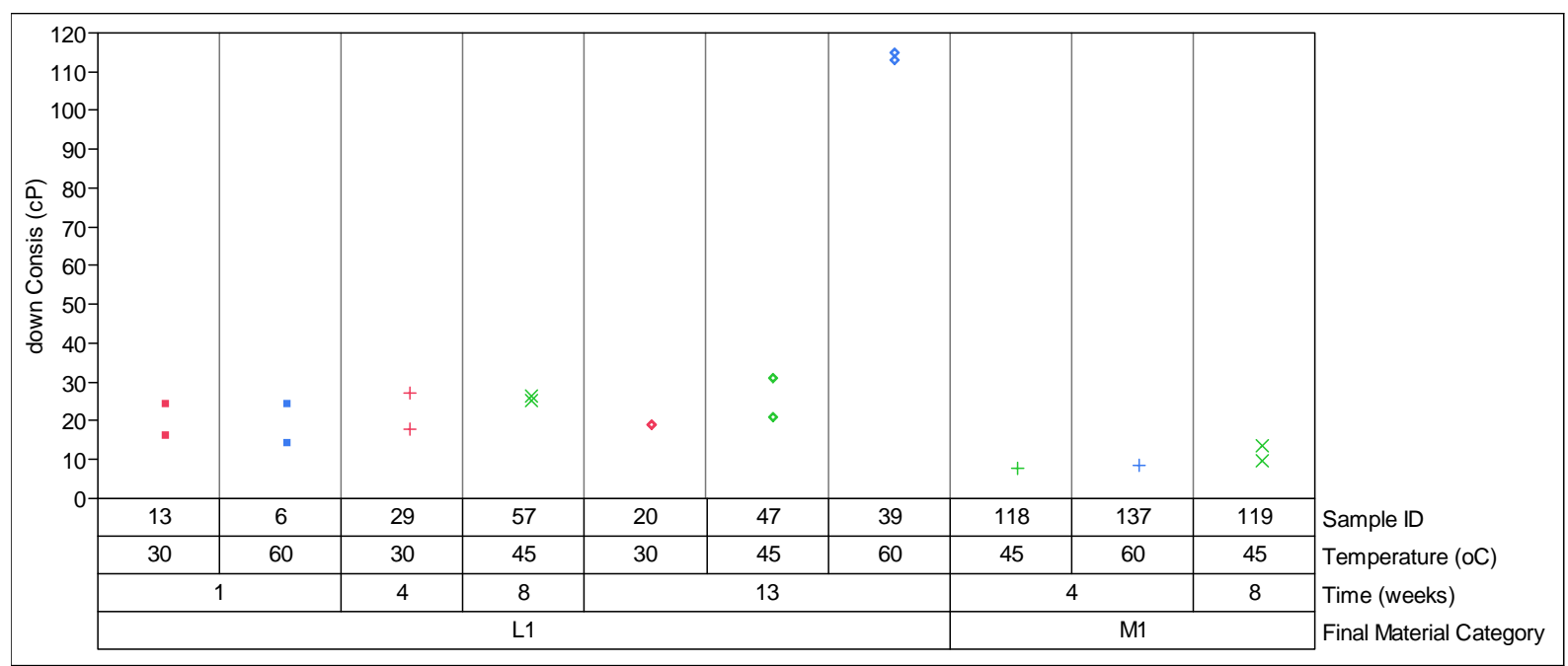

Figure 19. Consistency of MST only Slurries as a Function of Time and Temperature

Figure 20 shows the slurry yield stress as a function of settling time and temperature for slurries containing MST and ground CST (1:17 ratio). The plot suggests that settling time and temperature do not have an effect on the slurry yield stress. The data also indicate that the second set of samples (M2) had a higher yield stress than the first set (L2); this data is statistically significant. Slurries with VSL ground CST (M2) also had statistically significant higher shear strengths than equivalent slurries with SRNL ground CST (L2).

Typical yield stress measurements of MST plus VSL ground CST material average 100 Pa. Yield stresses measured in the M2 feed samples would exceed the criterion for $\mathrm{H}$ to $\mathrm{S}$ Area transfers (3 to $10 \mathrm{~Pa}$ yield stress); however, this material would not be transferred directly from Tank $41 \mathrm{H}$ to DWPF. The MST/sludge slurry will be transferred from Tank $41 \mathrm{H}$ to Tank $42 \mathrm{H} / 51 \mathrm{H}$ and then on to Tank $40 \mathrm{H}$ as part of a sludge batch. The ground CST will be transferred from the grinder in Tank $41 \mathrm{H}$ to Tank $40 \mathrm{H}$. Tank $40 \mathrm{H}$ is the DWPF feed tank. These yield stress measurements would provide the data needed to size pumps for these transfers. 


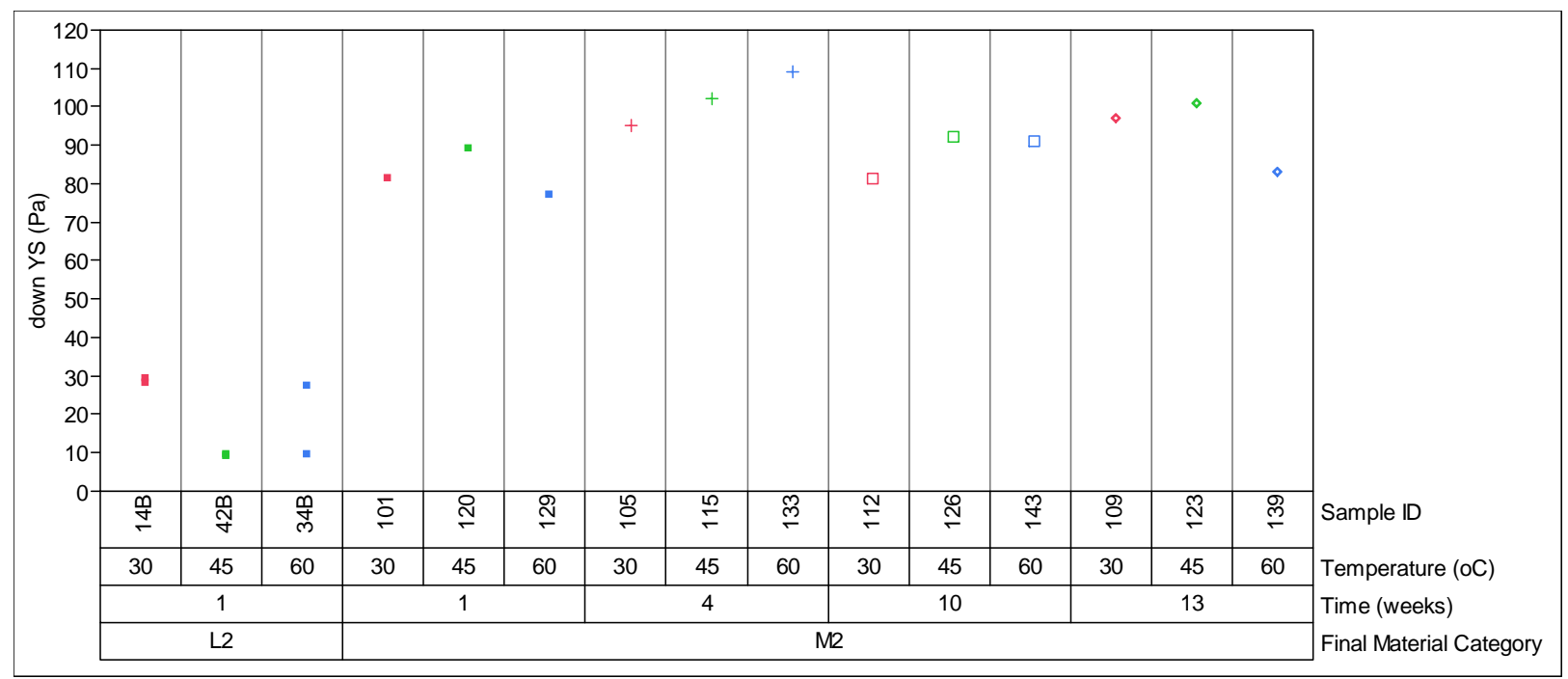

Figure 20. Yield Stress of MST Plus Ground CST Slurries as a Function of Time and Temperature

Figure 21 shows the slurry consistency as a function of settling time and temperature for slurries containing MST and ground CST (1:17 ratio). The plot suggests that settling time and temperature do not have an effect on the slurry consistency. The data also indicate that samples with VSL ground CST (M2) had higher overall consistency than those containing SRNL ground CST (L2); this trend is statistically significant. Slurries with VSL ground CST also had higher shear strengths than equivalent slurries with SRNL ground CST.

Typical consistency measurements of MST plus VSL ground CST range between 45 and 73 cP.

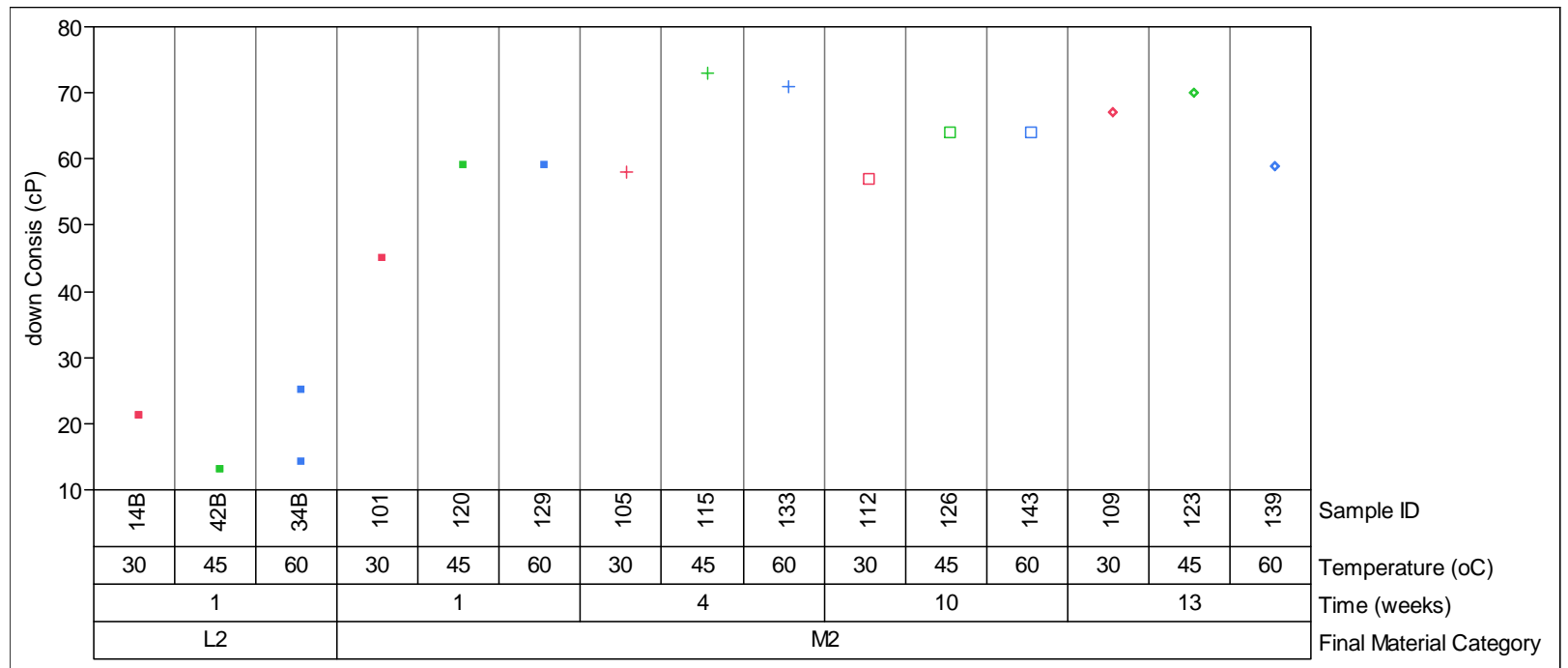

Figure 21. Consistency of MST Plus Ground CST Slurries as a Function of Time and Temperature

Figure 22 shows the slurry yield stress as a function of settling time and temperature for slurries containing MST and unground CST (1:17 ratio). The plot suggests that settling time and 
temperature do not have an effect on the slurry yield stress. Yield stress values for these samples ranged between 37 and $175 \mathrm{~Pa}$, and large variations were observed between measured values for replicates.

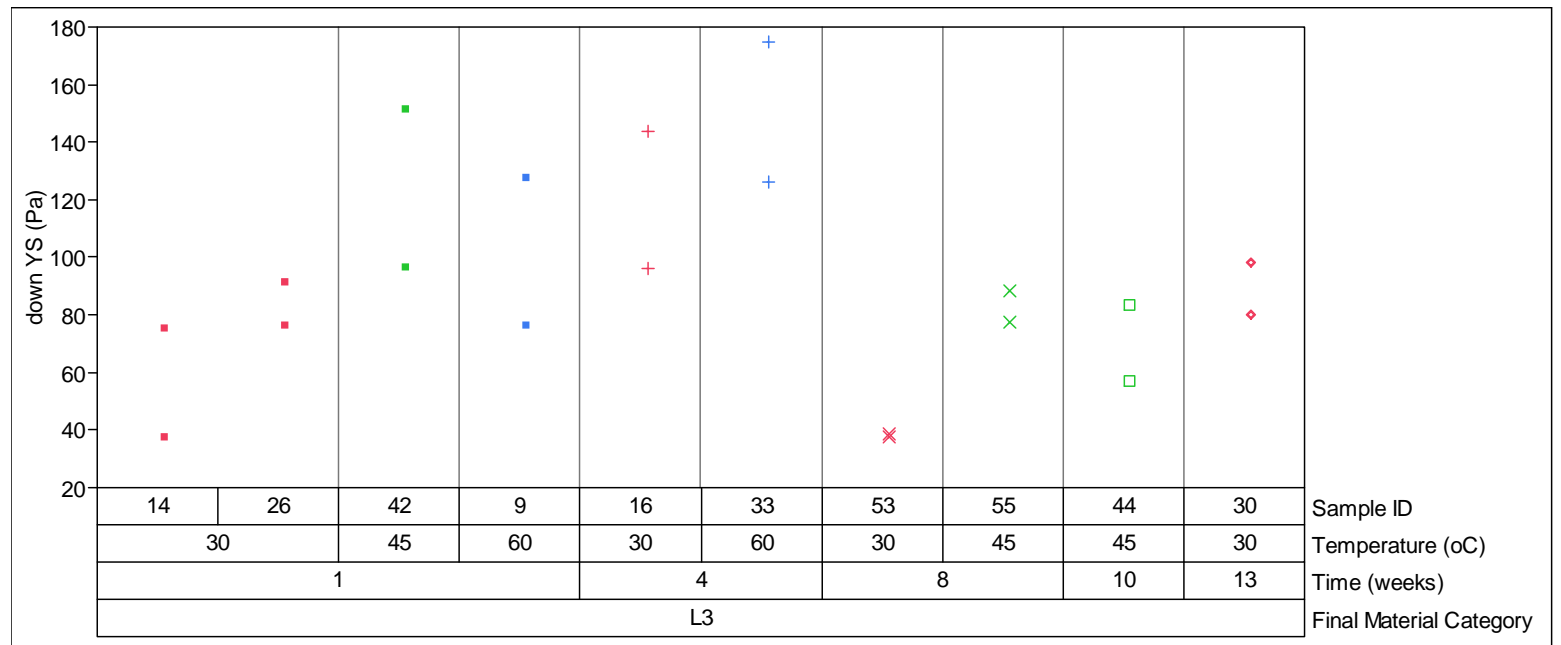

Figure 22. Yield Stress of MST Plus Unground CST Slurries as a Function of Time and Temperature

Figure 23 shows the slurry consistency as a function of settling time and temperature for slurries containing MST and unground CST (1:17 ratio). The plot suggests that settling time and temperature do not have an effect on the slurry consistency.

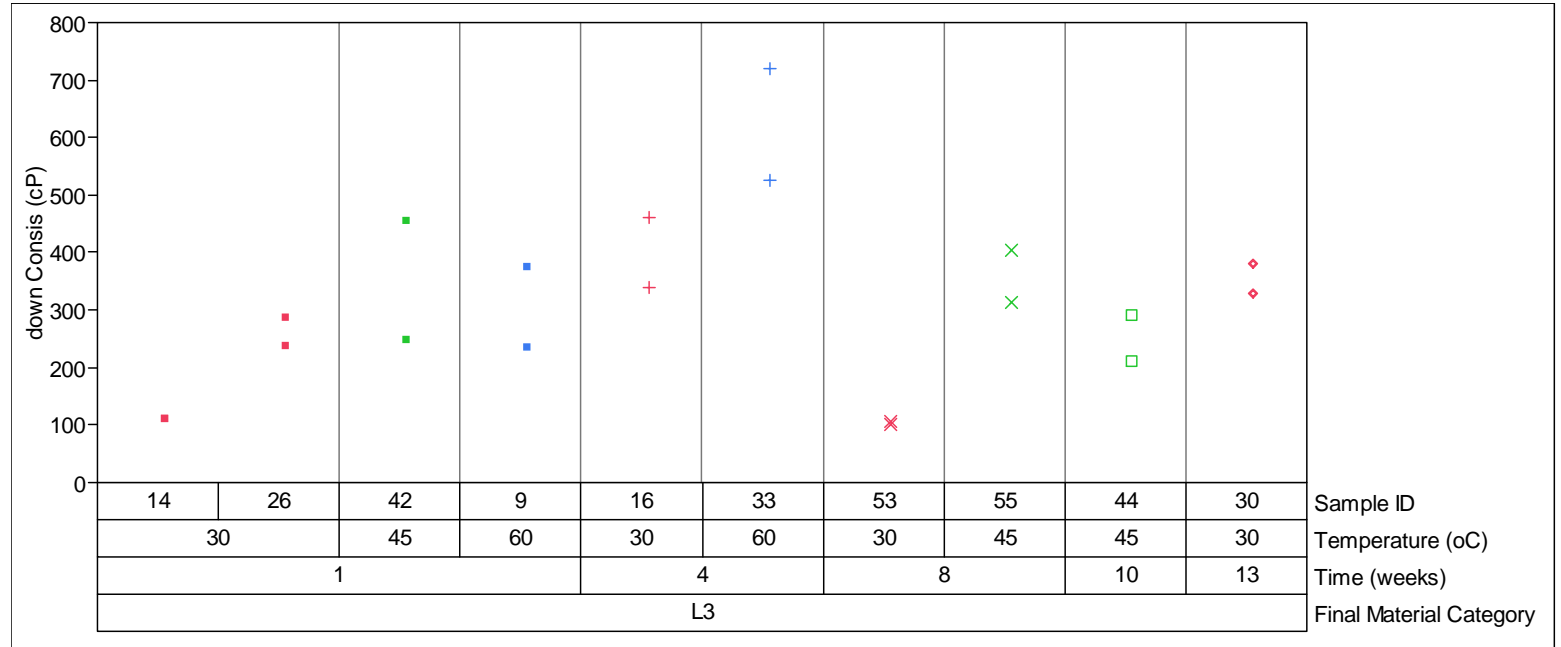

Figure 23. Consistency of MST Plus Unground CST Slurries as a Function of Time and Temperature

Figure 24 shows the slurry yield stress as a function of settling time and temperature for slurries containing MST and sludge (1:1.5 ratio). As mentioned previously, this L4 material is the feed most reflective of the expected material in Tank $41 \mathrm{H}$, which will be transported into Tank $42 \mathrm{H}$ and/or $51 \mathrm{H}$. The plot suggests that settling time and temperature do not have an effect on the slurry yield stress. This result is somewhat surprising. Previous testing has shown that 
sludge/MST slurries that settled for extended times at elevated temperatures exhibit an increase in shear strength and yield stress. ${ }^{2,3}$

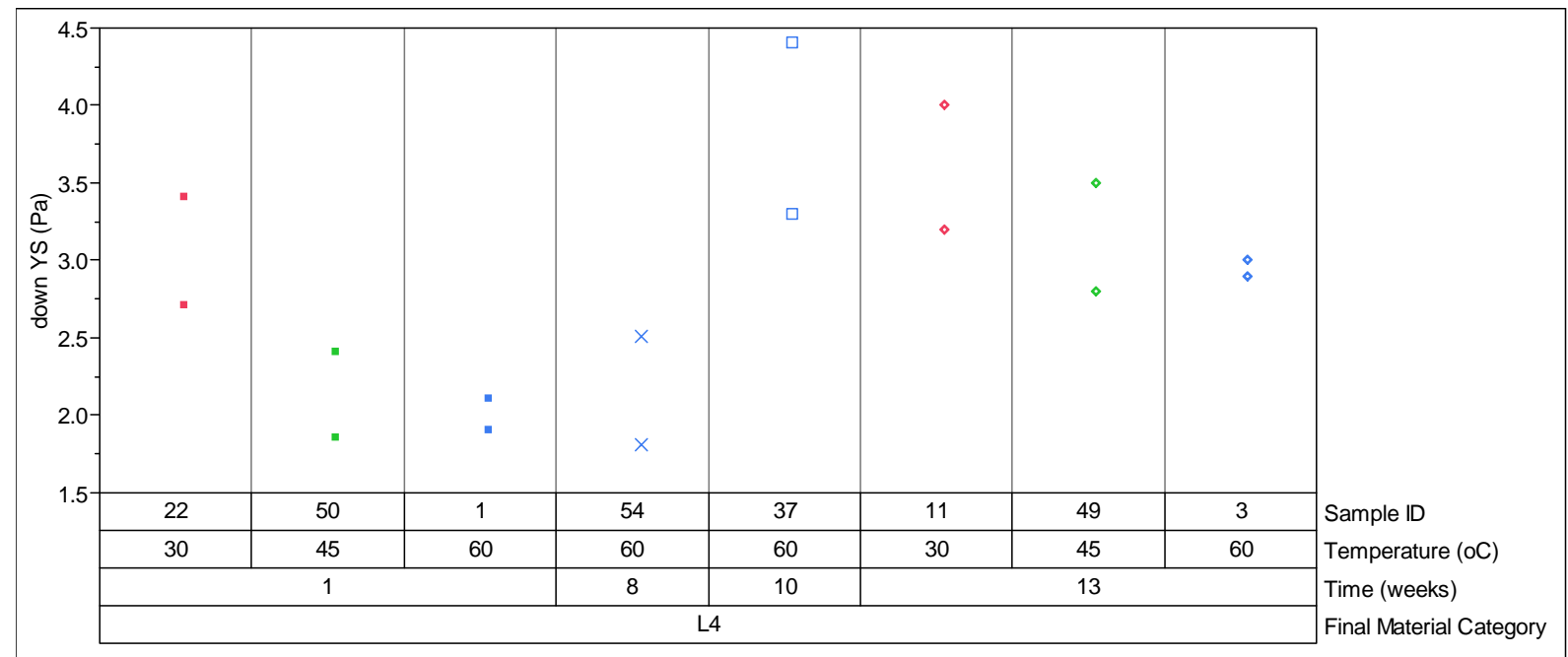

Figure 24. Yield Stress of MST Plus Low Sludge Slurries as a Function of Time and Temperature

Figure 25 shows the slurry consistency as a function of settling time and temperature for slurries containing MST and sludge (1:1.5 ratio). The plot suggests that settling time and temperature do not have any predictable pattern or statistically significant effect on the slurry consistency.

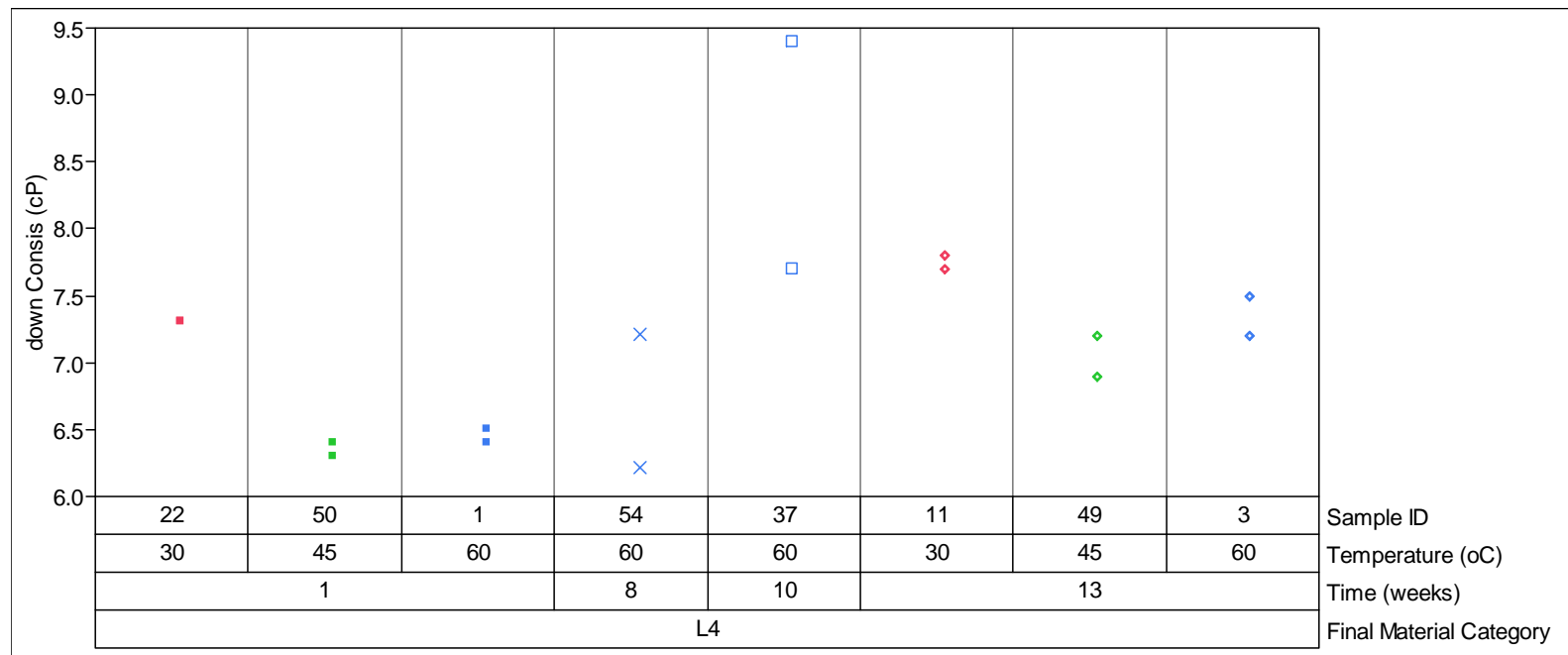

Figure 25. Consistency of MST Plus Low Sludge Slurries as a Function of Time and Temperature

Figure 26 shows the slurry yield stress as a function of settling time and temperature for slurries containing MST, ground CST, and sludge (1:17:660 ratio). The L5/M5 material is reflective of the material in Tank $40 \mathrm{H}$. The plot suggests that settling time and temperature do not have an effect on the slurry yield stress. The yield stress is acceptable for transferring the slurry from $\mathrm{H}$ area to DWPF. In addition, the plot shows no difference in the yield stress of slurries prepared with VSL ground CST compared to slurries prepared with SRNL ground CST. One plausible 
explanation for these results is that the properties of MST and CST components were overwhelmed by the sludge volume in this feed.

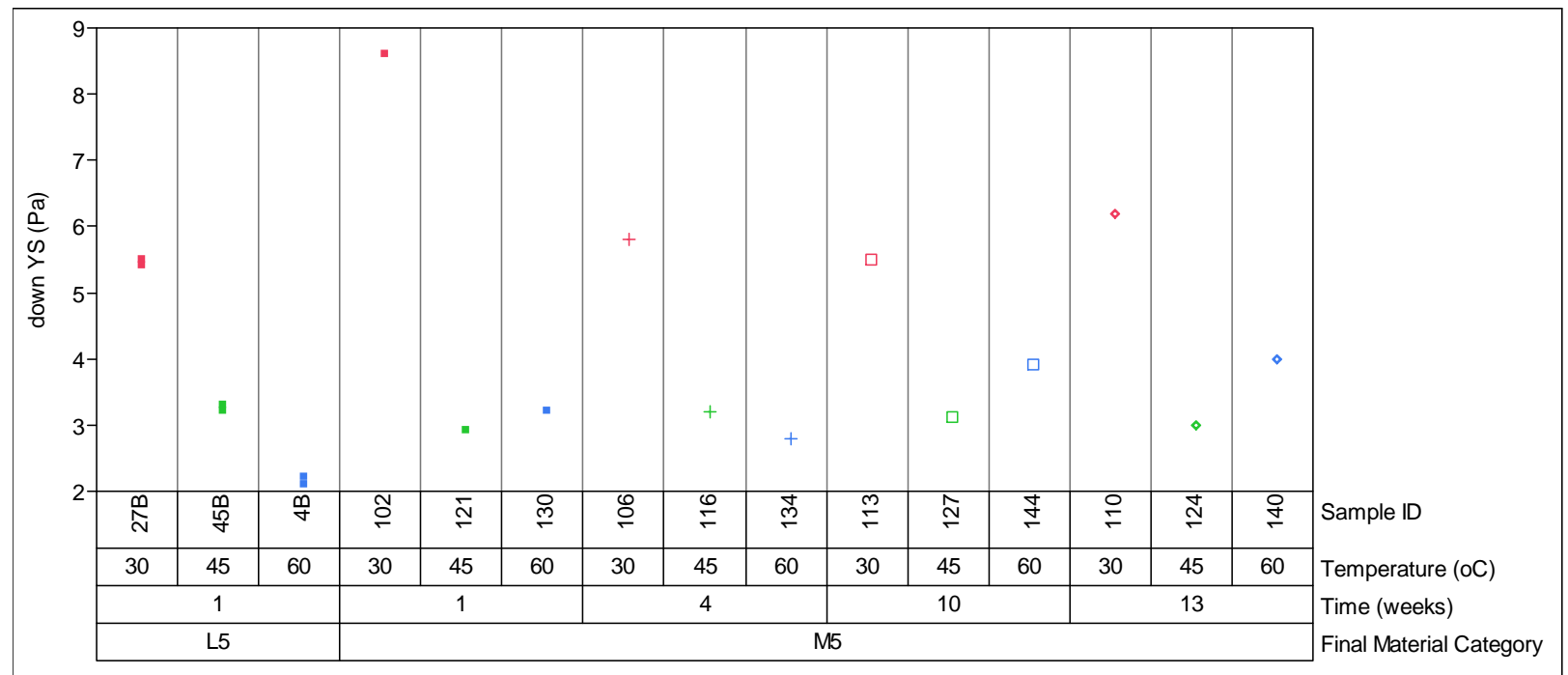

Figure 26. Yield Stress of MST Plus Ground CST Plus High Sludge Slurries as a Function of Time and Temperature

Figure 27 shows the slurry consistency as a function of settling time and temperature for slurries containing MST, ground CST, and sludge (1:17:660 ratio). The plot suggests that settling time and temperature do not have an effect on the slurry consistency. In addition, the plot shows no difference in the consistency of slurries prepared with VSL ground CST compared to slurries prepared with SRNL ground CST. One plausible explanation for these results is that the properties of MST and CST components were overwhelmed by the sludge volume in this feed.

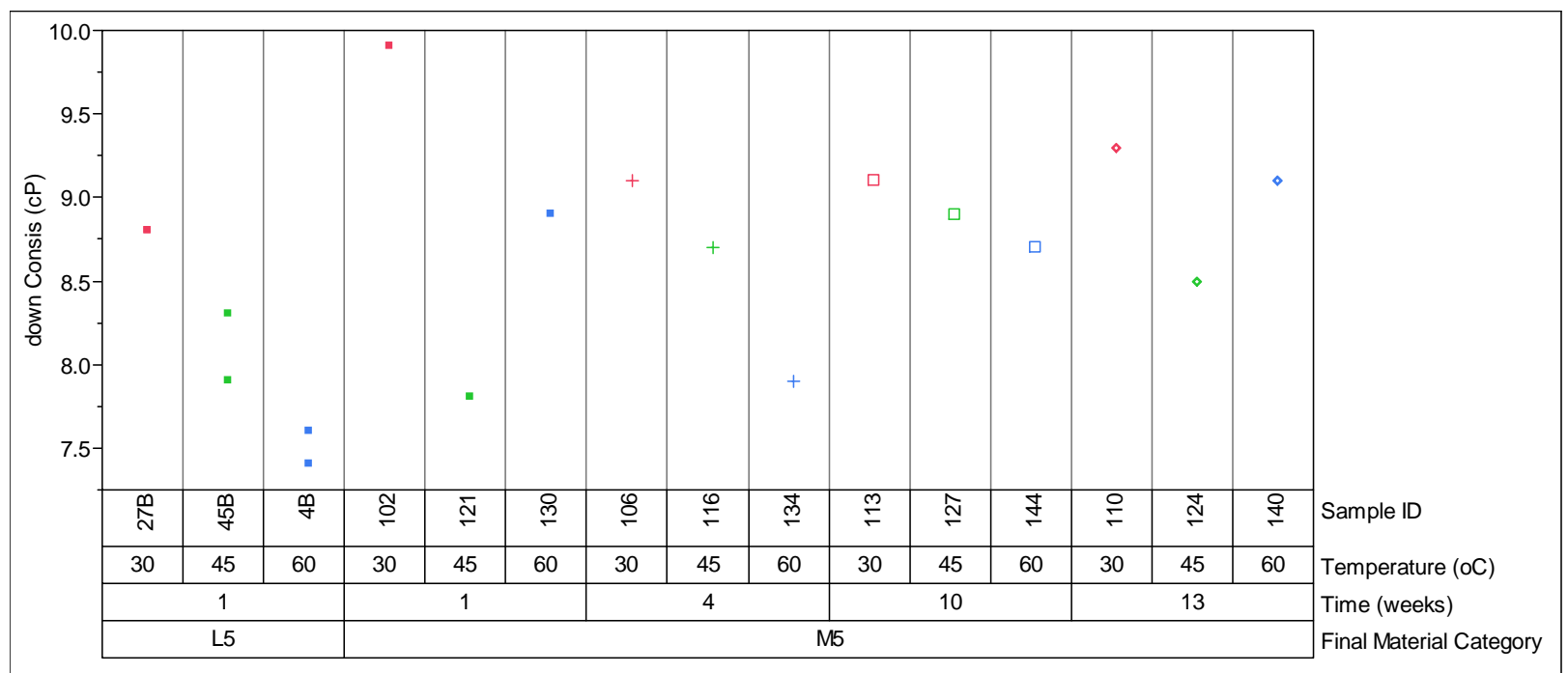

Figure 27. Consistency of MST Plus Ground CST Plus High Sludge Slurries as a Function of Time and Temperature

Figure 28 shows the slurry yield stress as a function of settling time and temperature for slurries containing MST, unground CST, and sludge (1:17:660 ratio). The plot suggests that settling 
time and temperature do not have an effect on the slurry yield stress. As in the previous feeds, the properties of MST and CST components may have been overwhelmed by the sludge volume in this feed. The yield stress meets the acceptance criteria for H-area to DWPF transfers.

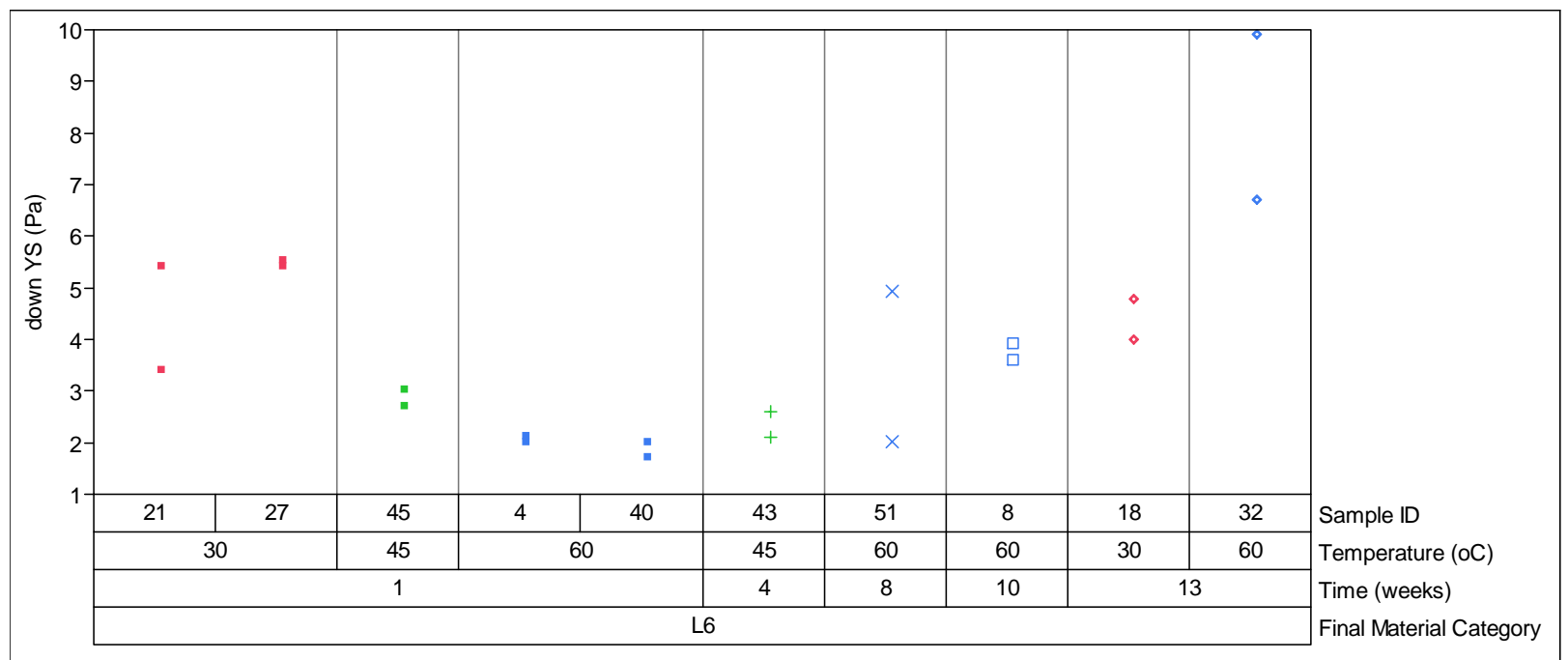

Figure 28. Yield Stress of MST Plus Unground CST Plus High Sludge Slurries as a Function of Time and Temperature

Figure 29 shows the slurry consistency as a function of settling time and temperature for slurries containing MST, unground CST, and sludge (1:17:660 ratio). The plot suggests that settling time and temperature do not have an effect on the slurry consistency. Again, the properties of MST and CST components may have been overwhelmed by the sludge volume in this feed.

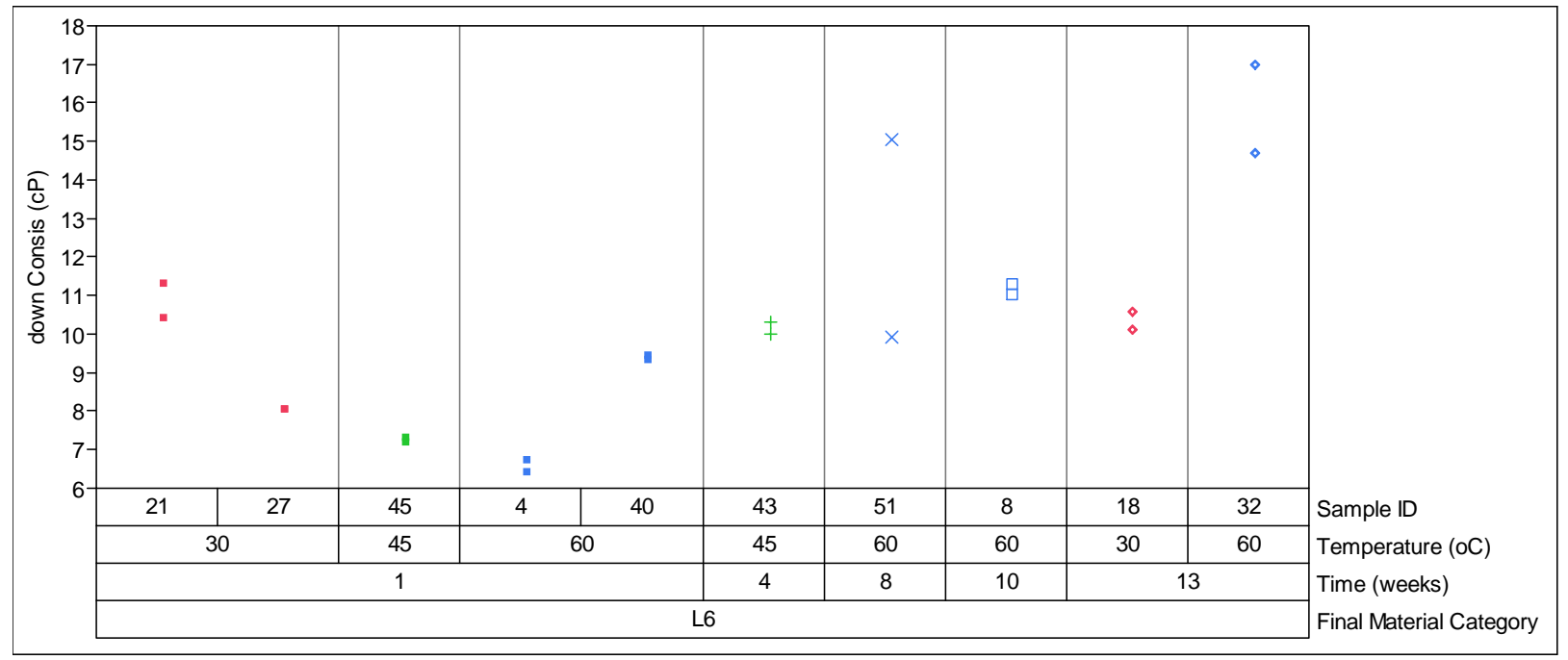

Figure 29. Consistency of MST Plus Unground CST Plus High Sludge Slurries as a Function of Time and Temperature

Figure 30 shows the slurry yield stress as a function of settling time and temperature for slurries containing MST and sludge (1:660 ratio). This is representative of Tank 42H/51H materials. The plot suggests that settling time and temperature do not have an effect on the slurry yield stress. 
This result is somewhat surprising. Previous testing has shown that sludge/MST slurries that settled for extended times at elevated temperatures exhibit an increase in shear strength and yield stress. ${ }^{2,3}$ However, this slurry contains mostly sludge, while the slurries in the previous tests contained approximately equal amounts of sludge and MST. Given the large fraction of sludge, this slurry likely exhibits more of the characteristics of sludge than MST. The yield stress of this material would not present any problems for transfer from Tank $42 \mathrm{H}$ or Tank $51 \mathrm{H}$ to Tank $40 \mathrm{H}$.

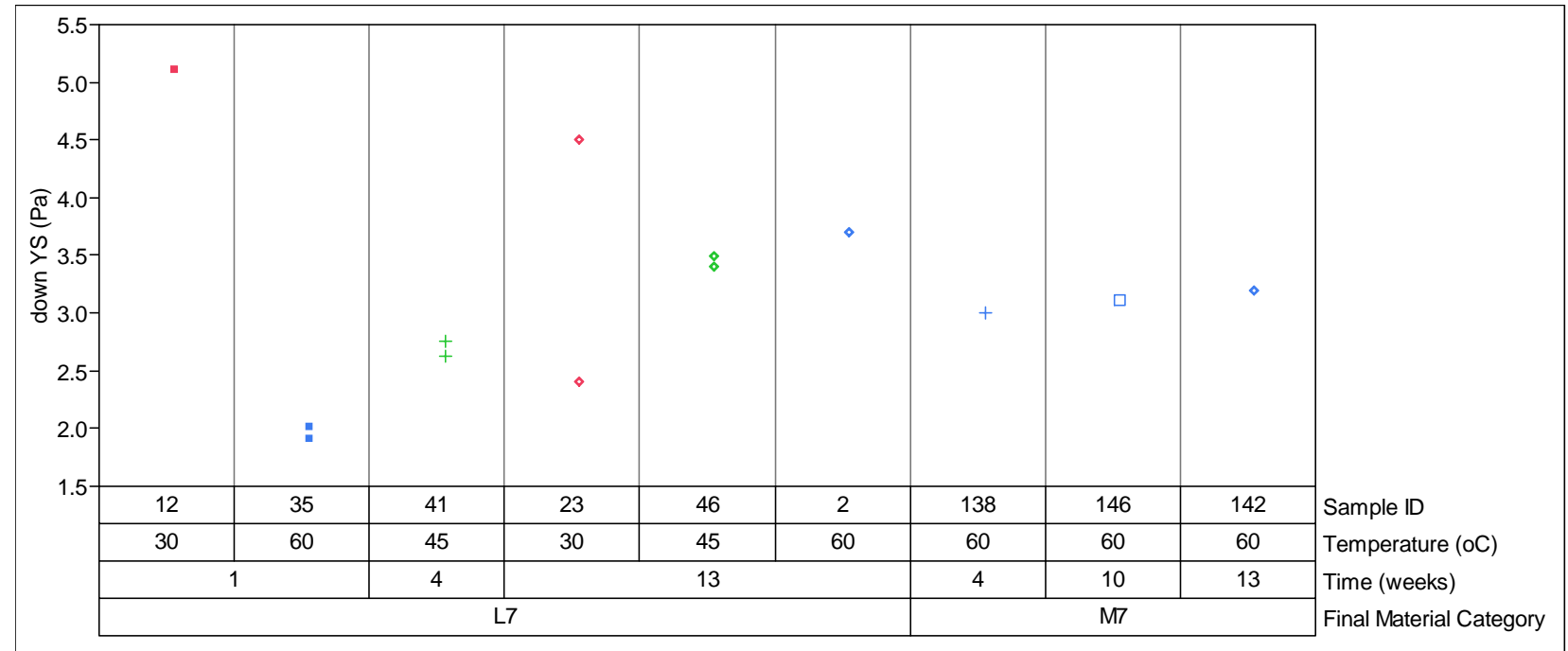

Figure 30. Yield Stress of MST Plus High Sludge Slurries as a Function of Time and Temperature

Figure 31 shows the slurry consistency as a function of settling time and temperature for slurries containing MST and sludge (1:660 ratio). The plot suggests that settling time and temperature do not have an effect on the slurry consistency.

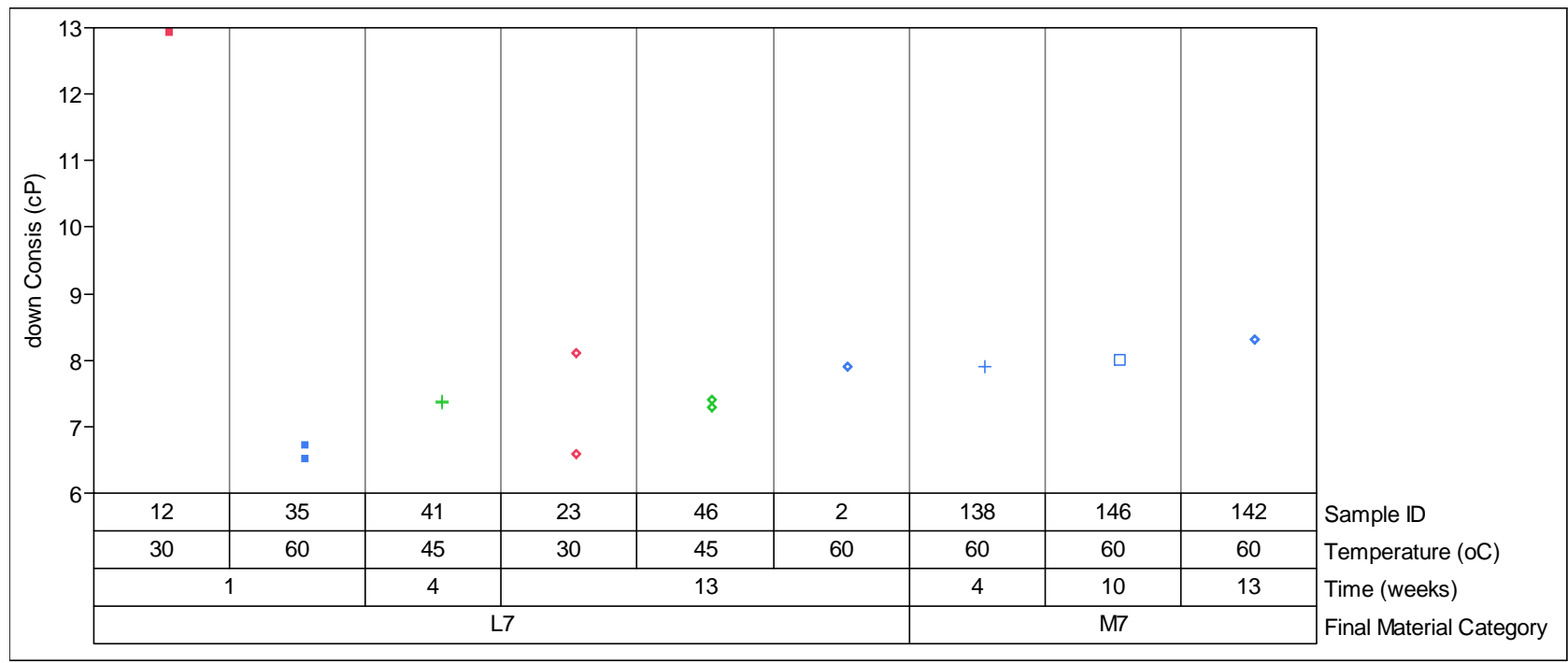

Figure 31. Consistency of MST Plus High Sludge Slurries as a Function of Time and Temperature 
Figure 32 shows the slurry yield stress as a function of settling time and temperature for slurries containing MST, ground CST, and sludge (1:17:1.5 ratio). The plot suggests that settling time and temperature do not have an effect on the slurry yield stress. In addition, the plot compares slurries with CST ground by VSL (M8) with slurries ground by SRNL (L8). The slurries containing VSL ground CST had a higher yield stress than the slurries containing SRNL ground VSL.

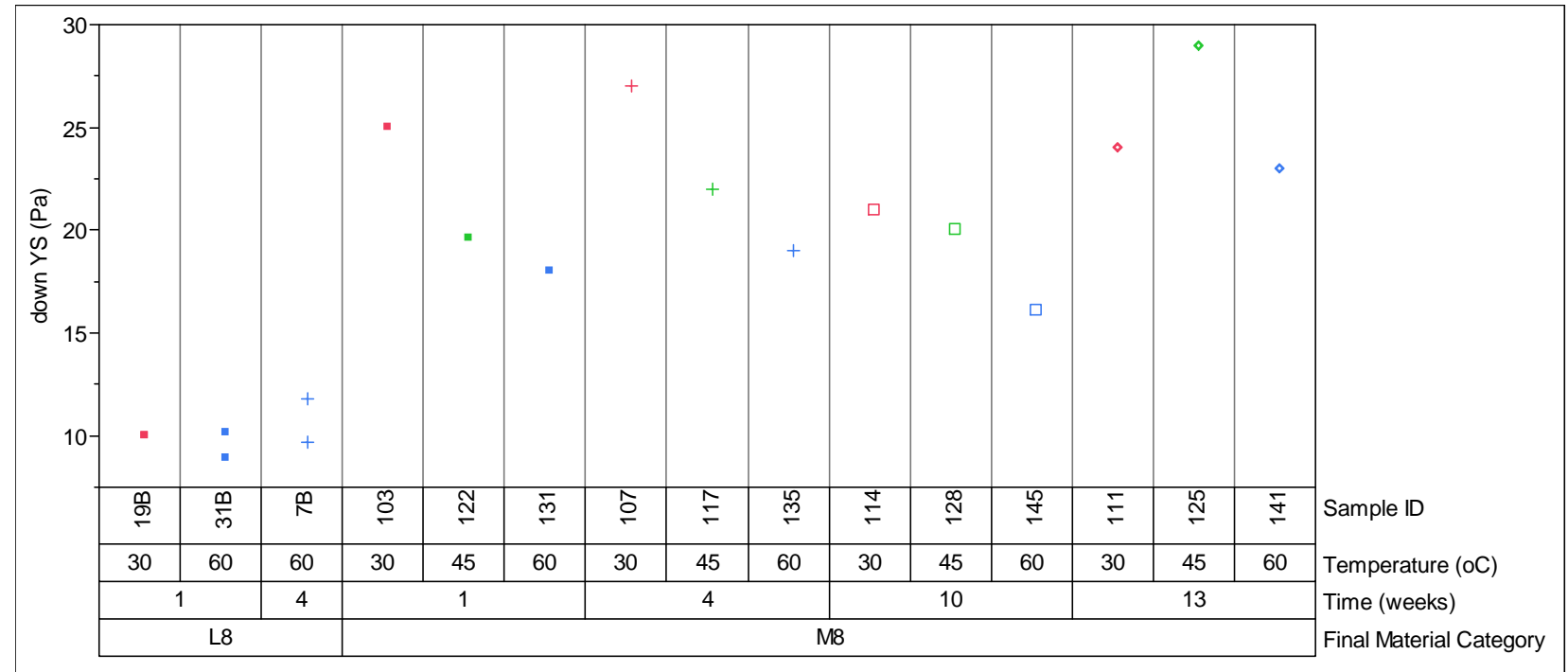

Figure 32. Yield Stress of MST Plus Ground CST Plus Low Sludge Slurries as a Function of Time and Temperature

Figure 33 shows the slurry consistency as a function of settling time and temperature for slurries containing MST, ground CST, and sludge (1:17:1.5 ratio). The plot suggests that settling time and temperature do not have an effect on the slurry consistency. In addition, the plot compares slurries with CST ground by VSL (M8) with slurries ground by SRNL (L8). The slurries containing VSL ground CST had a higher consistency than the slurries containing SRNL ground VSL. 


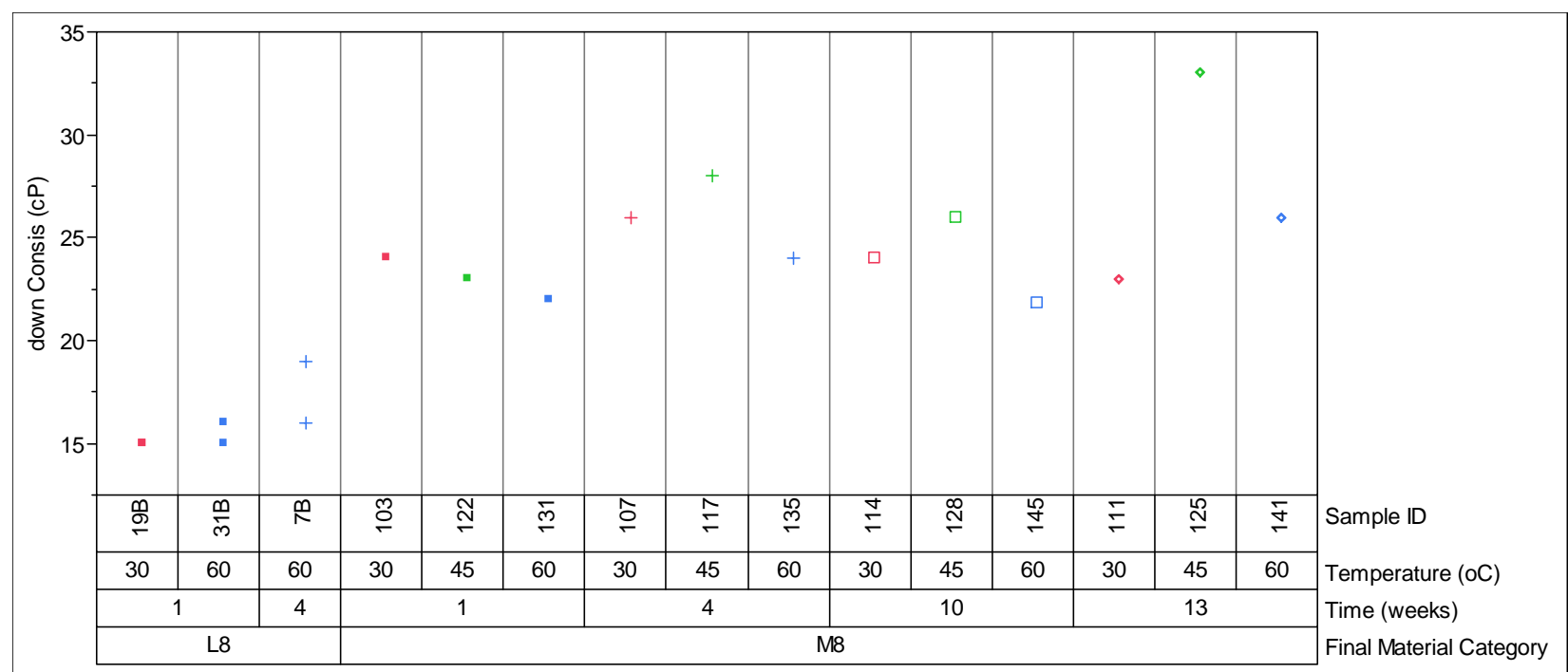

Figure 33. Consistency of MST Plus Ground CST Plus Low Sludge Slurries as a Function of Time and Temperature

Figure 34 shows the slurry yield stress as a function of settling time and temperature for slurries containing MST, unground CST, and sludge (1:17:1.5 ratio). The plot suggests that settling time and temperature do not have an effect on the slurry yield stress.

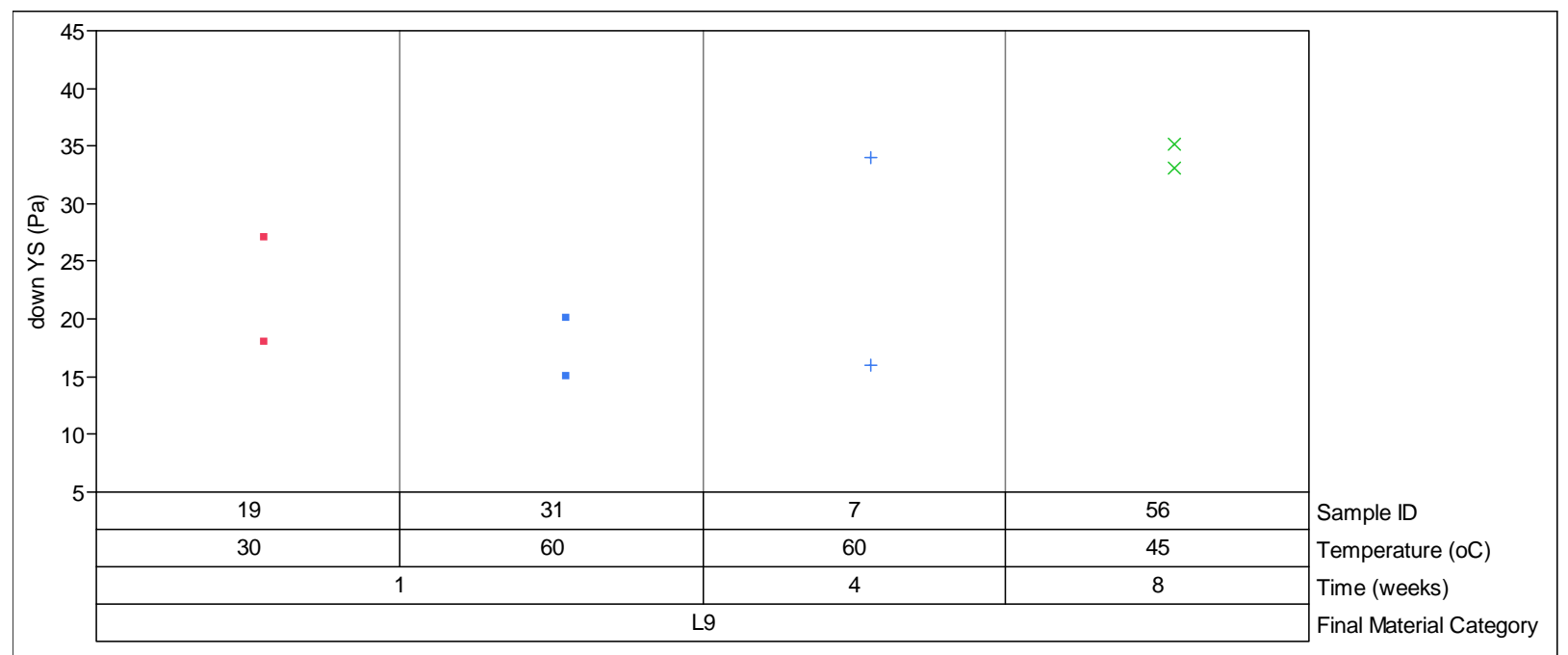

Figure 34. Yield Stress of MST Plus Unground CST Plus Low Sludge Slurries as a Function of Time and Temperature

Figure 35 shows the slurry consistency as a function of settling time and temperature for slurries containing MST, unground CST, and sludge (1:17:1.5 ratio). The plot suggests that settling time and temperature do not have an effect on the slurry consistency. 


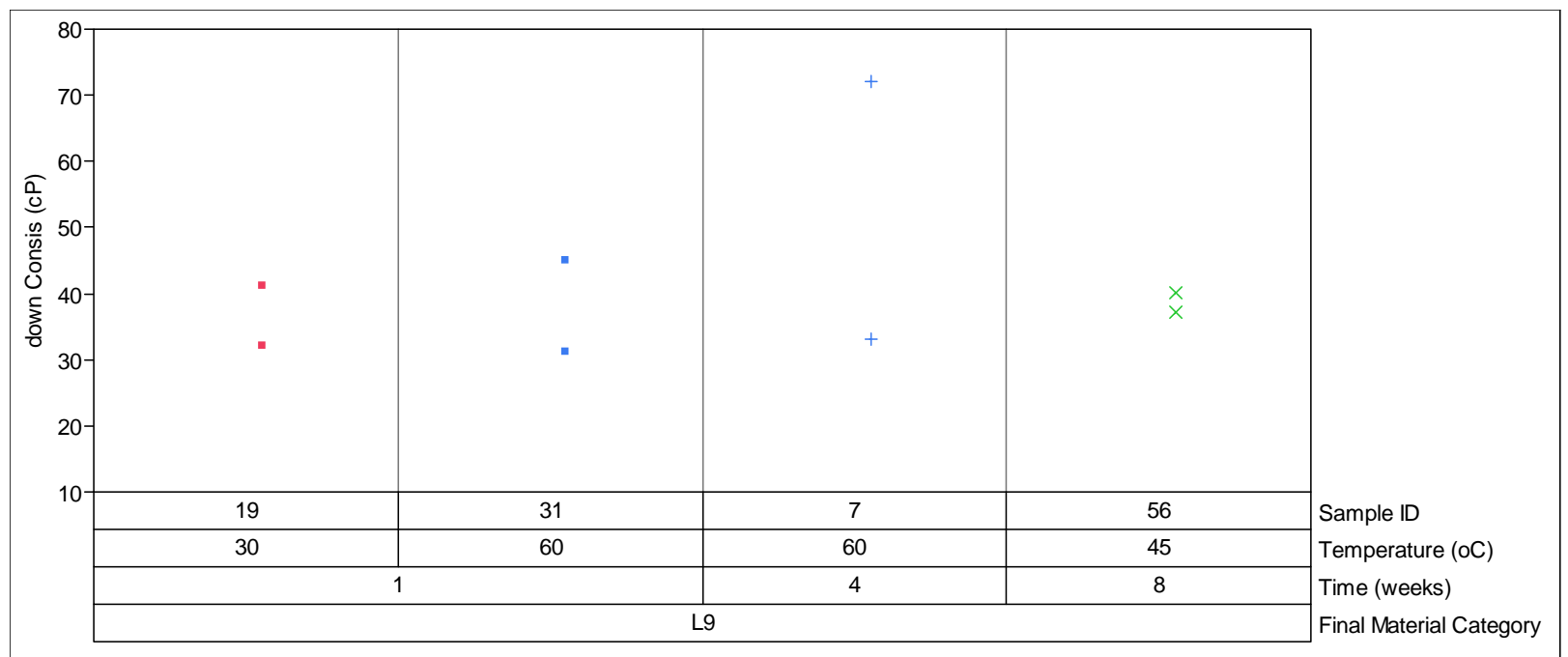

Figure 35. Consistency of MST Plus Unground CST Plus Low Sludge Slurries as a Function of Time and Temperature

Figure 36 shows the slurry yield stress as a function of settling time and temperature for slurries containing MST, ground CST, and sludge (1:17:82 ratio). The plot suggests that settling time and temperature do not have an effect on the slurry yield stress.

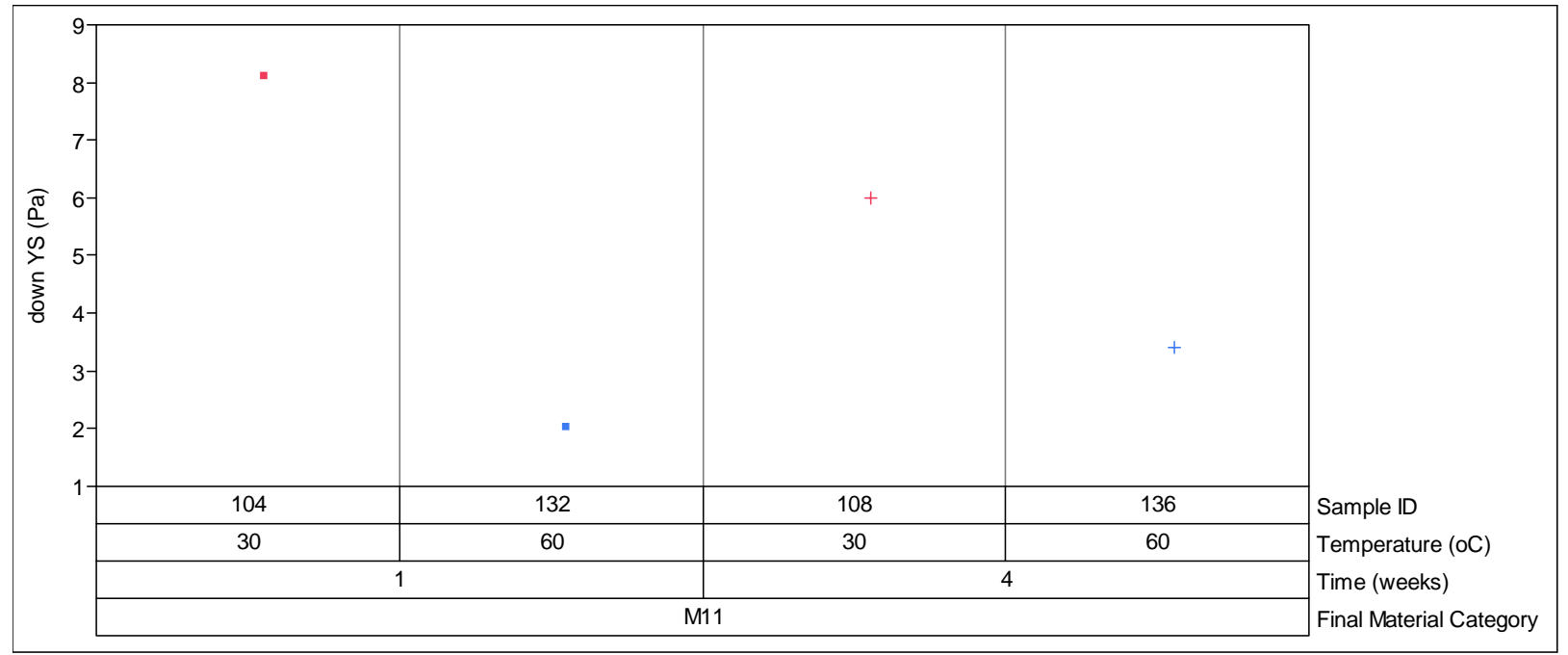

Figure 36. Yield Stress of MST Plus Ground CST Plus Medium Sludge Slurries as a Function of Time and Temperature

Figure 37 shows the slurry consistency as a function of settling time and temperature for slurries containing MST, ground CST, and sludge (1:17:82 ratio). The plot suggests that settling time and temperature do not have an effect on the slurry consistency. 


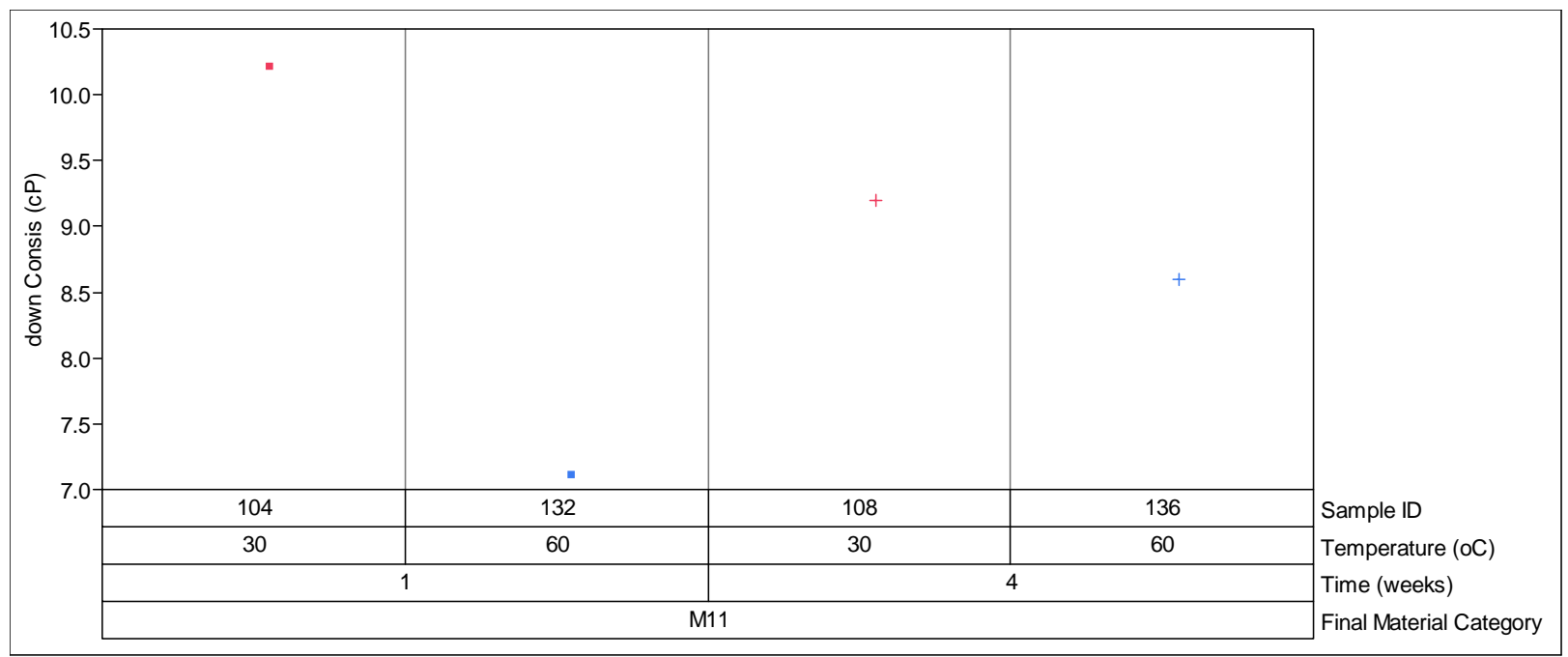

Figure 37. Consistency of MST Plus Ground CST Plus Medium Sludge Slurries as a Function of Time and Temperature

Feed M10 is not discussed herein because samples tested provided insufficient data points for statistical analysis or meaningful plot depiction.

Feed M12 is not discussed herein because samples tested provided insufficient data points for statistical analysis or meaningful plot depiction.

The yield stress and consistency measurements did not show as strong of a correlation with settling time and temperature as the shear strength measurements. One likely reason for this difference is the samples were mixed prior to performing the yield stress and consistency measurements. The mixing will break some of the bonds that have formed between particles and reduce the effects of settling time and temperature on slurry rheology. Because the samples are not mixed prior to measuring the shear strength, the measured shear strength showed a stronger correlation with settling time and temperature.

\subsection{IRRADIATION TESTS}

Table 6 shows the rheological results from the samples that were exposed to radiation levels of 0 , 10, and $100 \mathrm{MRad}$. Because of the large variance between replicate samples, the control samples are being repeated, and the results will be documented further in a later report. Sample 5-2 was determined to be an outlier, due to evaporation that occurred during the 4 week settling time. Treatment of the sample was not repeated, due to the time required to complete the irradiation process and the unavailability of the Co-60 irradiation source.

Measurements obtained from samples 1, 3, and 5 are representative of M8 or "low sludge” feeds. The large variances between replicates in samples 1 and 5 make interpretation of the data more difficult. Process knowledge and comparison with samples from the extended matrix suggest that shear strengths observed in sample 1-1 may be an outlier. Samples 1, 3 and 5 do not show increases in the shear strength, yield stress, or consistency as the dose is increased from 0 to 100 MRad. 
Measurements obtained from samples 2, 4, and 6 are representative of M5 or "high sludge" feeds. Replicate samples showed some variance, but values for all measurements corresponded well with samples from the extended matrix. Samples 2, 4 and 6 do not show increases in the shear strength, yield stress, or consistency as the dose is increased from 0 to 100 MRad.

Table 6. Irradiation Test Results

\begin{tabular}{|c|c|c|c|c|c|}
\hline Sample & Temperature $\left({ }^{\circ} \mathrm{C}\right)$ & Dose & Shear Strength & Yield Stress & Consistency \\
\hline $1-1$ & 60 & Control & $985.8 \mathrm{~Pa}$ & \multirow{2}{*}{$9.9 \mathrm{~Pa}$} & \multirow{2}{*}{$14.0 \mathrm{cP}$} \\
\hline $1-2$ & 60 & Control & $61 \mathrm{~Pa}$ & & \\
\hline $2-1$ & 60 & Control & $44.2 \mathrm{~Pa}$ & \multirow{2}{*}{$0.7 \mathrm{~Pa}$} & \multirow{2}{*}{$4.2 \mathrm{cP}$} \\
\hline $2-2$ & 60 & Control & $63.96 \mathrm{~Pa}$ & & \\
\hline $3-1$ & 60 & $10 \mathrm{MRad}$ & $38.98 \mathrm{~Pa}$ & \multirow{2}{*}{$6.1 \mathrm{~Pa}$} & \multirow{2}{*}{$11.1 \mathrm{cP}$} \\
\hline $3-2$ & 60 & 10 MRad & $75.3 \mathrm{~Pa}$ & & \\
\hline $4-1$ & 60 & 10 MRad & $28.97 \mathrm{~Pa}$ & \multirow{2}{*}{$3.1 \mathrm{~Pa}$} & \multirow{2}{*}{$7.0 \mathrm{cP}$} \\
\hline $4-2$ & 60 & 10 MRad & $13.01 \mathrm{~Pa}$ & & \\
\hline $5-1$ & 60 & 100 MRad & $96.1 \mathrm{~Pa}$ & \multirow{2}{*}{$0.9 \mathrm{~Pa}$} & \multirow{2}{*}{$4.1 \mathrm{cP}$} \\
\hline $5-2$ & 60 & 100 MRad & $552.7 \mathrm{~Pa}$ & & \\
\hline $6-1$ & 60 & 100 MRad & $29.84 \mathrm{~Pa}$ & \multirow{2}{*}{$0.7 \mathrm{~Pa}$} & \multirow{2}{*}{$4.0 \mathrm{cP}$} \\
\hline $6-2$ & 60 & 100 MRad & $28.7 \mathrm{~Pa}$ & & \\
\hline
\end{tabular}

\subsection{OBSERVATIONS}

Various gross observations were noted as samples were monitored during time and temperature treatments. During sample preparation, bottle contents were mixed by hand to homogeneity prior to initiation of settling and controlled temperatures, as shown in Figure 38. In nearly all samples, salt solution supernate quickly formed a surface layer over the sample, indicating that solid materials were beginning to settle (Figure 39a). Despite the uniform volume of salt solutions applied to each sample, observed supernate layers in MST only and MST plus CST samples were markedly less than other samples, indicating that the supernate was largely held within the solids matrix.

In samples held at elevated temperatures $\left(45^{\circ} \mathrm{C}\right.$ or greater $)$, stratification of sample components was found after only a few weeks of settling. Low and high sludge samples were noted to have a distinct layer of darker material, $\sim 1 \mathrm{~cm}$ in depth, near the surface of the solid content of the sample, as seen in Figure 39b. Many settled solid layers in the sludge-rich samples were also observed to have light to white colored solid masses on the bottom that had very little of the brown color associated with the sludge solids. These results suggest that the lighter colored components of the feed (MST and CST) somehow disengaged from the darker solids of the sludge.

Similarly, clumps of solids up to $1 \mathrm{~cm}$ in diameter were found suspended within samples treated at various temperatures and settling times. Clumps were often lighter than the surrounding material, and analytical data (i.e., X-ray Diffraction (XRD)) indicate that they were primarily CST. Agglomerations of brown sludge solids were also noted in the body of the sample (Figure 39c,d). 
Samples held at elevated temperatures were observed to slowly release bubbles of entrained air from the solids matrix, forming grooves and channels within the body of solids that were observable through the clear plastic exterior of the bottle. Samples with high sludge content were less prone to display these structures, presumably because sludge solids were able to fill these voids as they settled (Figure 39e).

Formation of loose, fluffy solids within the salt supernate layer was observed in most samples with sludge content. Similarly, sludge-containing samples were frequently observed to have crystalline formations floating on the supernate surface or forming a ring around the sample bottle. These formations were found in samples held for various times at various temperatures, but were notably larger and more prevalent at higher temperatures (Figure 39f).

Figure 40 provides pre- and post-treatment images of selected samples. Further investigation into the causes behind formation of the structures noted may provide additional insight into material properties of MST, CST and sludge feeds.

The segregation of solid particles that was observed in the rheology samples was not observed in the pilot-scale resuspension tests or the tall column settling test.

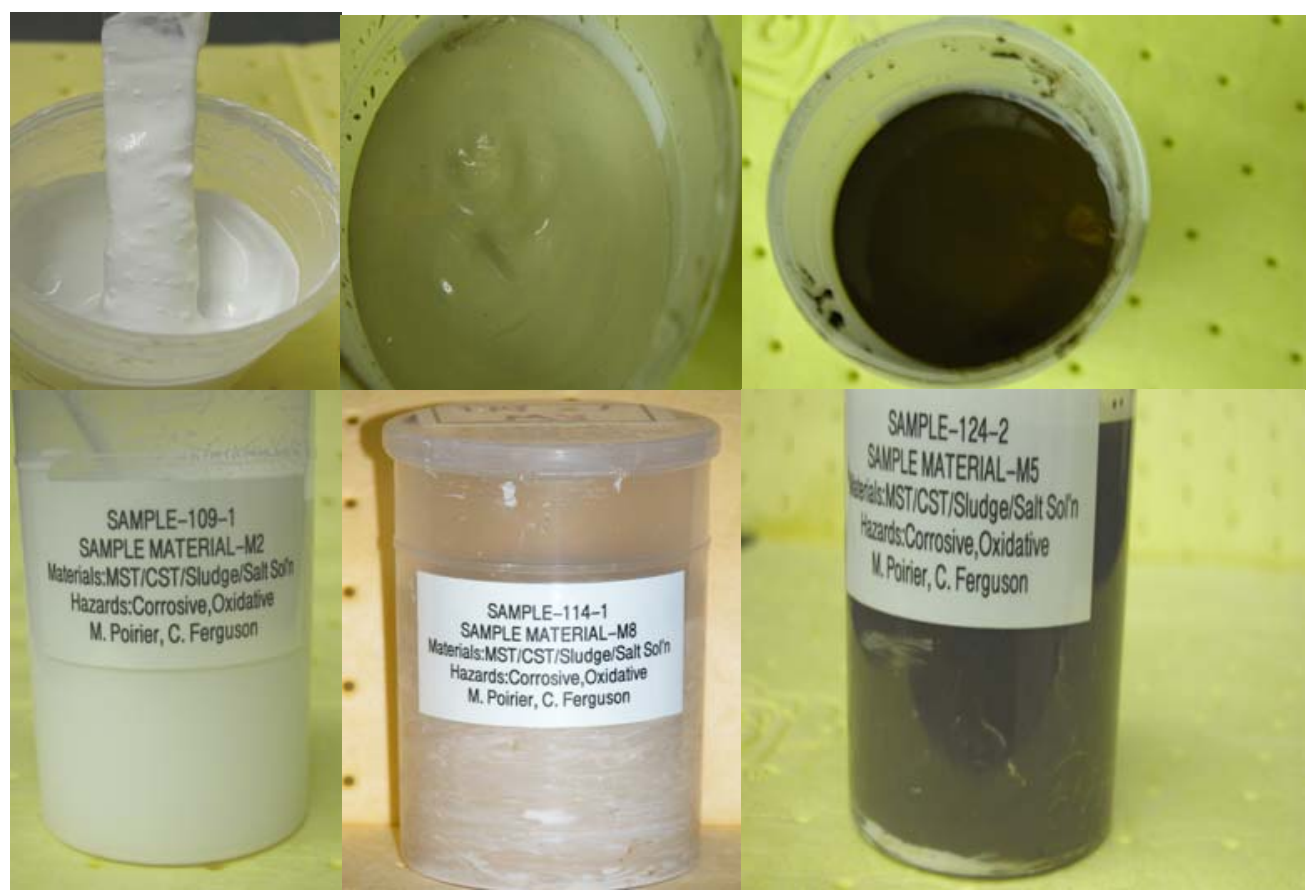

Figure 38. Rheology Samples before Settling 


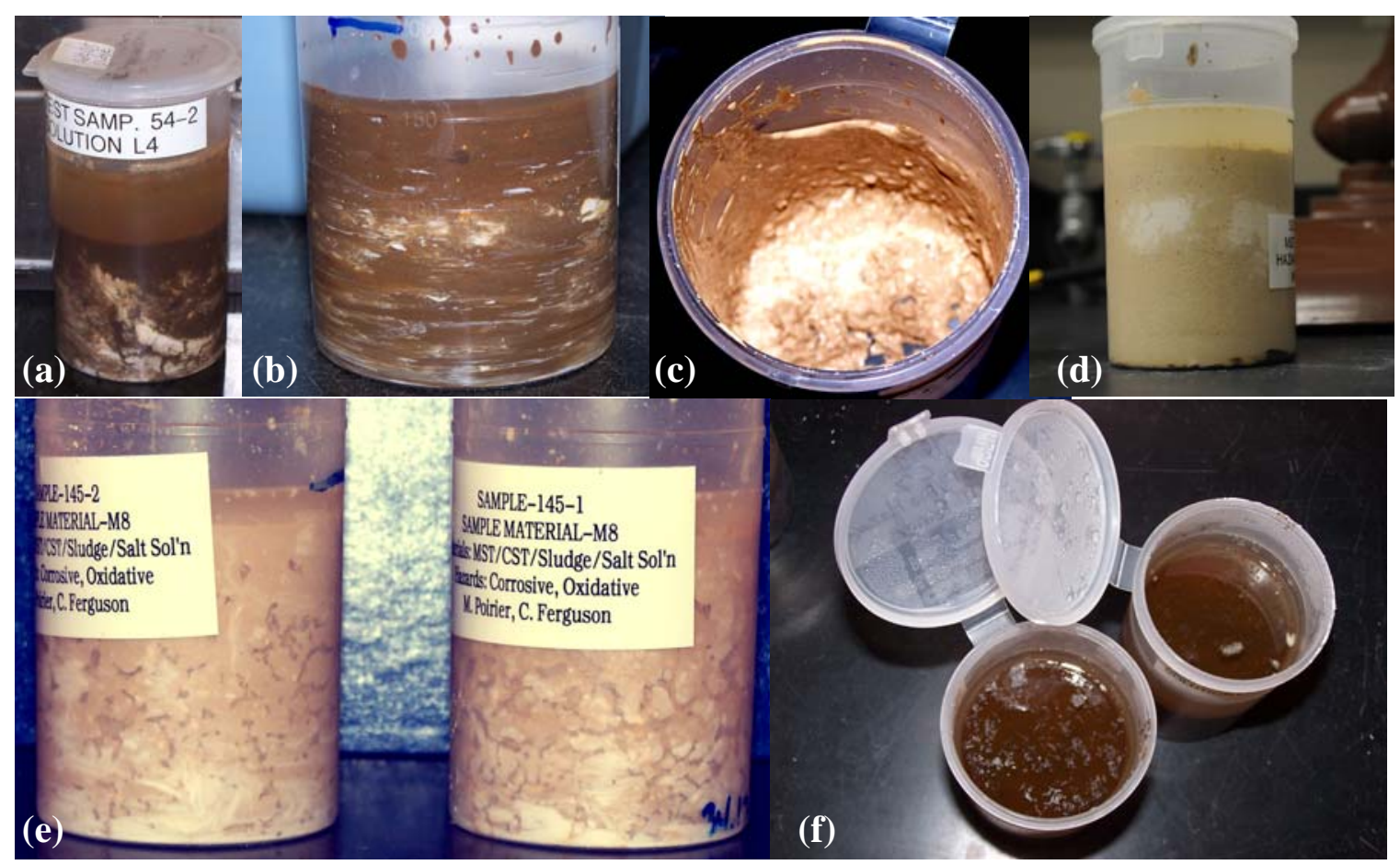

Figure 39. Rheology Samples after settling, showing (a) settled layers of solids and supernate, (b) stratification, (c) and (d) clumping, (e) channels from released air, and (f) "fluffy" solids at the surface of samples.

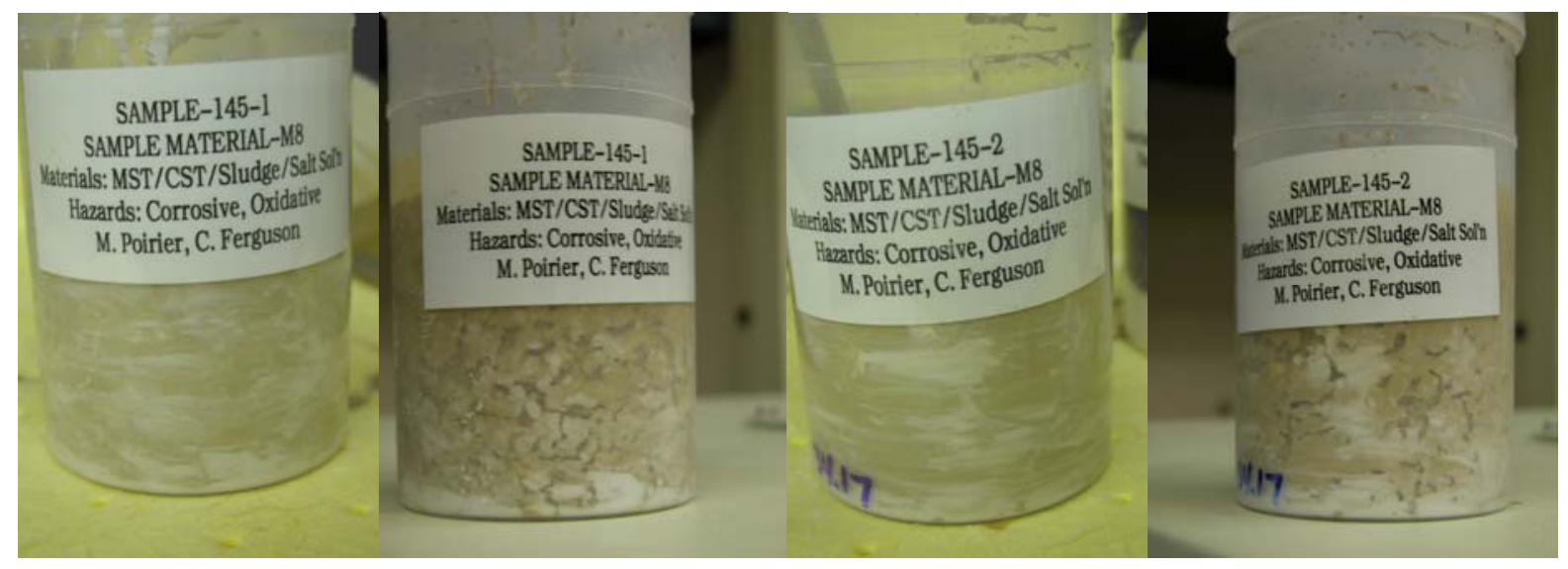

Figure 40. Comparison of rheology sample 145 duplicates after 10 weeks of settling at $60^{\circ} \mathrm{C}$.

\subsection{CONCLUSIONS}

The conclusions from this analysis follow:

- Slurries containing MST and unground CST have the largest shear strength. Due to the high shear strengths measured in slurries containing unground CST, evaluations of specific tank contents and mixing capability should be performed prior to any addition of 
this material into a waste tank. Experimentally determined shear strengths indicate mixing could be problematic in mixtures containing unground CST.

- Increasing the ground CST fraction in the slurry increases the slurry shear strength, yield stress, and consistency.

- Increasing the sludge fraction in the slurry decreases the slurry shear strength, yield stress, and consistency.

- Slurries containing VSL ground CST have larger shear strength, yield stress, and consistency than slurries containing SRNL ground CST.

- The effects of settling time and temperature on slurry shear strength are slurry dependent.

- No effects of settling time and temperature on slurry yield stress or consistency were observed.

- Radiation up to 100 MRad does not appear to affect properties of shear strength, yield stress, and consistency of process feeds. 


\subsection{REFERENCES}

${ }^{1}$ K.M.L. Taylor-Pashow, M. R. Poirier, F. F. Fondeur, T.B. Peters, D.T. Hobbs, and S.D. Fink, “Task Technical and Quality Assurance Plan for Testing to Support Modular Salt Processing Project Monosodium Titanate Studies”, SRNL-RP-2010-00686.

${ }^{2}$ Poirier, M. R.; Herman, D. T.; Fondeur, F. F.; Hansen, E.; and Fink, S. D. "MST/Sludge Agitation Studies for Actinide Removal Process and DWPF”, WSRC TR-2003-00471, October 10, 2003.

${ }^{3}$ Taylor, P. A. and Mattus, C. H., "Resuspension and Settling of Monosodium Titanate and Sludge in Supernate Simulant for the Savannah River Site”, ORNL/TM-1999/166, September 1999.

${ }^{4}$ M. R. Poirier, C. E. Ferguson, and D. C. Koopman, "Rheology of Settled Solids in the Small Column Ion Exchange (SCIX) Process”, SRNL-STI-2011-00054, January 27, 2011.

${ }^{5}$ Herman, D. T. and Poirier, M. R., "Recipe for Simulated Sludge Batch 6-DS for Rotary Filter Testing”, SRNL-TR2009-00111, Rev. 1, October 2009.

${ }^{6}$ D. F. Bickford, G. D. Creech, and D. C. Beam, "Confirmation of Small Column Ion Exchange Crystalline Silicotitanate (CST) Grinder Configuration and Estimation of Treatment Cycle”, WSRC-TR-2005-00282, March 25, 2005.

${ }^{7}$ W. Govier and K. Aziz, The Flow of Complex Mixtures in Pipes. Malabar, FL: Krieger Publishing, 1982.

${ }^{8}$ D. C. Koopman, Rheology Protocols for DWPF Samples, WSRC-RP-2004-00470, October, 2004, Savannah River Site, Aiken, SC, 29808.

${ }^{9}$ JMP version 7.2.2, SAS Institute, Inc., Cary NC, 1989-2007.

${ }^{10}$ M. R. Poirier, Z. H. Qureshi, M. L. Restivo, T. J. Steeper, M. R. Williams, "Investigating Suspension of MST Slurries in a Pilot-Scale Waste Tank”, SRNL-STI-2010-00793, January 24, 2011.

${ }^{11}$ M. R. Poirier, Z. H. Qureshi, M. L. Restivo, T. J. Steeper, M. R. Williams, “Investigating Suspension of MST, CST, and Simulated Sludge Slurries in a Pilot-Scale Waste Tank”, SRNL-STI-2011-00250, April 25, 2011 


\section{APPENDIX 1}

PARTICLE SIZE DISTRIBUTION CURVES

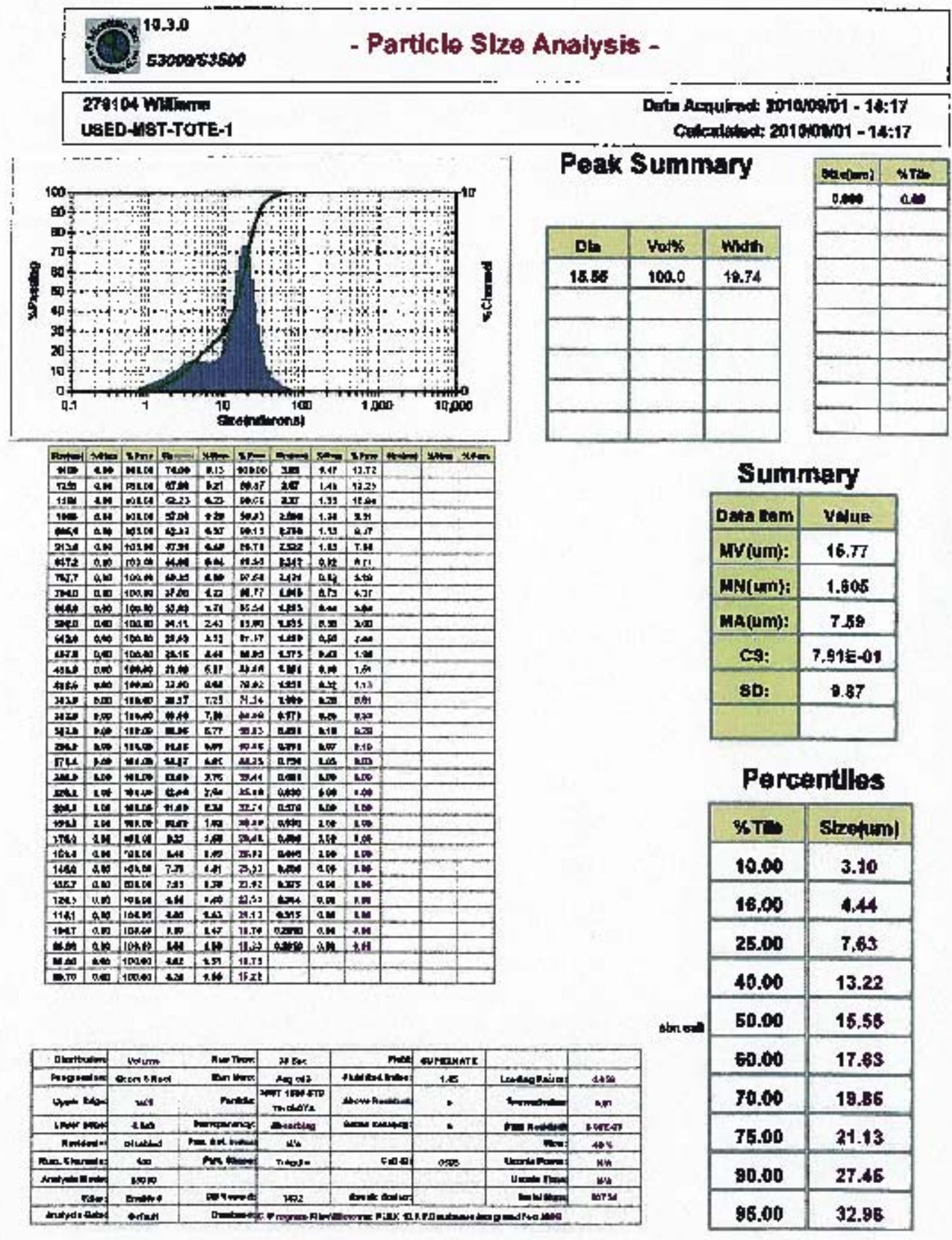




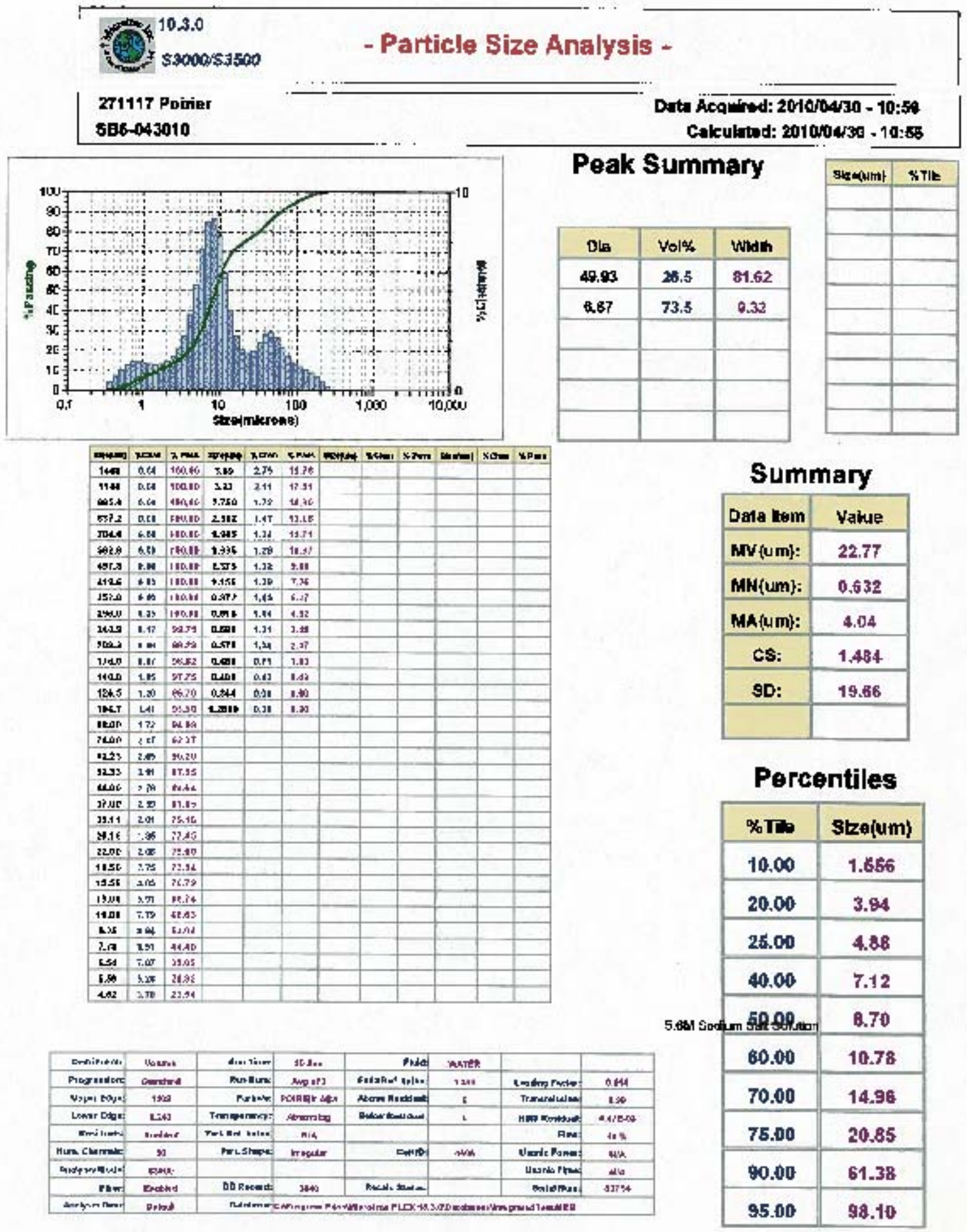


- Particle Size Analysis -

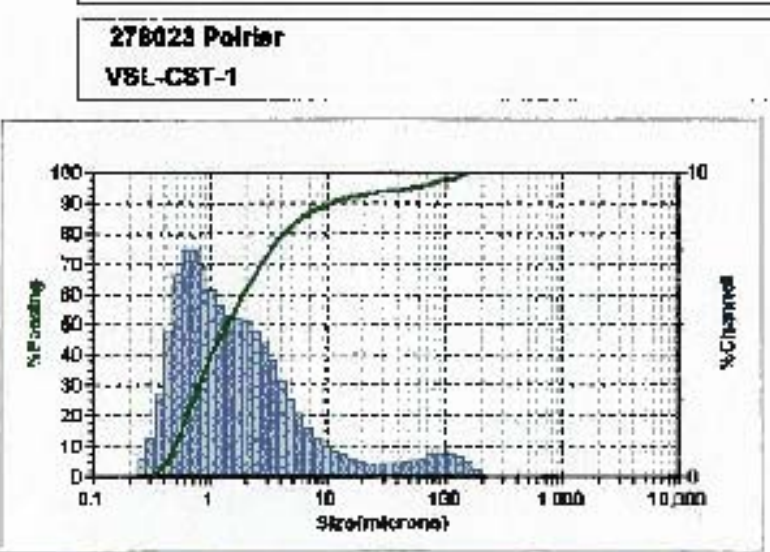

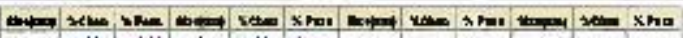

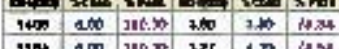

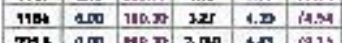

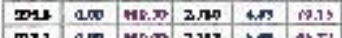

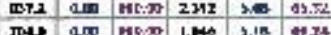

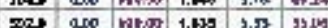

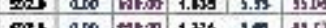

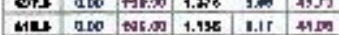

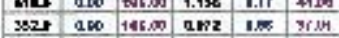

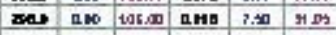

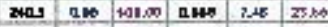

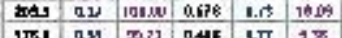

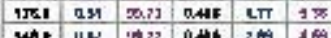

Nar UW

125. 2.11 20.91 0.244 1.91 100

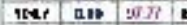

W.41 L4 20.9 ?

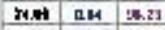

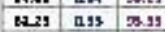

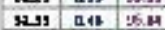

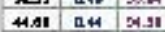

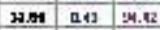

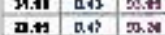

a.n 0.45 m..3

am $0.93 \quad x . m$

1153 dis

11.79 a.9

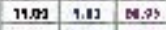

$2.201 .21 \quad 0.25$

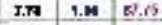

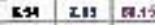

5.7 In MADO

240

(
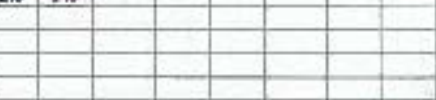
Data Acquired: 2010/10iv - 08:33 Cakulled: 20101019 - 08:33

Peak Summary
\begin{tabular}{|c|c|c|}
\hline DAB & Wol\% & WAdh \\
\hline 68.43 & 0.4 & 104.40 \\
\hline 1.219 & 91.6 & 3.00 \\
\hline & & \\
\hline & & \\
\hline & & \\
\hline & & \\
\hline
\end{tabular}

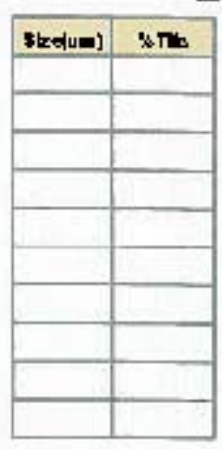

Summary
\begin{tabular}{|c|c|}
\hline Data tom & Valus \\
\hline MNium]: & 8.21 \\
\hline MN(um): & 0.5 \\
\hline MA(um): & 1.083 \\
\hline CS: & 5.54 \\
\hline SD: & 2.442 \\
\hline & \\
\hline
\end{tabular}

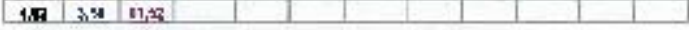

\section{Percentiles}

\begin{tabular}{|c|c|}
\hline$\%$ TIb & Sizei(um) \\
\hline 10.00 & 0.495 \\
\hline 20.00 & 0.833 \\
\hline 25.00 & 0.711 \\
\hline 40.00 & 1.029 \\
\hline 50.00 & 1.387 \\
\hline 50.00 & 1.930 \\
\hline 70.00 & 2.736 \\
\hline 75.00 & 3.34 \\
\hline 90.00 & 10.90 \\
\hline 95.00 & 51.62 \\
\hline
\end{tabular}

